Check for updates

Cite this: RSC Adv., 2021, 11, 2253

\title{
Organic dots (O-dots) for theranostic applications: preparation and surface engineering
}

\author{
Amin Shiralizadeh Dezfuli, (D) *ah Elmira Kohan, ${ }^{\text {b }}$ Sepand Tehrani Fateh, ${ }^{c}$ \\ Neda Alimirzaei, ${ }^{d}$ Hamidreza Arzaghi ${ }^{\text {(D) }}{ }^{\text {e }}$ and Michael R. Hamblin (D) fg
}

Organic dots is a term used to represent materials including graphene quantum dots and carbon quantum dots because they rely on the presence of other atoms $(\mathrm{O}, \mathrm{H}$, and $\mathrm{N})$ for their photoluminescence or fluorescence properties. They generally have a small size (as low as $2.5 \mathrm{~nm}$ ), and show good photostability under prolonged irradiation. The excitation and emission wavelengths of $\mathrm{O}$-dots can be tailored according to their synthetic procedure, where although their quantum yield is quite low compared with organic dyes, this is partly compensated by their large absorption coefficients. A wide range of strategies have been used to modify the surface of O-dots for passivation, improving their solubility and biocompatibility, and allowing the attachment of targeting moieties and therapeutic cargos. Hybrid nanostructures based on O-dots have been used for theranostic applications, particularly for cancer imaging and therapy. This review covers the synthesis, physics, chemistry, and characterization of $\mathrm{O}$-dots. Their applications cover the prevention of protein fibril formation, and both controlled and targeted drug and gene delivery. Multifunctional therapeutic and imaging platforms have been reported, which combine four or more separate modalities, frequently including photothermal or photodynamic therapy and imaging and drug release.

Received 20th September 2020 Accepted 8th November 2020

DOI: $10.1039 / \mathrm{d} 0 \mathrm{ra0} 8041 \mathrm{a}$

rsc.li/rsc-advances producing a new drug/treatment requires significant effort. Accordingly, a system as multifunctional as possible for controlling or considering a set of parameters is necessary. ${ }^{\mathbf{1 4}}$

From a diagnostic point of view, the system should be capable of not only detecting the intended disease with high sensitivity, but also should distinguish and differentiate the underlying disease from other possible diseases with similar characteristics and lab test results, which is known as specificity. ${ }^{15}$

Cancer and neurological diseases are some examples of complex diseases that require specific and sensitive diagnostic methods as the first step, and efficient and accurate treatment in the next step. ${ }^{\mathbf{2} 16}$ Accordingly, theranostics can be a possible solution for these types of problems. For designing an efficient theranostic system, researchers have to collect enough knowledge about both physiopathological processes of the diseases and material sciences.

Theranostics is defined as a combination of imaging and treatment modalities within a single molecular targeted platform, which can be applied in the next generation of personalized medicine. ${ }^{17}$ In the diagnostic function, the role of a theranostic agent is to report the presence of a disease, its location and its extent, and to allow the monitoring of the response to the therapeutic agent. ${ }^{18}$ For instance, one application is to allow more accurate tumor resection via image-guided surgery, and to allow post-surgical appraisal of the success of removal. To improve the accuracy of surgery, intraoperative 
imaging of diseased tissue is helpful due to the fact that the extent of the tumor may have changed after the initial presurgical imaging and during the course of the resection. ${ }^{19-22}$ Also, to confirm the complete removal of diseased tissue, postsurgical imaging is also useful. The second role of theranostics is to deliver or release therapeutic agents precisely to the targeted location. The agents that are delivered can be chemotherapeutic drugs (including, cisplatin, doxorubicin, and paclitaxel), biologics (such as proteins and antibodies), nucleic acids for gene therapy (DNA, siRNA, and miRNA), nanotherapeutic agents, and even therapeutic cells. ${ }^{23-25}$ These agents can be fabricated to be responsive to certain stimuli. The generation of a reactive form of molecular oxygen (singlet oxygen, ${ }^{1} \mathrm{O}_{2}$ ) with the capability to destroy the surrounding cells, which is triggered by light, is known as photodynamic therapy (PDT). In contrast, photothermal therapy is based on the absorption of photons by a chromophore, which creates heat from optical energy to kill cancer cells. ${ }^{26,27}$ The third role is the molecular alteration of a cellular or metabolic process. ${ }^{18}$ If particular cell surface receptors are engaged by theranostic agents, metabolic or cellular pathways may be disrupted, which can produce a therapeutic effect. ${ }^{28}$

Theranostic approaches combine multiple techniques into a single inclusive nanoplatform, often incorporating molecular imaging function. ${ }^{29}$ Molecular imaging can be based on optical imaging (fluorescence/bioluminescence/Raman), computed tomography (CT), magnetic resonance imaging (MRI), singlephoton emission computed tomography (SPECT), ultrasound (US), and positron emission tomography (PET). ${ }^{30-32}$ This technique can be applied for surveying a wide range of biological samples, from cells and ex vivo tissue samples, to in vivo imaging of living organisms and small animals. Moreover, it is capable of covering a broad range of sizes of organisms, from sub micrometer-sized viruses and bacteria, to macroscopic living biological organisms ${ }^{33-35}$ (Fig. 1).

In theranostic approaches, nanomaterials are often used for drug delivery and cancer imaging due to the fact that they allow the synergistic combination of diagnosis and therapy in a single nanoplatform..$^{36,37}$

Among the various groups of nanomaterials, carbon nanomaterials have strong absorption in the infrared (IR) and near infrared (NIR) region, and thus can be used for photothermal therapy (PTT) of cancer. Some organic nanomaterials such as carbon nanotubes and quantum dots show fluorescence in the visible and infrared regions for fluorescence imaging. ${ }^{32,38}$ Additionally, other carbon nanomaterials can convert the energy from a laser into acoustic signals, which makes them promising agents for photoacoustic imaging (PAI). ${ }^{39,40}$ Lastly, the inherent Raman vibration signals from carbon nanomaterials can also offer a method to track their distribution and metabolism in vivo. ${ }^{\mathbf{4 1 , 4 2}}$

Among the carbon nanomaterials, carbon-based quantum dots (CBQDs) including graphene quantum dots (GQDs) and carbon quantum dots (CQDs) show beneficial properties of low toxicity, environmentally friendly nature, simple and cost effective synthetic routes, and comparable optical properties to conventional semiconductor quantum dots and organic dyes. $^{\mathbf{4 3 - 4 5}}$ Photoluminescent CBQDs are superior to other

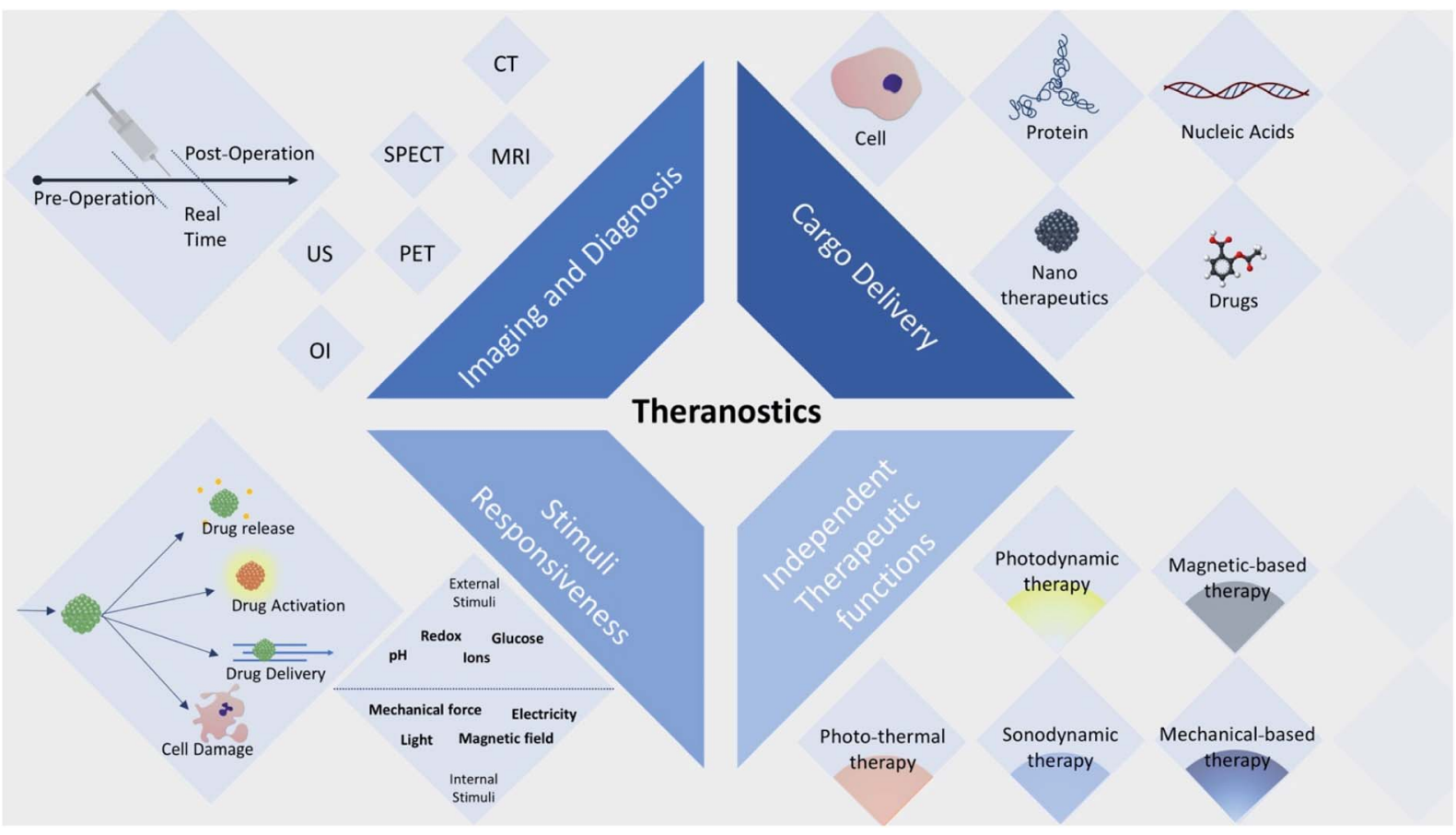

Fig. 1 Four aspects of theranostic agents: (1) cargo delivery (e.g. cells, proteins, nucleic acids, nanotherapeutics and drugs), (2) imaging via different imaging modalities (OI: optical imaging, US: ultrasound imaging, PET: positron emission tomography, SPECT: single-photon emission computed tomography, MRI: magnetic resonance imaging, and CT: computed tomography), (3) stimuli responsiveness to either external or internal or both stimuli and (4) independent therapeutic functions. 
quantum dots in terms of solubility, biocompatibility, resistance to photobleaching, chemical inertness, and suitability for biological applications (one-photon and multiphoton bioimaging, ${ }^{46,47}$ biosensors ${ }^{\mathbf{4 6 , 4 8 , 4 9}}$ and biomolecule or drug delivery $^{\mathbf{4 8 , 5 0 , 5 1}}$ ).

\section{Definition of organic dots (O-dots)}

In the last few years, CBQDs have been utilized in different biomedical applications, and many reports have discussed their synthesis, functionalization, combinations and applications. ${ }^{52-59}$ Although graphene quantum dots and carbon quantum dots have attracted significant attention, many researchers are still confused about the differences between these two sub-groups, and thus their correct and appropriate use in studies is challenging. GQDs are defined as graphene sheets with a size in the range of about 3-20 $\mathrm{nm}$, which possess photoluminescence properties due to their physiochemical characteristics mostly because of their size ${ }^{\mathbf{6 0}}$ while CQDs are photoluminescence spherical nanoparticles. ${ }^{61}$ CQDs are also referred to as carbon dots in some studies. However, there are some significant chemical, physical and optical differences between GQDs and CQDs, which will be discussed below.

As conventional in chemistry and biology, some materials can be tagged as organic materials, meaning that they are made of carbon, hydrogen and oxygen as the basic structure of the material, while they can be natural or synthetic. ${ }^{62}$ Accordingly, since GQDs and CQDs are materials with this composition of elements, we state that they can be categorized as organic materials and we intend to name them as "organic dots $(\mathrm{O}$ dots)" or luminescent organic clusters (LOC). This categorization may cause some implications including attracting attention to their chemical and biological properties, expansion of their biomedical applications and facilitating the design and fabrication of other types of quantum dot fluorescence materials. Moreover, we hypothesize that due to the emergence of synthetic biology, the biological synthesis of these materials can be expected (for example via enzymes), and thus this designation may provide new ways and approaches toward it. Herein, different aspects of O-dots are discussed.

A semiconductor quantum dot is a single electronic oscillator, whereas an O-dot is a clustered pack of isolated oscillators (phosphors). ${ }^{63}$ Depending on the reaction conditions (temperature, duration of the procedure, and the ratio of precursors), two types of O-dots can be produced. The first type is obtained under mild conditions, e.g. at a moderate temperature. Here, each dot is comprised of only one type of phosphor, assembled into a particle mainly due to weak (physical) forces. Upon further heating, type-I dots can be converted into type-II dots; however, this step is not reversible. Type II dots are obtained through deep carbonization of pristine organic substances, and are more like elemental carbon. In this structure, different oscillators are linked together via strong bonds (for instance, $\sigma$ bonds between carbon atoms). The absorption spectra of type-II O-dots do not show discrete bands because they consist of multiple independent oscillators (in contrast to type-I O-dots). Another distinguishing characteristic of type II O-dots is that their emission wavelength depends on the excitation wavelength. However in type-I O-dots, the excitation wavelength just affects the intensity of the luminescence, and not the wavelength (color) of the emitted light (Fig. 2). ${ }^{62}$

This review aims to summarize the recent advancements in the design and applications of O-dots in theranostics including bio-imaging, drug delivery, gene delivery and phototherapy. The organization and scope of this review are shown in Fig. 3.

\section{Chemical and physical properties of organic dots}

\subsection{Chemical properties}

The properties of O-dots including CQDs and GQDs depend mainly on their synthetic routes. Accordingly, the chemical

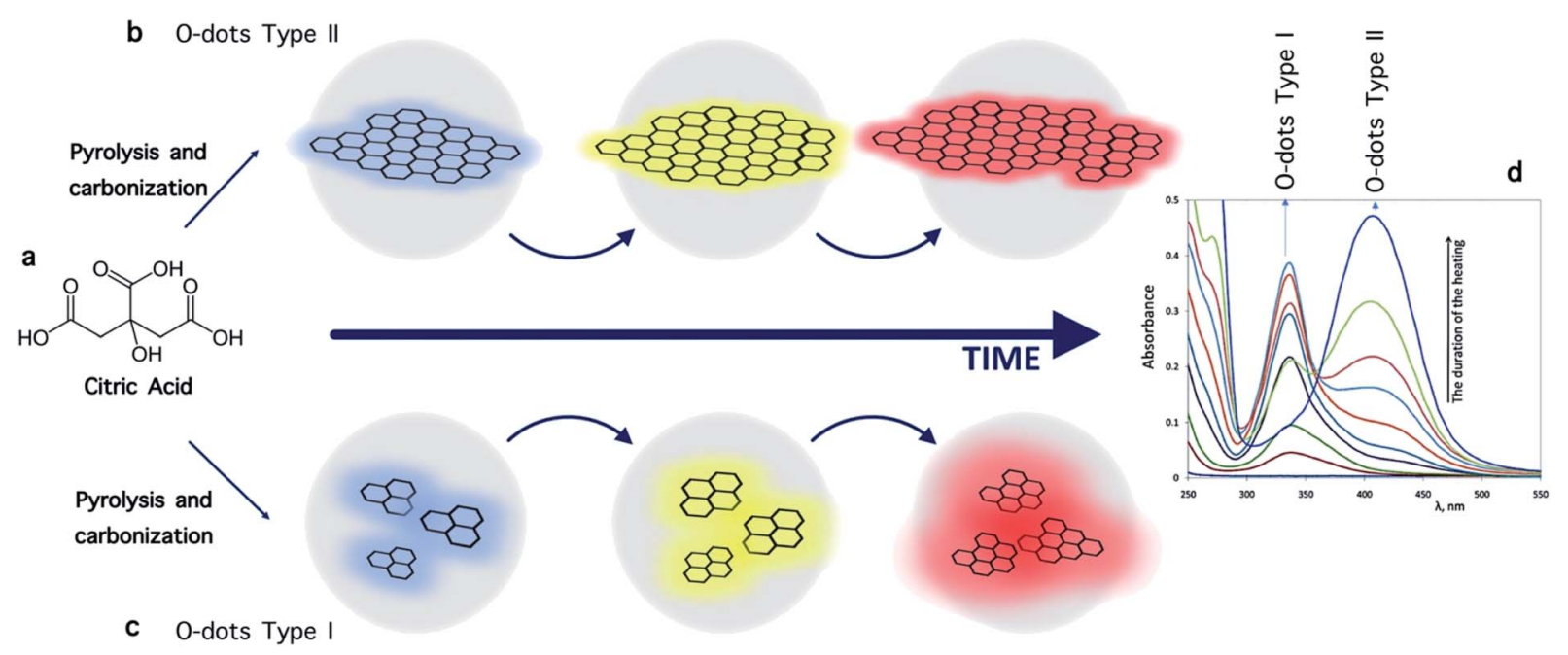

Fig. 2 (a) Formation of type I and type II carbon dots starting from citric acid as the carbon precursor. The intermediate "primary fluorophores" is a nominal unit. ${ }^{62}$ (b and c) Schematic illustration of the luminescence of O-dots upon excitation, which is related to their size. ${ }^{62}$ (d) Typical absorption spectra of O-dots. ${ }^{62}$ 


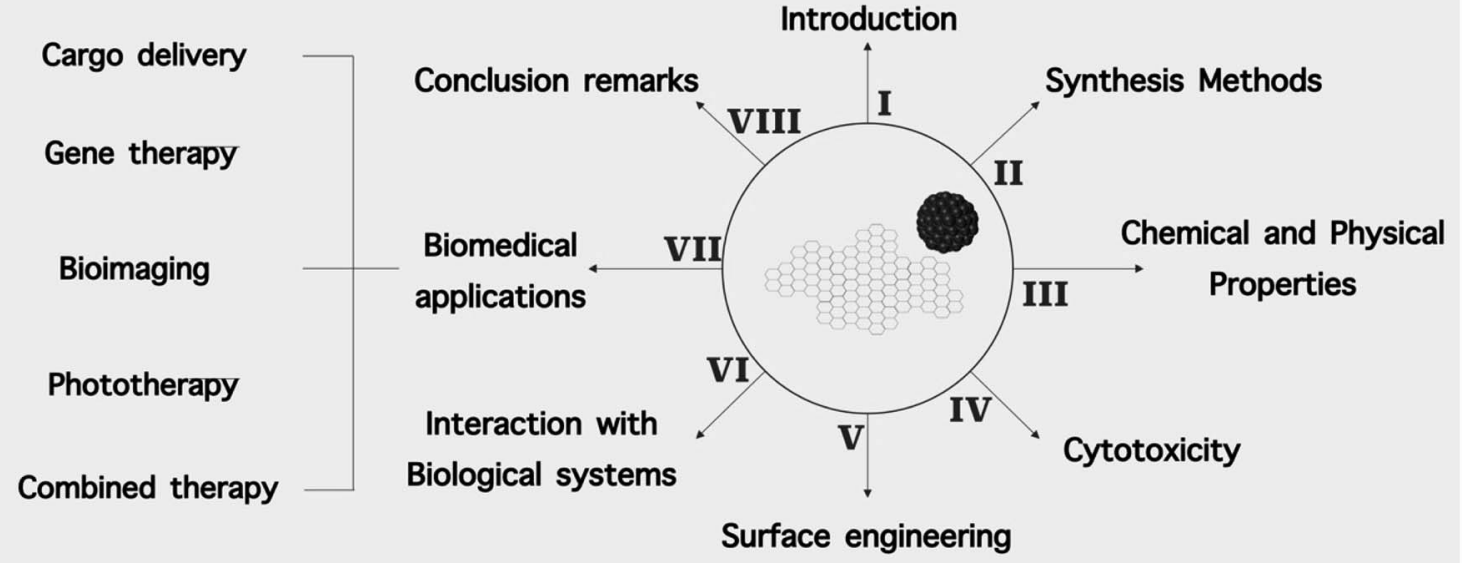

Fig. 3 Schematic illustration of the structure of this review.

structures of O-dots show large variability and chemical characterization is important to gain a better understanding of particular O-dots (Fig. 4).

For example, GQDs, are composed of a single or multiple graphene layers with chemical groups attached to the edges, and they are commonly anisotropic, with lateral dimensions much larger than their thickness. GQDs are synthesized from pristine few-layer-thick graphene flakes as a precursor. ${ }^{64}$ They have a narrow size distribution of $3-8 \mathrm{~nm}$ and small sheetshaped morphology, as shown by transmission electron microscopy (TEM) imaging. Due to the presence of a carbon core, GQDs have a crystalline structure with a lattice spacing of $0.24 \mathrm{~nm}$ comparable to the (100) facet of graphite and a honeycomb lattice with zigzag edges of $7 \mathrm{~nm}$ GQDs. This is in contrast
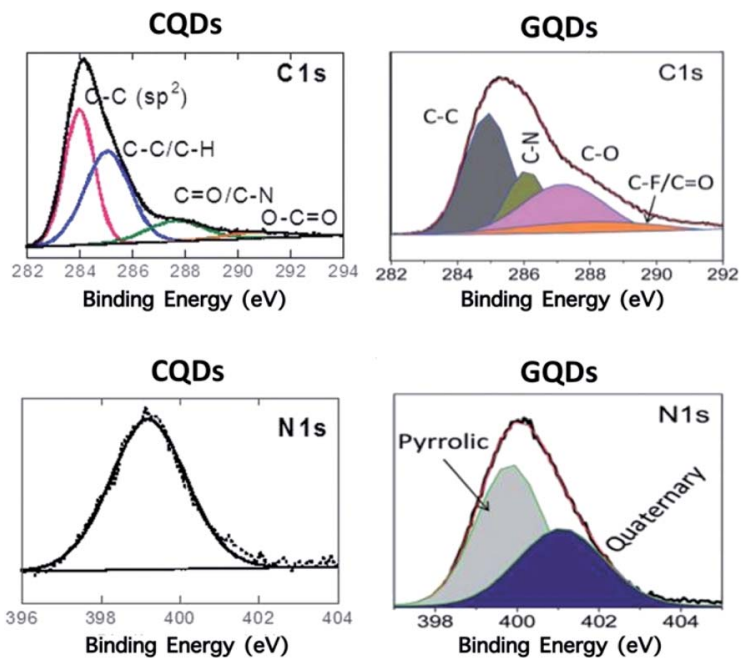

Fig. 4 Characteristics of organic dots. XPS bonds of CQDs (hydrothermal synthesis using 4-aminophenylboronic acid) ${ }^{66}$ (reproduced from ref. 66 with permission from the American Chemical Society, Copyright 2016). to the spherical CQDs that have been formed using ribonuclease A as a bimolecular templating agent under microwave irradiation, which have an interlayer distance of $0.34 \mathrm{~nm}$, matching the (002) facet of graphite. ${ }^{65}$

$\mathrm{X}$-ray diffraction (XRD) provides exact information about the crystalline structure of O-dots. The strong signal peak at $2 \theta=$ 26.51 shown in the XRD pattern of graphene flakes becomes weaker in GQDs, showing that GQDs are thinner than graphene flakes owing to the additional exfoliation of few-layered graphene. The broad peak positioned at $2 \theta \sim 21.91(d=0.41 \mathrm{~nm})$ may result from the p-p stacking of GQDs. No peaks are observed in the $2 \theta$ region of $5-20^{\circ}$, demonstrating that the GQDs are different from GO with fewer oxygen-containing groups. ${ }^{65}$ The XRD diffractogram of CQDs formed from 4-aminophenylbenzene displays a broad diffraction peak at around $21.73^{\circ}$ with an interlayer spacing of $0.42 \mathrm{~nm}$, which is larger than that of bulk graphite, indicating the poor crystallization and amorphous character of the structures. ${ }^{66}$

Using standard characterization methods such as X-ray photoelectron spectroscopy (XPS), Fourier transform infrared (FTIR) spectroscopy, and Raman spectroscopy, the different surface functionalities on O-dots can be characterized. The high-resolution C 1s XPS spectrum of CQDs prepared hydrothermally from 4-aminophenylboronic acid ${ }^{66}$ showed bands corresponding to $\mathrm{C}-\mathrm{C}(283.9 \mathrm{eV}), \mathrm{C}-\mathrm{C} / \mathrm{C}-\mathrm{H}(285.0 \mathrm{eV}), \mathrm{CQO} / \mathrm{C}-\mathrm{N}$ $(287.3 \mathrm{eV})$ and $\mathrm{O}-\mathrm{CQO}(290.3 \mathrm{eV})$. The band at $399.6 \mathrm{eV}$ in the $\mathrm{N}$ $1 \mathrm{~s}$ high-resolution XPS spectrum indicates the presence of amine groups. The C 1s XPS spectrum of GDQs formed by cutting pristine graphene flakes using an electrochemical redox reaction in an ionic liquid/water electrolyte under a reverse potential $^{65}$ showed peaks related to $\mathrm{C}-\mathrm{C} / \mathrm{C}-\mathrm{H}(285.0 \mathrm{eV}), \mathrm{C}-\mathrm{O}$ (286.1 eV, such as C-OH or C-O-C), C-N (287.2 eV), and O-CQO $(288.5 \mathrm{eV}$ ) (Fig. 4a). The N 1s band indicates the presence of two types of $\mathrm{N}$ atoms. The peak at $400.2 \mathrm{eV}$ can be assigned to the $\mathrm{N}$ atom of a pyrrolic structure (imidazolium group) adsorbed or located at the graphitic edge. Quaternary $\mathrm{N}(401.2 \mathrm{eV})$ atoms 
may be incorporated into the graphene layer and take the place of carbon atoms within the graphene plane. ${ }^{65}$

The chemical composition of O-dots can be obtained from Fourier-transform infrared spectroscopy (FTIR). The three strong bands at around 3425, 1720 and $1645 \mathrm{~cm}^{-1}$ shown in the FTIR spectrum of the GQDs synthesized by an electrochemical method are associated with the vibrations of the hydroxyl $(-\mathrm{OH})$, carbonyl (CQO) and graphitic (CQC) groups, respectively. The band at $1078 \mathrm{~cm}^{-1}$ is related to the alkoxy groups $(\mathrm{O}-\mathrm{C}-\mathrm{O})$ present in the GQDs. The spectrum reveals that GQDs have many oxygenated functional groups on their surface. Their FTIR spectrum is also significantly different from that of the precursor graphene flakes, with a weak adsorption band at around $3425 \mathrm{~cm}^{-1}$. The FTIR spectrum of CQDs depicts similar features of distinct strong bands at $3465 \mathrm{~cm}^{-1}$ (OH vibration) and $1618 \mathrm{~cm}^{-1}$ (CQO) with additional weak bands attributed to graphitic CQC $\left(1645 \mathrm{~cm}^{-1}\right), \mathrm{O}-\mathrm{C}-\mathrm{O}\left(1078 \mathrm{~cm}^{-1}\right)$ and $\mathrm{B}-\mathrm{O}$ $\left(1090 \mathrm{~cm}^{-1}\right)$, and stretching and deformation vibration modes of the boroxol bond of the boronic acid moieties. ${ }^{67}$ The presence of $\mathrm{C}-\mathrm{N}$ is demonstrated by the peak at $1400 \mathrm{~cm}^{-1}$.

Raman analysis of these organic quantum dots displays the carbon characteristic D and G bands at around 1350 and $1570 \mathrm{~cm}^{-1}$, respectively. The intensity of the $\mathrm{D}$ band, which is related to the presence of $\mathrm{sp}^{3}$ defects, and the $\mathrm{G}$ band, related to the in-plane vibration of $\mathrm{sp}^{2}$ carbon $\left(I_{\mathrm{G}} / I_{\mathrm{D}}\right)$, is a measure of the disorder in the nanostructure. The Raman spectrum of CQDs comprises a broad band at $2996 \mathrm{~cm}^{-1}$, corresponding to the $2 \mathrm{D}$ graphitic structures, with an $I_{\mathrm{D}} / I_{\mathrm{G}}$ ratio of $\approx 1.24$. In the case of graphene flakes used for the formation of GQDs, the weak D and strong $\mathrm{G}$ bands indicate the presence of slightly defective graphene flakes. The increase in the $I_{\mathrm{D}} / I_{\mathrm{G}}$ ratio $\left(I_{\mathrm{G}} / I_{\mathrm{D}}=0.6\right)$ of the formed GQDs suggests the formation of even more defective materials. ${ }^{68}$

\subsection{Optical properties}

O-dots normally show strong optical absorption in the UV region (260-320 nm) due to the $\pi-\pi^{*}$ transition of their $\mathrm{C}=\mathrm{C}$ bonds. The shoulder peak located at $270-390 \mathrm{~nm}$ is attributed to the $\mathrm{n}-\pi^{*}$ transition of the $\mathrm{C}=\mathrm{O}$ bonds. ${ }^{69}$ The location of the peak in the spectrum rather than the intensity is more affected by the preparation route. ${ }^{\mathbf{7 0 - 7 4}}$ Under UV irradiation, different emission colors have been observed for GQDs, which is related to the synthetic routes employed. For example GQDs can emit bright UV, ${ }^{75,76}$ blue, ${ }^{73,77}$ green,$^{78,79}$ yellow, ${ }^{\mathbf{8 0}, 81}$ red $^{79}$ and near infra-red $^{82}$ emissions. The PL emission in O-dots arises from quantum confinement effects, which can be excitationdependent and excitation-independent $\mathrm{PL}^{\mathbf{8 3 , 8 4}}$ and can be changed by altering the size of the quantum dots. ${ }^{68}$ The quantum yield (QY) is an important factor to evaluate the PL of O-dots. The QY of naked O-dots is very low, where GQDs have a higher QY compared to CQDs due to their special layered structure and crystallinity. However, despite the low QY commonly found in CQDs, some approaches have been investigated to improve their QY such as element doping, metalenhanced fluorescence $\mathrm{e}^{\mathbf{8 5 , 8 6}}$ and surface passivation/ modification. ${ }^{87}$

Non-blinking PL and exceptional photostability are the main benefits of O-dots compared to conventional organic or inorganic fluorophores. Under continuous excitation with an Xe lamp, the PL intensity of O-dots is hardly changed for several hours, while the emission of organic fluorophores is changed within minutes (photobleaching). ${ }^{88}$ Moreover, in the presence of solvents or biological systems such as serum, the PL of O-dots hardly shows any changes. ${ }^{88}$ Variations in the $\mathrm{pH}$ value may alter the photoluminescence of O-dots, particularly for $\mathrm{N}$-doped $\mathrm{O}$-dots. The change is more obvious when basic/acidic sites are involved in the PL emission of the O-dots. ${ }^{89}$ When the $\mathrm{pH}$ value is increased, the surface charge is converted from positive to negative. This phenomenon occurs because of the ionization of amine, carboxyl and hydroxyl groups. Due to the alterations in the $\mathrm{O}$-dots with a change in $\mathrm{pH}$ value, it is possible to create $\mathrm{pH}$ responsive nanomaterials based on O-dots. ${ }^{90}$ These O-dots will be capable of being triggered via $\mathrm{pH}$ changes for temporal- and spatial-controlled cargo release and bio-imaging. ${ }^{91}$

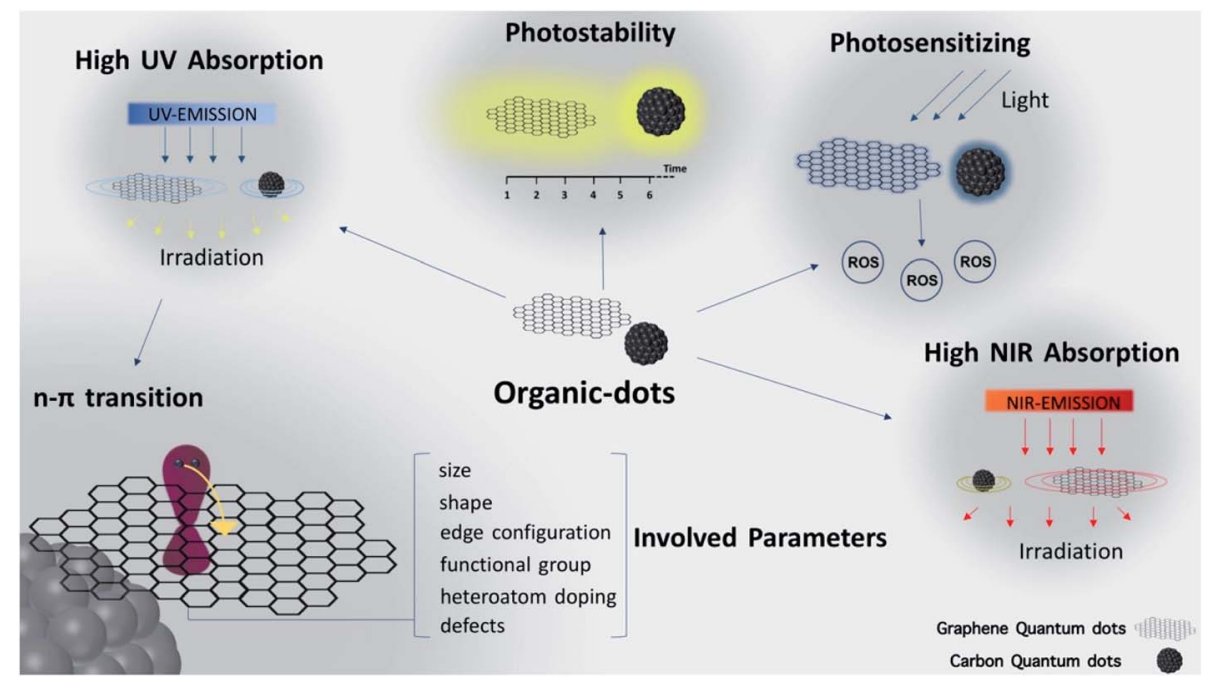

Fig. 5 Properties of organic dots. Both GQDs and CQDs possess various optical properties including high UV and NIR absorption, photosensitizing nature and high photo-stability. 
Although the precise mechanism of fluorescence in O-dots is not yet completely understood, two models have been proposed to account for the PL in CQDs. ${ }^{91}$ Based on density-functional theory (DFT) and time-dependent DFT, the PL of GQDs arises from the quantum confinement of the conjugated $\pi$ electrons in their $\mathrm{sp}^{2}$ carbon network, which can be governed by the size, edge configuration, shape, functional groups, heteroatom doping, and presence of defects. ${ }^{92}$ The PL process in O-dots mostly depends on their surface rather than the $\mathrm{sp}^{2}$ clusters inside their core. In fact, the recombination of electron-hole pairs creates PL in O-dots. ${ }^{93}$ This concept has been confirmed by introducing both electron donors and electron acceptors, which quench the PL of O-dots. ${ }^{87}$ Therefore, the optical properties of O-dots are governed by the interplay between the emissive sites and non-radiative trap sites on their surface, together with quantum confinement effects. ${ }^{94}$ CQDs have only a single excitation peak, which triggers the maximum emission, but GQDs regularly show two separate excitation peaks. The zigzag edges of GQDs are triplets like carbene, where they possess both $\sigma-\pi$ and $\pi-\pi^{*}$ transitions, which have an energy difference of $<1.5 \mathrm{eV}$ between the two peaks. ${ }^{80}$ The different aspects of the properties of O-dots are illustrated in Fig. 5.

\subsection{Effects of O-dots on cell viability and cytotoxicity}

When nanomaterials are used to deliver exogenous imaging reporters or therapeutic molecules in living organisms, their in vivo and in vitro toxicity must be evaluated. In vitro cytotoxicity testing involves a few different biochemical and morphological indicators, including cell proliferation, apoptosis, necrosis, oxidative stress, and DNA damage. These studies can provide sufficient information about the biocompatibility of foreign substances. The evaluation of in vivo toxicity depends on how foreign molecules or materials are exploited. The physiological response may vary to a large extent. Therefore, the investigation of the fate of an externally introduced material involves absorption, distribution, interaction, metabolism, retention, and excretion in a living organism. Another difficulty in the in vivo assessment is the possible toxic effects prompted by the interactions between the nanomaterial and the living organism. To investigate this toxicity, monitoring of body weight, blood chemistry panel, and hematology profile, and histological analysis are carried out. ${ }^{95,96}$ For carbon-based quantum dots (Odots), both their in vitro and in vivo toxicity have been widely studied, which are highly dependent on their shape, size, and surface coating ${ }^{96,97}$ (Fig. 6).

Zboril and co-workers carried out an inclusive in vitro cytotoxicity study on mouse fibroblasts (NIH/3T3) using 3 types of CQDs, which were differed in their surface functionalization, providing overall negative, positive and neutral charges. The results suggested that the neutral carbon quantum dots had low toxicity and higher safety up to concentrations of $300 \mathrm{mg} \mathrm{mL}^{-1}$. However, the negatively charged carbon quantum dots caused morphological changes in the cells, and stimulated proliferation with higher levels of oxidative stress by interrupting the G2/ $\mathrm{M}$ phase of the cell cycle; however, they did not enter the cell nucleus. In contrast, the positively charged CQDs showed the greatest toxicity to the cells due to the changes in the G0/G1 phase of the cell cycle, and they could also enter the cell nucleus, even at low concentrations. ${ }^{98}$

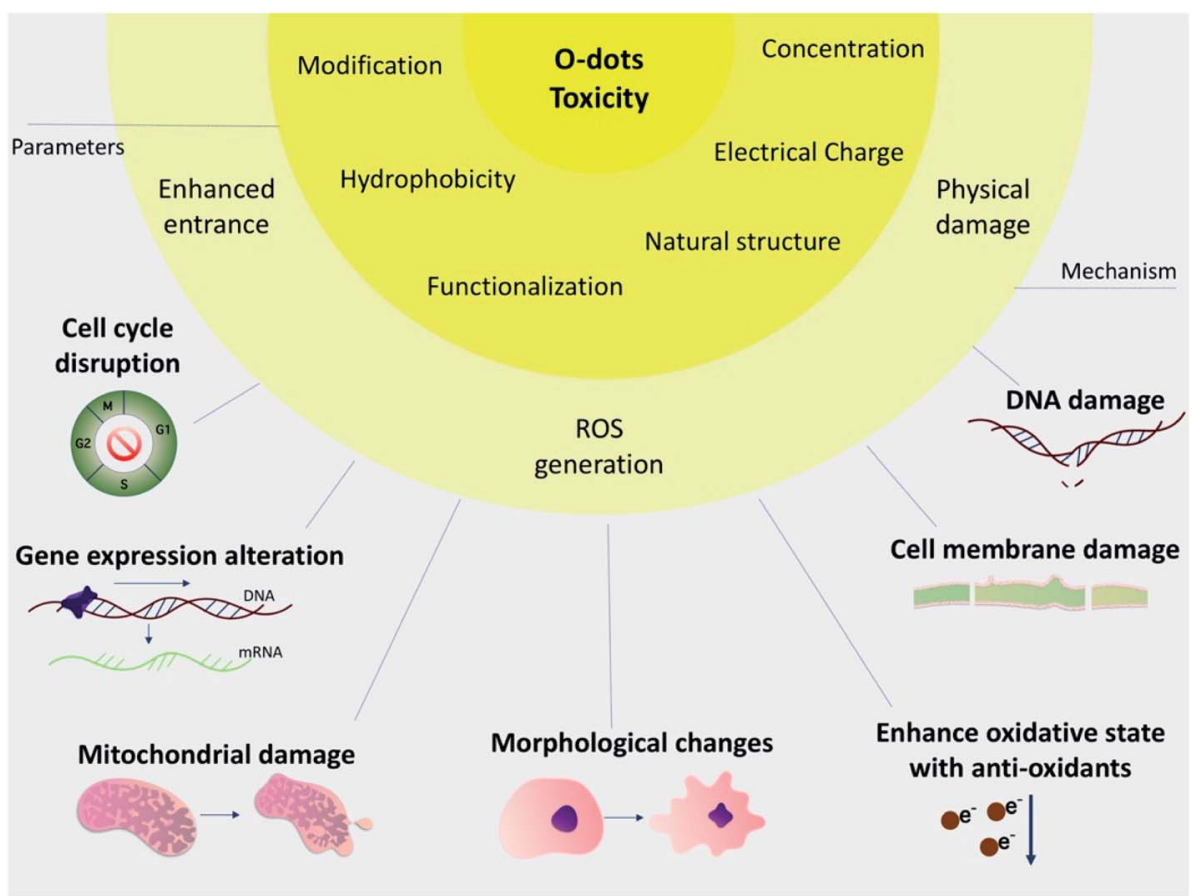

Fig. 6 Toxicity of O-dots. The parameters involved in the toxicity of O-dots are demonstrated above. These parameters define the mechanism of impact and together cause damage and alterations in biological systems. Some of these effects lead to cell death, while others may cause malfunctioning. 
a
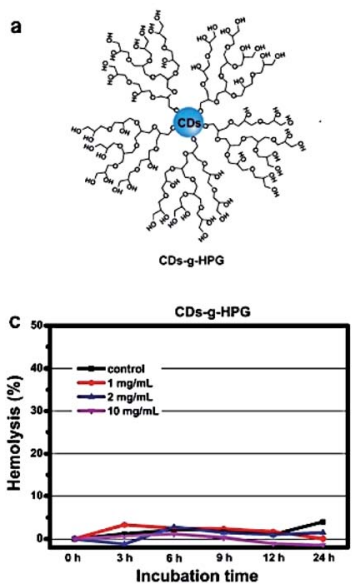
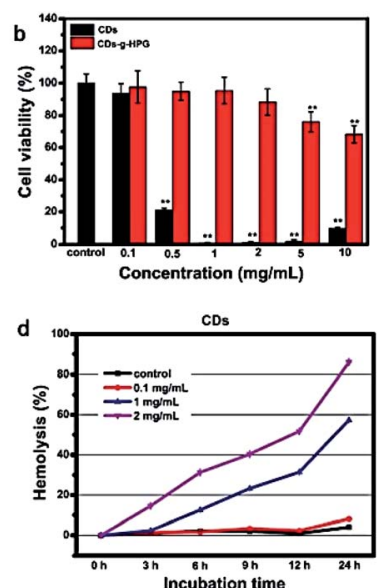

Fig. 7 (a) Structure of carbon quantum dots coated with HPG. (b) Cell viability of A549 cells after incubation with CDs or CDs-g-HPG at different concentrations for $24 \mathrm{~h}$ (c) and (d) lysis rate of RBCs incubated with CD-g-HPG and CD respectively ${ }^{101}$ (figure reproduced from S. Li, Z. Guo, R. Feng, Y. Zhang, W. Xue and Z. Liu, RSC Adv., 2017, 7, 4975, Published by The Royal Society of Chemistry).

The effect of surface charge on the cytotoxicity and uptake of CQDs produced from different ratios of citrate and spermidine as starting materials in human umbilical cord-derived mesenchymal stem cells was investigated by Yan et al. ${ }^{99}$ All the nanoparticles at concentrations below $50 \mathrm{mg} \mathrm{mL}^{-1}$ were nontoxic. The slightly positively charged CQDs showed a higher cell uptake efficiency than the negatively charged CQDs. In vivo assessments were recently done to examine the toxicity of CQDS derived from glucose, and their surface was stabilized with ethylenediamine using zebrafish as a model. ${ }^{\mathbf{1 0 0}}$
In another study, the toxicity of CQDs coated with hyperbranched polyglycerol (HPG) on red blood cells (RBC) was investigated (Fig. 7). Some CQDs show strong hydrophobic interaction with the RBC membrane, which can change the morphology of RBC and cause aggregation. Moreover, the rate of hemolysis with the use of these components was investigated. The cell viability was significantly lower than the control when treated with native CDs at a concentration as low as $0.5 \mathrm{mg} \mathrm{mL}^{-1}$. By contrast, the cell viability was not significantly different from the control when treated with CDs-g-HPG as high as $2 \mathrm{mg} \mathrm{mL}{ }^{-1}$. Therefore, conjugation with HPG can inhibit the hydrophobic interaction between the CQDs and RBCs, and therefore improve the biosafety of CQDs. ${ }^{\mathbf{1 0 1}}$

In the study by Wei et al., CQDs were synthesized via a calcination method using the plant material Gynostemma as a precursor, which required no toxic reagents or surface passivation chemicals. The toxicity of different concentrations of these CQDs (up to $400 \mu \mathrm{g} \mathrm{mL} \mathrm{m}^{-1}$ ) was tested in zebra fish, looking at embryonic development, and the nervous and circulatory systems. Due to the excellent fluorescence stability and biocompatibility of the CQDs, bio-imaging in zebra fish was successfully achieved, showing that the CQDs could enter the zebra fish embryos by the chorion or the mouth. Moreover, the anti-oxidant effect of the CQDs was investigated both in vitro and in vivo using oxidative stress induced by $\mathrm{H}_{2} \mathrm{O}_{2}$. Biomarkers were measured including, the level of reactive oxygen species (ROS) content and malondialdehyde (MDA). The oxidative stress markers were lower after treatment with CQDs compared to the control groups, showing that the fluorescent CQDs could reduce the oxidative damage by controlling the generation of ROS. Furthermore, the presence of the CQDs promoted the mRNA expression of related genes, which encode antioxidant
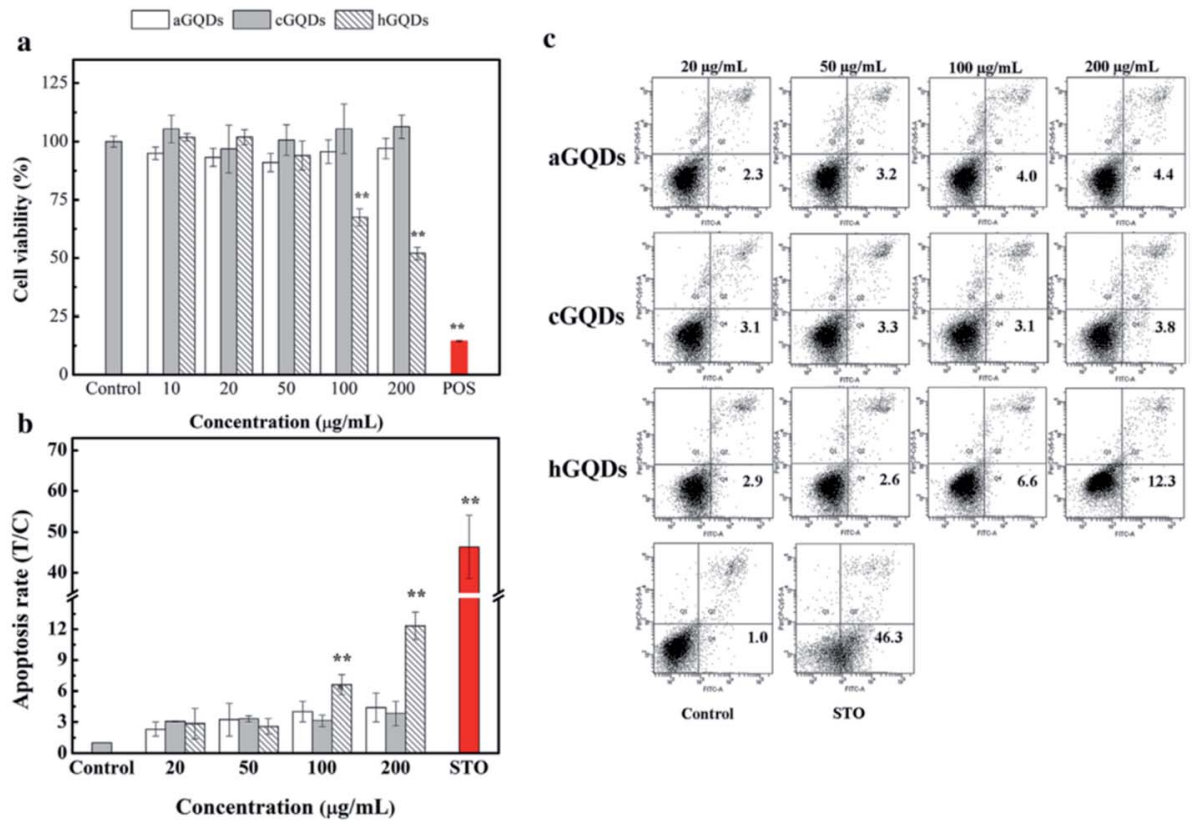

Fig. 8 Cytotoxicity assays of GQDs. (a) Cell viability results via WST-1 assay under $24 \mathrm{~h}$ of exposure with GQDs at different concentrations. (b) Rate of apoptosis in the cells treated with GQDs, obtained from flow cytometry with annexin-V-FITC/PI staining. (c) Quadrant analysis of the flow (figure has been reproduced from ref. 106 with permission from Elsevier, Copyright 2019). 
proteins to prevent oxidative damage in zebra fish. Therefore, fluorescent CQDs can be a potential candidate for the treatment of some diseases associated with oxidative damage. ${ }^{\mathbf{1 0 2}}$

Possible toxicity to mitochondria and metabolic disturbances are other important issues that must be addressed. The results from previous studies indicated that the mitochondrial toxicity of carbon quantum dots, which ultimately leads to cell death, was very low at concentrations of up to $500 \mu \mathrm{g} \mathrm{mL} \mathrm{L}^{-1}$ over a period of $24 \mathrm{~h}$, and the cell viability was higher than $82 \%$. Nevertheless, after increasing the concentration to $1 \mathrm{mg} \mathrm{mL}$ for longer than $24 \mathrm{~h}$, there a cell death rate of about $40 \%$ was observed. Thus, the dose-dependent toxicity of O-dots towards mitochondria can be used to deliver drugs to cells at low doses and kill cancer cells at high doses. ${ }^{\mathbf{1 0 3}}$

It is also important to assess the potential ability of GQDs to produce DNA damage since there is a close correlation between DNA damage and carcinogenesis. In a study reported by Wang et al., the genotoxicity of GQDs towards NIH- 3 T3 cells was investigated by flow cytometry analysis for DNA damage-related protein activation, while the GQD-induced ROS generation was studied as a potential explanation for DNA damage. The cellular uptake of GQDs and the cell death and proliferation of NIH-3T3 cells treated with GQDs were also studied to assess the cytotoxicity of GQDs. ${ }^{\mathbf{1 0 4}}$ An analysis of GQD-mediated photodynamic cytotoxicity was performed by Markovic et al., demonstrating that photodynamic activation could induce oxidative stress and generate in vitro cytotoxicity, and subsequent activation of both apoptosis and autophagy programmed cell death pathways. ${ }^{\mathbf{1 0 5}}$ However, studies on human breast cancer cells indicated that GQDs are non-toxic materials since they rapidly enter the cytoplasm but do not interfere with cell proliferation.

In another study using lung carcinoma A549 cells as a model, the cytotoxicity of three types of GQDs, including cGQDs (COOH-GQDs), hGQDs (OH-GQDs), and aGQDs ( $\mathrm{NH}_{2}$-GQDs) was investigated. The results showed that hGQDs were the most toxic since significant cell death was induced at a concentration of $100 \mu \mathrm{g} \mathrm{mL}{ }^{-1}$, as determined by the WST-1 assay as well as annexin-V-FITC/PI apoptosis analysis, whereas cGQDs and aGQDs were non-toxic in the tested concentration range (Fig. 8). ${ }^{106}$

The toxicity of GQDs to HeLa cells was tested using the CCK8 assay, showing that the cell viability decreased with an increase in GQD concentration. More than $90 \%$ cell viability was observed at concentrations ranging from 21.5 to $50 \mu \mathrm{g} \mathrm{mL}^{-1}$ and nearly $80 \%$ cell viability was obtained at the highest concentration of $200 \mu \mathrm{g} \mathrm{mL} \mathrm{mL}^{-1}$, which proves that a low concentration GQDs is biocompatible with low toxicity to HeLa cells. Furthermore, the lactate dehydrogenase (LDH) levels indicated the integrity of the cell membrane, confirming the low cytotoxicity of GQDs. ${ }^{107}$ In this work, the HeLa cells were exposed to different concentrations of GQDs for $24 \mathrm{~h}$ and then the $\mathrm{LDH}$ release was measured. The $\mathrm{LDH}$ release Hela cells incubated with various concentrations of GQDs for $24 \mathrm{~h}$ indicates that the $\mathrm{LDH}$ release levels were slightly higher than the control group at a low concentration of GQDs, suggesting that only a small fraction of the HeLa cell membrane was compromised by GQDs. However, compared to the control group, the
$\mathrm{LDH}$ release level increased to about $50 \%$ with a high concentration of GQDs, suggesting that GQDs could enter the cells through endocytosis ${ }^{\mathbf{1 0 8}}$ and cause corresponding membrane damage. The ROS assay is another effective technique to detect the oxidative stress levels in cells. Furthermore, the intracellular ROS level was low with GQDs at concentrations in the range of 0 to $50 \mu \mathrm{g} \mathrm{mL}{ }^{-1}$, while the ROS level significantly increased at a GQD concentration of $200 \mu \mathrm{g} \mathrm{mL}{ }^{-1}$. All these results suggest that a low concentration of GQDs displays relatively low cytotoxicity, while a high concentration $\left(200 \mu \mathrm{g} \mathrm{mL}{ }^{-1}\right)$ shows more cytotoxicity.

Chong et al. reported a detailed and systematic study on the in vivo toxicity of GQDs. ${ }^{109}$ To simulate the drug administration in humans, 48 female mice were randomly divided into four groups, including a control group and GQDs functionalized with polyethylene glycol (PEG-GQDs) administered intraperitoneally (i.p.), PEG-GQDs administered intravenously (i.v.) and PEG-GO administered intraperitoneally (i.p.). There was no obvious difference between the various administration routes of PEG-GQDs, and all the mice injected with PEG-GQDs survived compared with the control group (Fig. 9e). However, 3/12 of the
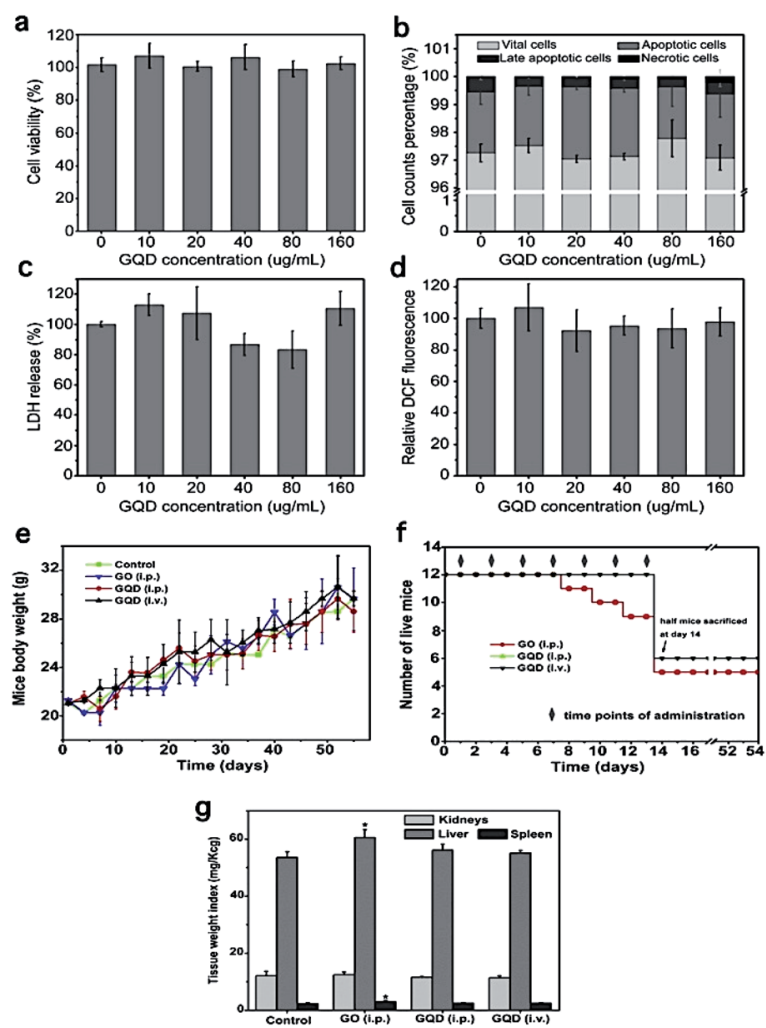

Fig. 9 Toxicity assays of graphene quantum dots. (a) WST-1 assay, (b) cell apoptosis and necrosis results, (c) LDH assay, (d) ROS generation assay, (e) weight of surviving mice with no difference compared with the control group, (f) effect of PEG-GQD and PEG-GO injection seven times into the mice and their living status, which is lower in GO. (g) Weight indexes of the main organs collected on day 40 from the mice injected with PEG-GO and PEG-GQDs, which indicates abnormality in the PEG-GO group. All these assays together demonstrate the low toxicity of GQDs (Figure has been reproduced from ref. 109 with permission from Elsevier, Copyright 2014). 
mice treated with PEG-GO died, while the remainder of the GO group displayed no significant loss in body weight (Fig. 9g). In addition, the weights (Fig. 9g) of the liver and spleen from the mice injected with PEG-GO were larger than that in the other groups, suggesting chronic damage to the liver and spleen caused by PEG-GO. In addition, dark spots were observed in the liver and spleen from the mice injected with PEG-GO, while nothing was found in the organs from mice injected with PEGGQDs. The other toxicity assay results are shown in Fig. 9. All the above results showed that the PEG-GQDs possessed lower cytotoxicity than PEG-GO due to the size-effect of graphene materials.

In a summary, it can be said that toxicity is a state of a biological system malfunctioning after the administration of materials to the body under certain conditions. The interaction of these materials with different components of biological systems is a reason for this malfunctioning, which is related to the characteristics of materials. In the case of O-dots, their electrical charge, functional groups, modification, morphology, hydrophobicity and concentration define the quality of the interaction between them and biological systems. Carbonbased materials are active materials, and this property allows the possibility of their efficient functionalization and modification, but also allows them to take part in unwanted reactions with biological components. Thus, by manipulating these parameters, it will be possible to come up with a structure that possess a balance between maximum function and minimum toxicity. As it mentioned previously, biological systems are complex and their different parts react differently; therefore, more studies on the interaction of different levels of biological systems (organelles, cells, tissues and organs) of different types are necessary.

\section{Synthesis of O-dots}

A variety of methods have been used for the preparation of $\mathrm{O}$ dots (CQDs and GQDs) (Fig. 10). In this section, we summarize the different approaches for the production of O-dots. Regardless of the specific carbon nanostructure, the synthetic approaches can be categorized into two main categories, i.e. the top-down and bottom-up methods.

Top-down methods are based on the progressive break down of larger carbon structures (e.g., graphite powder, carbon rods, carbon nanotubes, carbon black and even candle soot) ${ }^{\mathbf{1 1 0}}$ by various methods including, laser ablation, ${ }^{\mathbf{1 1 1}}$ hydrothermal, ${ }^{\mathbf{1 1 2}}$ electrochemical oxidation ${ }^{\mathbf{1 1 3 , 1 1 4}}$ and arc discharge ${ }^{\mathbf{1 1 5}}$ (Fig. 8a). This method offers some advantages such as abundant raw materials for the fabrication of O-dots, large-scale production, and simple operation. The obtained O-dots possess a highly crystalline nature and good aqueous dispersibility. However, these nanomaterials commonly demonstrate a predominant $\mathrm{sp}^{2}$ hybridized carbon structure, and therefore often lack an efficient electron band gap to provide fluorescence. Therefore, the size and surface chemistry should be modified via oxidizing agents such as concentrated acids $\left(\mathrm{HNO}_{3}\right.$ and $\mathrm{H}_{2} \mathrm{SO}_{4} / \mathrm{HNO}_{3}$ mixture). In this process, the bulk carbon materials are reduced into smaller fragments, while the surface is altered with oxygencontaining groups. This two-step route has become wellestablished for the formation of GQDs. In the first step, graphite is converted into graphene oxide (GO) sheets using the Hummers' method, while the second step involves cutting the GO into GQDs using various methods. ${ }^{59}$

The bottom-up approach employs certain molecular precursors that can form O-dots after dehydration and carbonization procedures. In general, the best precursors

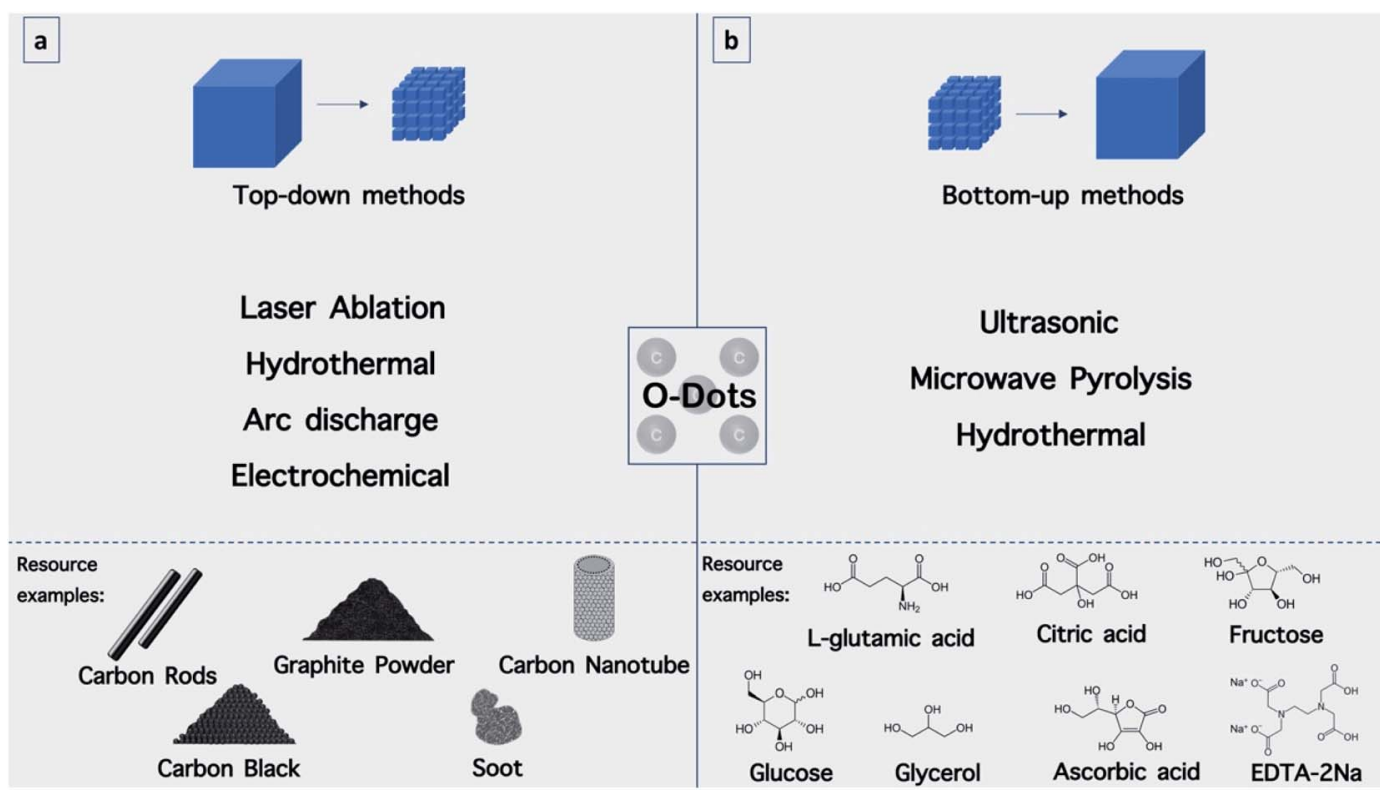

Fig. 10 Synthetic approaches for O-dots. (a) Top-down methods including laser ablation, hydrothermal, arc discharge and electrochemical methods with mentioned resources. (b) Bottom-top methods including ultrasonic, microwave pyrolysis and hydrothermal methods with the mentioned carbon precursors. 
possess $-\mathrm{OH},-\mathrm{COOH},-\mathrm{CQO}$, and $\mathrm{NH}_{2}$ groups, which can be dehydrated at higher temperatures. There are numerous approaches to perform the dehydration and carbonization processes, including hydrothermal, ${ }^{66}$ microwave-hydrothermal, ${ }^{116}$ plasma-hydrothermal approaches ${ }^{117}$ (Fig. 10b). These methods offer exciting opportunities to control the molecular size, and shape and fine-tune the physicochemical properties of O-dots.

\subsection{Top-down approaches}

The main idea of top-down methods is based on the fabrication of nanostructures from bulk materials. As will be discussed further in detail, in the top-down methods, bulk materials are broken down into their primary building blocks by applying an external source of energy and then become reconstructed into nanostructures with certain morphologies and atom configurations (Fig. 10a). An external source of energy, bulk carbon precursors and a suitable medium are the requirements for these methods, and based on these parameters, it is possible to invent new top-down fabrication methods (Fig. 11).

4.1.1. Electrochemical synthesis. Electrochemical etching of various carbon-based electrodes is a practicable low-cost procedure for the formation of O-dots (Fig. 11a). In this method, carbon electrodes such as graphite-rod electrodes are electrochemically broken down into CQDs and GQDs. One of the first electrochemical syntheses of GQDs was reported by Zhou et al., ${ }^{118}$ in which GQDs were prepared during electrochemical cycling of MWCNTs as carbon precursors in the presence of tetra- $n$-butyl ammonium ions (Fig. 12). The oxidation of graphitic electrodes at $+3.0 \mathrm{~V}$ was proposed by Zhao et $a .^{119}$ to prepare CQDs with emission wavelengths at $445 \mathrm{~nm}$ and $510 \mathrm{~nm}$.

Alkali-assisted electrochemical etching allowed the preparation of CQDs with a controlled size. ${ }^{\mathbf{1 2 0}}$ The electrochemical synthesis of photoluminescent CQDs from glycine under alkaline circumstances was recently reported by Wang et al. ${ }^{\mathbf{1 1 4}}$ The application of $+10 \mathrm{~V}$ between two Pt electrodes led to the oxidation of glycine (Fig. 9a) and resulted in the formation of ammonium ions, which further reacted with non-oxidized glycine through an amidation reaction. The ions produced allowed electro-polymerization, carbonization and passivation to form highly fluorescent CQDs with an average size of $2.4 \pm$ $0.4 \mathrm{~nm}$ and a crystalline structure.

In another study, water-soluble red fluorescent GQDs with a uniform size of $3 \mathrm{~nm}$ and red emission were successfully prepared without any chemical modification via the electrochemical exfoliation of graphite in a $\mathrm{K}_{2} \mathrm{~S}_{2} \mathrm{O}_{8}$ solution. The RFGQDs were isolated $\mathrm{sp}^{2}$ domains with a diameter of $3 \mathrm{~nm}$ generated by the very active $\mathrm{SO}_{4}{ }^{-}$radicals produced from $\mathrm{S}_{2} \mathrm{O}_{8}{ }^{2-}$ as electrochemical "scissors" to precisely cut the graphene sheets into small intact $\mathrm{sp}^{2}$ structures. ${ }^{70}$

4.1.2. Arc discharge method. Arc discharge is the electrical breakdown of a gas to form plasma, which has been widely applied for the preparation of carbon nanomaterials. ${ }^{\mathbf{1 2 1}}$ The chamber of this system contains two electrodes, i.e. the anode, which contains powdered carbon precursors, and the cathode, which is frequently a pure graphite rod. After filling the chamber with a gas or a liquid and applying a power supply (AC or DC), the electrodes are brought into contact to generate an arc. During arc discharge, a large amount of heat is produced, resulting in sublimation of the carbon precursors in the anode, which then move towards the cathode where they are deposited in the form of nanostructures (Fig. 11c). After oxidation of the crude materials (soot) in nitric acid, alkaline extraction and purification via gel electrophoresis, hydrophilic fluorescent CQDs are formed. ${ }^{122}$ The main weakness of this approach is the existence of different sized and non-uniform carbon nanostructures in the final mixture, which are often hard to separate.

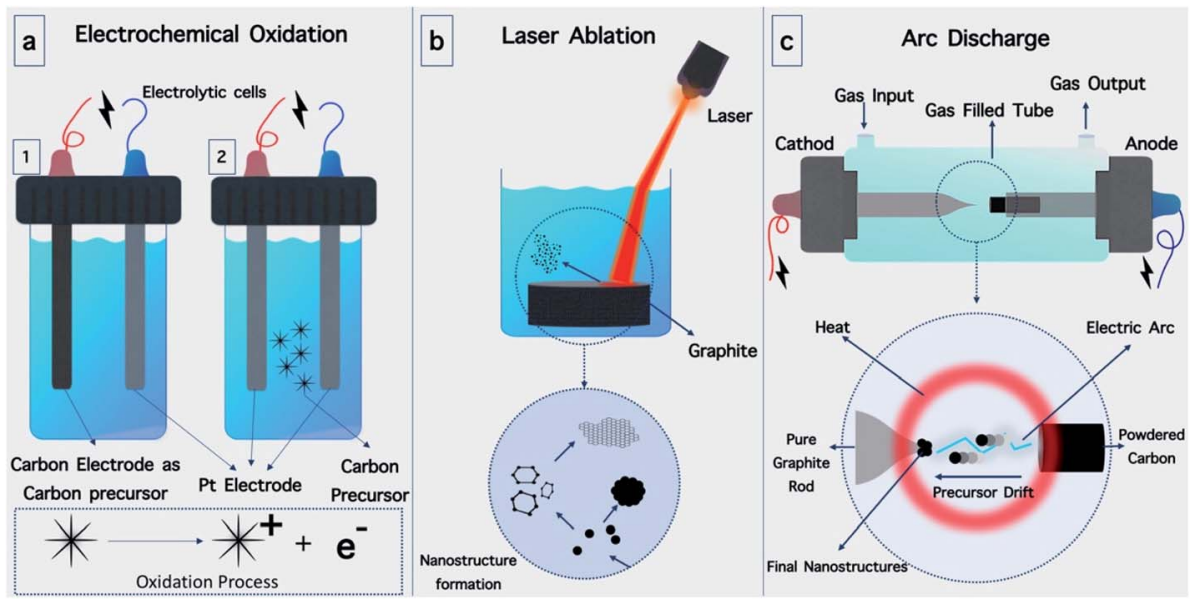

Fig. 11 Major top-down synthetic methods. (a) Electrochemical oxidation is performed in electrolytic cells, which can be divided into two types. (1) One electrode is made of carbon, while the other one is platinum. The carbon electrode in this electrolytic cell acts as a carbon precursor. (2) In the second type of cell, both electrodes are made of platinum, and the carbon precursors are dispersed in the medium. (b) Laser ablation method. Laser irradiation is used as a source of energy to break down graphite into carbon atoms and form GQDs or CQDs in the medium. (c) Arc discharge between two electrodes in a gas-filled tube cut out of carbon atoms from powdered carbon. Carbon atoms drift into the cathode forming carbon structures. 


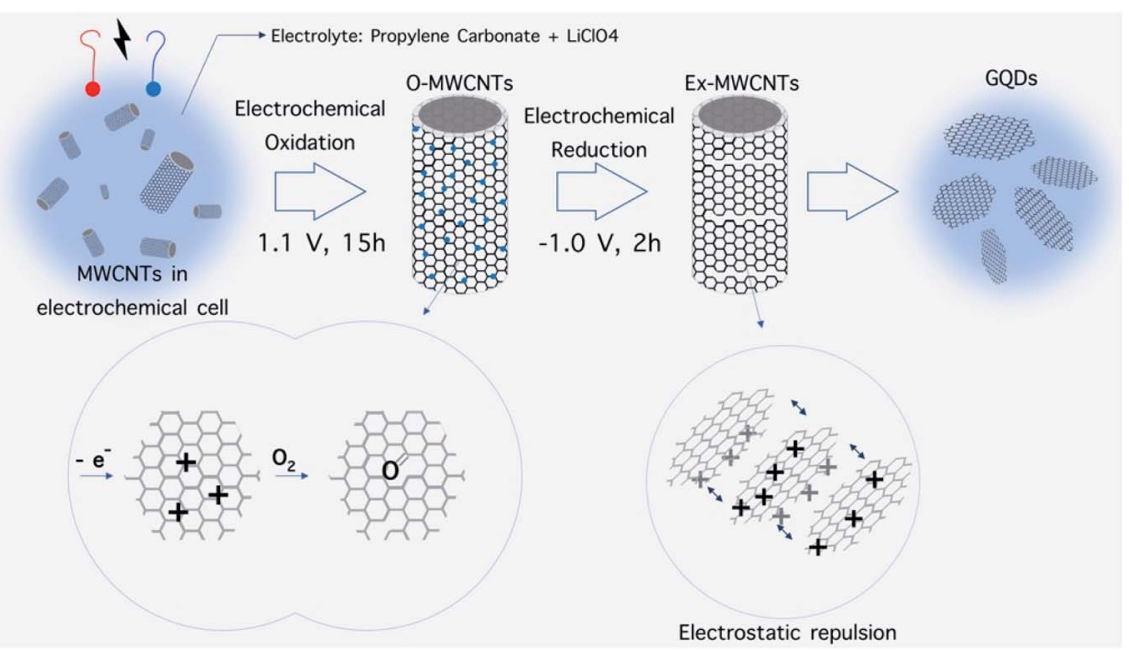

Fig. 12 Electrochemical preparation of GQD from MWCNT. Electrochemical oxidation of MWCNTs and subsequent electrochemical reduction leads to break down of sheets and electrostatic repulsion, respectively. ${ }^{118}$

4.1.3. Laser ablation. A rapid way to synthesize CQDs is the laser ablation of carbon-based bulk materials (Fig. 11b). Although, there are several interesting reports using laser ablation for the synthesis of CQDs, the large-scale synthesis of CQDs via laser ablation is rather difficult due to the narrow zone of target erosion and the low synthetic yields. Surface passivation can be achieved by further refluxing in $\mathrm{HNO}_{3}$ for $12 \mathrm{~h}$ and post-modification with polyethylene glycol (PEG) groups. The laser ablation of activated carbon ( $4 \%$ ash) in a water/ethanol solution (5:2) was reported by Yogesh et al., which led to CQDs $4 \mathrm{~nm}$ in size. ${ }^{123}$ A one-step process based on the irradiation of a graphite target using a $532 \mathrm{~nm}$ second harmonic beam of an Nd:YAG laser in $\mathrm{H}_{2} \mathrm{O}$ /ethanol or diethylenetriamine penta acetic acid solution for the formation of $3 \mathrm{~nm}$-sized CQDs was described by Tarasenka et al. ${ }^{\mathbf{1 2 4}}$ The synthesized particles exhibited strong photoluminescence in the visible region. The laser ablation of a solid carbon material in a liquid environment with laser pulses of 1064, 532 and $355 \mathrm{~nm}$ at different irradiation times was recently investigated. ${ }^{111} \mathrm{~A}$ wide size distribution of the CQDs was observed for the $1064 \mathrm{~nm}$ laser due to the deeper penetration of the laser into the dielectric during the ablation process, and therefore this wavelength is less suitable for the formation of size-controlled CQDs.

\subsection{Bottom-up approaches}

Since GQDs and CQDs are formed from carbon atoms organized in specific configurations, some carbon-based precursors can be used for the synthesis of these nanomaterials (Fig. 10b). Accordingly, these precursors act as carbon backbones, which can join together in a specific manner to form GQDs or CQDs under specific synthetic condition. For this purpose, a carbon precursor, a source of energy and a suitable medium are required. Specifically, the building blocks of GQDs and CQDs in the bottom-top methods are materials with small carbon chains that can merge under irradiation of an energy source and form honeycomb sheets of carbons or spherical carbon nanoparticles. The synthetic conditions can explain the difference between the formation of GQDs and CQDs since different bonds will be formed under different conditions.

4.2.1. Pyrolysis or carbonization of organic precursors. The pyrolysis of small organic molecules involves three steps of condensation, nucleation, and subsequent formation of larger O-dots. These steps are carried out by heating small organic molecules above their melting point. The precursors of this method can be organic salts (e.g., octadecyl ammonium citrate or diethylene glycol ammonium citrate $^{\mathbf{1 2 5}}$ ), coffee grounds, ${ }^{126}$ glycerol, ${ }^{127}$ L-glutamic acid, ${ }^{128}$ ascorbic acid, ${ }^{90}$ citric acid, ${ }^{129,130}$ and ethylenediaminetetraacetic acid disodium salt (EDTA$2 \mathrm{Na}){ }^{131}$ Besides simple combustion, plasma ${ }^{132,133}$ and microwave $^{\mathbf{1 3 4}}$ heating methods have been also explored. These methods are simple, economical, accessible, and allow the natural inclusion of heteroatoms, which are derived from the precursors. ${ }^{135}$ John and coworkers utilized a simple and costeffective technique in which the pyrolysis of cotton was applied as a green method for the preparation of CQDs. Low cytotoxicity, good photostability and broad blue and green fluorescence emissions were the advantages of the CQDS produced by this procedure. ${ }^{\mathbf{8}}$

4.2.2. Microwave-assisted synthesis. Microwave-assisted synthesis is an effective and time-saving approach for synthesizing O-dots. The in situ and transient heating procedure, increased production yield and improved chemical and physical properties are some of advantages of this method. ${ }^{136,137}$ The microwave pyrolysis approach was described by Zhu et al. for the first time. ${ }^{138}$ In their work, CQDs were produced by mixing PEG200 with a saccharide component such as glucose or fructose in distilled water, in a $500 \mathrm{~W}$ microwave oven for 2-10 min. Subsequently, the color of the solution changed from colorless to dark brown, showing the formation of CQDs. In another approach, CQDs were synthesized using poly(ethylenimine) (PEI) and glutaraldehyde as precursors via a microwave-assisted green synthetic procedure. Changing the molar ratio of glutaraldehyde to PEI led to the formation of different emission colors of CQDs, ranging from blue to yellow. ${ }^{139}$ 


\section{Surface engineering of $\mathrm{O}$-dots}

For better interacting with biological systems, modification of O-dots with different molecules and structures including biomolecules is necessary. Moreover, surface modification can alter the surface characteristics of a material to make it more suitable for a particular application. ${ }^{\mathbf{1 4 0 , 1 4 1}}$ For this purpose, the addition of functional groups on O-dots will facilitate this process. Also, functionalization may change some of their physical and chemical characteristics. ${ }^{\mathbf{1 4 2}}$

The surface functional groups available on the surface of $\mathrm{O}$ dots depend on the type of precursors and the reaction conditions. ${ }^{59}$ The functional groups present on the surface of O-dots include $-\mathrm{OH}$ and $-\mathrm{COOH}$ depending on the degree of oxidation. These groups readily form hydrogen bonds with water molecules, and their presence leads to reasonable solubility in water. Furthermore, these groups play a vital role in the enhancement of the PL efficiency. Therefore, alterations in the degree of oxidation can affect the optical properties of the O-dots. The QY of GQDs increases with a reduction in the oxygenation rate of GQDs, while the emission wavelength will be shifted towards longer wavelengths (red-shift) by their oxidation..$^{\mathbf{9 2 , 1 4 3 - 1 4 5}}$ In addition to these beneficial functional groups, additional functionalization with other materials such as polyethylene glycol (PEG) is necessary to improve the biocompatibility and also the QY of organic dots. The need for surface passivation offers some constraints in the synthetic procedure, which increases the overall particle size, resulting in a deleterious effects on the applications in of O-dots in different fields. PEG molecules, which are applied as surface passivation agents, can increase the inherent fluorescence emission. ${ }^{87,135}$ In addition to PEG, other small molecules such as ethylene diamine, octadecylamine, and 2-(2-aminoethoxy)-ethanol have been covalently linked to the surface of O-dots via an amide bond. Surface passivation leads to hydrophilicity and hydrophobicity in organic dots based on the nature of the functional groups. ${ }^{\mathbf{1 2 5 , 1 4 6}}$ Furthermore, the addition of heteroatoms (especially nitrogen) can improve the PL and QY of carbon dots. N-doping of GQDs increases their QY and produces a blue-shifted emission due to the strong electronwithdrawing ability of the $\mathrm{N}$ atoms within the conjugated $\mathrm{C}$ plane. ${ }^{80,92,130,147}$ To add catalytic functions to organic dots or to improve their PL properties, other elements (e.g., $\mathrm{Si}^{148} \mathrm{P}^{149} \mathrm{~S}^{150,151}$ and $\mathrm{B}^{\mathbf{1 0 8}}$ ) have also been doped into CQDs and GQDs. $\mathrm{S}$ and $\mathrm{N}$ codoped CQDs and GQDs can have a QY value as high as 73\% and $71 \%$, respectively. ${ }^{\mathbf{1 0 8 , 1 5 0}}$ Thus far, different functional groups such as amine, carboxyl, quaternary ammonium and alkoxysilane have been coated onto the surface of O-dots (Fig. 13). The most common groups detected on the O-dot surface are amine and carboxyl, which allow the conjugation of organic, polymeric, inorganic or biological moieties..$^{152-154}$ Herein, some approaches for surface modification will be discussed.

\subsection{Amine capped CDs}

Branched poly(ethylenimine) (BPEI), 2,2'-(ethylene-dioxy)bis (ethylamine) (EDBEA), 4,7,10-trioxa-1,13-tridecanediamine (TTDDA), poly(ethylene glycol)diamine (PEG1500N), ethylenediamine (EDA), polyenepolyamine (PEPA), tetraethylenepentamine (TEPA), urea and chitosan have been used to coat the surface of O-dots due to their amine groups. The synthesis of these types of coated O-dots is carried out using different approaches including microwave irradiation, hydrothermal carbonization, and pyrolysis, which convert carboxyl groups to amine groups.

5.1.1. Microwave irradiation. This simple and rapid approach has been utilized for the preparation of amine-capped CDs. Citric acid (CA) and ethylenediamine (EDA) are customarily used to fabricate the carbon core with amine groups on the surface. A colorless solution of CA and EDA is combined under vigorous stirring. Next, the clear transparent solution is placed in a microwave oven and heated for a certain time. ${ }^{155}$ After dissolution, sonication and dialysis, moderately pure amino-

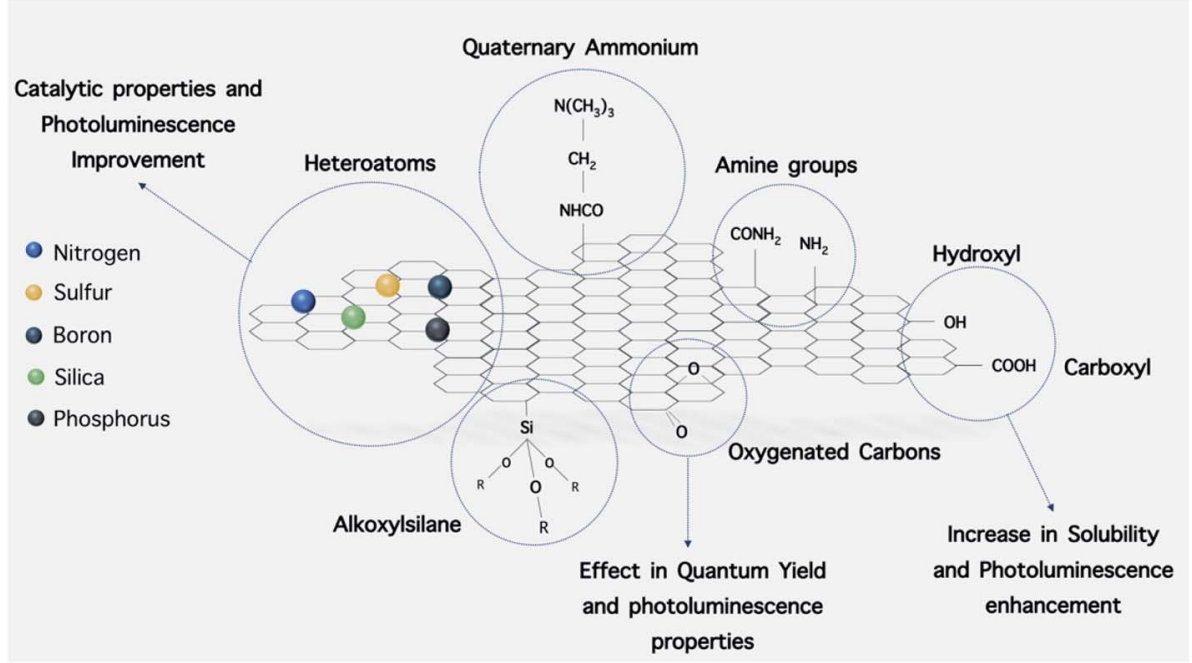

Fig. 13 GQD functionalization and modification with other elements. Functional groups and heteroatoms add different properties to GQDs (i.e. increase in solubility, better photoluminescence and catalytic properties). 
capped CQDs are produced. ${ }^{153,154,156}$ Another material used for the preparation of amine-capped CQDs is 2,2'-(ethylenedioxy) bis(ethylamine) EDBEA. When the color of the transparent solution changes from a colorless liquid to a brown solid precipitate, the amine-coated C-dots are ready to be collected. ${ }^{157,158}$ Multicolor carbon dots produced from D-fructose and $\mathrm{NH}_{4} \mathrm{HCO}_{3}$, called F-CQDs, were synthesized via a time and energy-saving microwave-assisted approach. Manganese oxide carbon quantum dots were synthesized via a microwave approach. These materials were used for bioimaging and cell tracking via fluorescence microscopy for up to 12 cell generations with only nominal cytotoxicity. ${ }^{88}$

5.1.2. Hydrothermal method. The hydrothermal approach is an eco-friendly method and has attracted significant attention. CQDs were produced by mixing citric acid and EDA at $200{ }^{\circ} \mathrm{C}$ for $5 \mathrm{~h}$. As a result of citric acid and EDA condensation, polymer-like CQDs were formed. Subsequently, the polymer was carbonized to yield amino-coated CQDs. ${ }^{\mathbf{4 6 , 1 5 9 , 1 6 0}}$ Chitosan is another material used to synthesize amine-capped O-dots due to its abundant amino/oxygen groups. ${ }^{\mathbf{1 6 1}}$ The hydrothermal carbonization of chitosan was carried out in a $2 \%$ aqueous solution of acetic acid in a Teflon-lined stainless steel autoclave at $180{ }^{\circ} \mathrm{C}$ for $12 \mathrm{~h}$. After centrifugation and removing the black precipitate, amine-capped O-dots were obtained. ${ }^{162,163}$ Mintz and his team reported the preparation of tryptophan quantum dots, which could easily cross the blood-brain barrier via the LAT1 transporter, which made them an appropriate candidate for drug delivery and imaging of brain tissue. Two other nitrogen dopants, i.e. urea and 1,2-ethylenediamine, were also used in this approach. The carbon dots possessed an excitation wavelength-dependent emission, low toxicity, and were observed inside the central nervous system of zebra fish (Danio rerio). This observation confirmed that tryptophan quantum dots can cross the blood-brain barrier. ${ }^{88}$

5.1.3. Pyrolysis. In this method, materials such as polyenepolyamine (PEPA) are used as a primary amine source to prepare O-dots coated with amine groups. ${ }^{152}$ Using this approach, a mixture of citric acid and PEPA was heated to $170^{\circ} \mathrm{C}$ in an oil bath and kept for $0.5-2 \mathrm{~h}$. After cooling the mixture to room temperature and adding acetone, the aminecapped CQDS precipitated and the product was collected by centrifugation. The final solid amine-capped CQDs were obtained by dialysis and lyophilization. Another route for the synthesis of amino-coated CQDs was reported by Liu et al. ${ }^{\mathbf{1 6 4}}$ They utilized tetraethylenepentamine (TEPA) as an amine donor to synthesize amine-capped CQDs under nitrogen protection in a three-neck flask. Once TEPA was heated to $240{ }^{\circ} \mathrm{C}$, anhydrous citric acid was rapidly added to the solution under vigorous stirring. The pyrolysis reaction occurred over $3 \mathrm{~min}$ at $240{ }^{\circ} \mathrm{C}$. After dialysis and vacuum drying, the product was finally obtained.

5.1.4. Converting surface coating from carboxyl to amino groups. Amine-coated O-dots can be synthesized by converting the carboxyl groups on their surface to amino groups via a twostep process. Initially, glucose in $2 \%$ acetic acid solution was carbonized via the hydrothermal method at $180{ }^{\circ} \mathrm{C}$ for $6 \mathrm{~h}$, yielding the carboxyl-coated O-dots. To alter the surface coating after dialysis, PEG1500N was added to the carboxyl-coated O-dot solution and the mixture was heated to $120{ }^{\circ} \mathrm{C}$ for $12 \mathrm{~h}$. After dialysis, a comparatively pure solution of amine-coated O-dots was obtained. ${ }^{165}$

\subsection{Carboxyl group-coated O-dots}

Several approaches have been reported to produce carboxyl group (carboxylate)-coated C-dots, including pyrolysis, microwave irradiation, and chemical oxidation. ${ }^{\mathbf{1 6 6}}$ Accordingly, O-dots coated with carboxyl groups were prepared using CA with pyrolysis at $180^{\circ} \mathrm{C}$ for $150 \mathrm{~min}$ under normal air. ${ }^{167}$ This method was modified by Reisner et al. ${ }^{\mathbf{1 6 8}}$ who carried out the reaction for $40 \mathrm{~min}$. In this approach, further purification was not necessary because no residual citric acid was detected in the sample. A similar material was obtained using a microwave oven, in which glucose and poly(acrylatesodium) (PAAS) were dissolved in water and then heated for $4 \mathrm{~min}$. After purification, carboxylcapped C-dots were obtained. ${ }^{169}$ Chemical oxidation is another approach used for the preparation of O-dots capped with carboxyl groups. In this approach, different carbon sources, such as activated carbon or petroleum coke are employed. These materials are chemically oxidized in concentrated $\mathrm{HNO}_{3}$ or a mixture of concentrated $\mathrm{H}_{2} \mathrm{SO}_{4}$ and $\mathrm{HNO}_{3}$ at a temperature higher than $100{ }^{\circ} \mathrm{C}$ for more than 12 h. ${ }^{170,171}$ After centrifugation, the supernatant is removed and neutralized by $\mathrm{NaOH}$. Then, the carboxylate-capped O-dot solution can be collected by dialysis. Callan et al. ${ }^{172}$ changed the surface coating of O-dots from amine to carboxyl groups via a reaction occurring between the amine groups and succinic anhydride at a basic $\mathrm{pH}$. The synthesis procedure was based on two steps. Firstly, the amine-capped O-dots were prepared using carbon nanopowder refluxed in nitric acid for $12 \mathrm{~h}$. Then, the dried oxidized carbon nanoparticles were refluxed in neat $\mathrm{SOCl}_{2}$ for $6 \mathrm{~h}$. Finally, the product was reacted with bis-3-aminopropyl terminated poly(ethylene glycol), forming amino-capped O-dots. In the second step, the reaction between amine-capped C-dots and succinic anhydride led to the formation of carboxyl-capped dots. Succinic anhydride reacted with the amine capped O-dots in $\mathrm{CH}_{2} \mathrm{Cl}_{2}$ solution containing $\mathrm{Et}_{3} \mathrm{~N}$, and the reaction was continued for $15 \mathrm{~h}$ under argon at ambient temperature. Then, the mixture was neutralized with aqueous $\mathrm{HCl}$, and the carboxyl coated O-dots were extracted with $\mathrm{CHCl}_{3}$. After dehydration of the organic phase over $\mathrm{Na}_{2} \mathrm{SO}_{4}$, the solvent was removed under reduced pressure, and dark yellow carboxyl terminated O-dots were finally obtained.

\subsection{Surface coating with quaternary ammonium}

Several studies have reported the preparation of O-dots with a cationic surface coating, ${ }^{173-178}$ which are generally synthesized in three steps as follows. In the first step, a concentrated acidic solution of betaine hydrochloride was neutralized by the addition of tris(hydroxy methyl)-amino methane (Tris) at a molar ratio of $1: 1$. After this step, the water-soluble organic salts were partially removed from the solution with isopropanol. A dry viscous white precursor was produced, and subjected to pyrolysis at $250{ }^{\circ} \mathrm{C}$ in air for $2 \mathrm{~h}$. If the temperature was increased 
beyond this point, it may lead to the degradation of betaine, and thus $250{ }^{\circ} \mathrm{C}$ was adequate for the carbonization of Tris. The resultant product was extracted with water and precipitated from the colloid using acetone. After washing and drying, the surface quaternized O-dots were obtained as a dark brown powder.

\subsection{Alkoxysilane coating}

Amino-terminated alkoxy silanes including $N$-( $\beta$-aminoethyl $)-\gamma$ aminopropyl methyl dimethoxy silane (AEAPMS) ${ }^{\mathbf{1 7 9 , 1 8 0}}$ and [3-(2aminoethylamino)propyl]trimethoxy silane (AEATMS $)^{\mathbf{1 5 8 , 1 8 1}}$ have been used. These compounds were heated to $230-240{ }^{\circ} \mathrm{C}$, and then anhydrous citric acid was added quickly under vigorous stirring. In the course of pyrolysis, citric acid was rapidly decomposed and carbonized in an oxygen-free environment, which led to the formation of carbon nanoparticles with residual carboxylic acid groups. Amide bonds were obtained via reaction of the terminal $\mathrm{NH}_{2}$ on the alkoxy silanes with the residual carboxylic acid groups. The carbon nanoparticles were attached to the alkoxy silane via amide bonds. The products were purified by precipitation with petroleum ether or a mixture of toluene/hexane repeated three times.

\section{O-dots and biological materials}

As discussed previously, the reactive groups present in the surface coating of O-dots can be modified according to the requirements. Numerous organic, polymeric, inorganic or biological materials with specific functions can be attached on the surface of O-dots. Moieties can be attached to O-dots via several different interactions, including covalent bonds, electrostatic interactions and hydrogen bonds for specific applications.

\subsection{Interactions in functionalized O-dots}

Callan et al. ${ }^{172}$ reported the preparation of a CQD-NO photoreleasable nanohybrid system for the two-photon phototherapy of hypoxic tumors. This nanohybrid was produced via an amide bond formation reaction using ethyl(dimethylaminopropyl)carbodiimide (EDC) plus $N$-hydroxysuccinimide (NHS). The carboxylic acid-coated CQDs were activated by EDC/NHS in PBS at room temperature for $20 \mathrm{~min}$. Then a DMSO solution of the amine-terminated nitroaniline derivative NO photodonor was reacted with the activated carboxyl groups for $4 \mathrm{~h}$ at room temperature. The desired product was then obtained after purification. Sulfur-doped CQDs can be linked to dopamine via electrostatic interaction or hydrogen bond formation. The binding relies on the key role of $\mathrm{NH}_{2}$ groups and the aromatic ring, which occurs in plasma. This binding can be very useful for the detection of any abnormal dopamine that may be present in neurological disorders such as schizophrenia, Parkinson's disease and Huntington's disease. ${ }^{182}$ In another study, Liu et al. ${ }^{155}$ reported a multifunctional platform for serum-resistant gene delivery and bioimaging based on O-dots modified with a poly cationic$b$-poly-zwitterion copolymer using an atom transfer radical

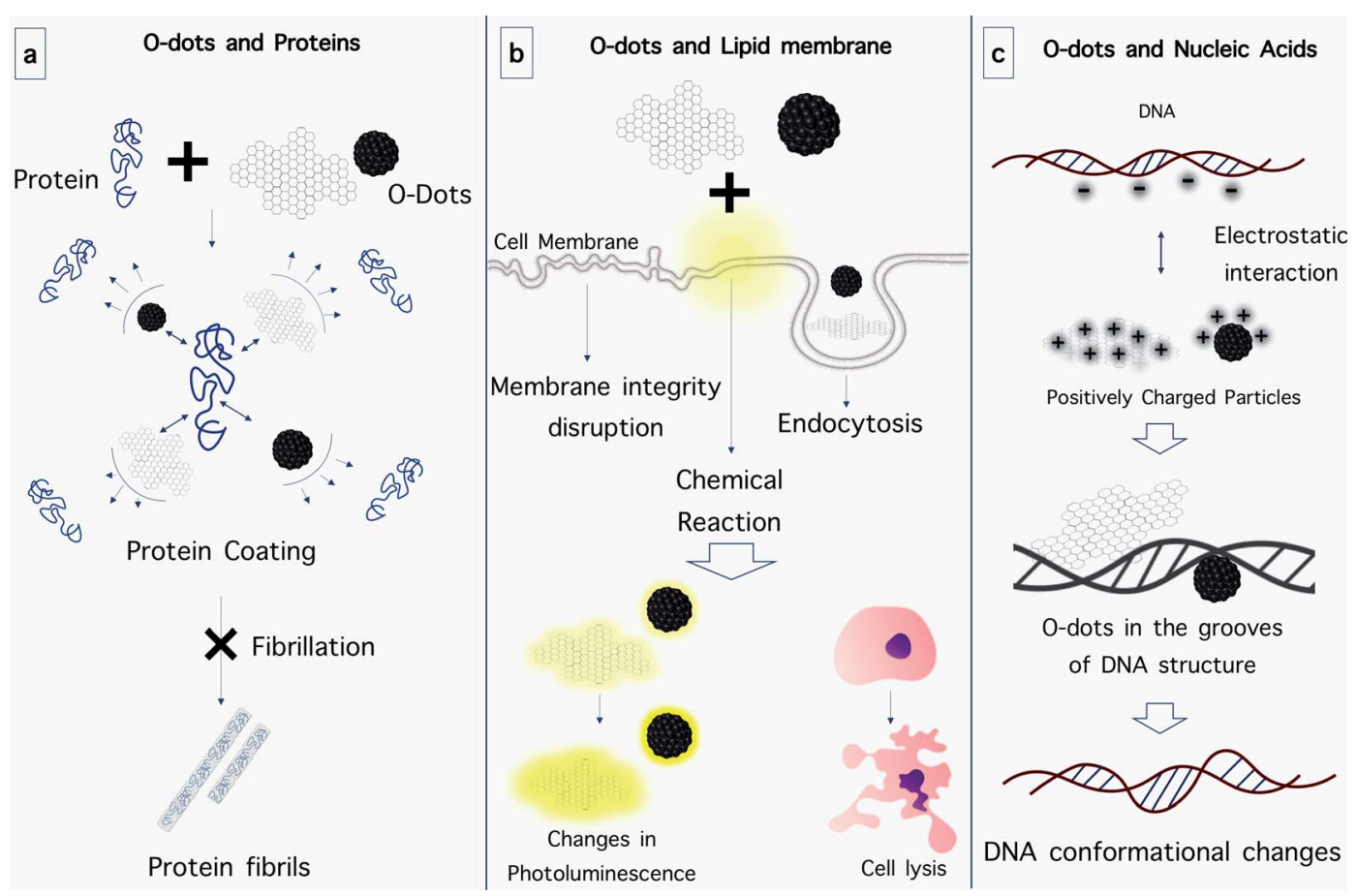

Fig. 14 Interaction of $\mathrm{O}$-dots with biological components. (a) $\mathrm{O}$-dots prevent protein fibrillation by preventing the interaction of protein subunits with each other. (b) O-dots cause disruption in cellular membrane integrity, enter cells via endocytosis and cause chemical variations in membranes, leading to cell lysis and changes in photoluminescence. (c) O-dots attach to nucleic acids via electrostatic interaction and make conformational changes in them. 
polymerization (ATRP) reaction. In this method, the PDMA/Odots were prepared according to the following procedure. Bromine-functionalized CQDs, 2-(dimethylamino)-ethyl methacrylate (DMAEMA), $\quad N, N, N^{\prime}, N^{\prime \prime}, N^{\prime \prime}$-pentamethyldiethylenetriamine (PMDETA), and $\mathrm{NaCl}$ were added to a degassed ethanol suspension of $\mathrm{CuCl}_{2}$ under $\mathrm{N}_{2}$ protection, and the reaction was continued for $3 \mathrm{~h}$ at room temperature. An aqueous solution of MPDSAH was then injected into the mixture and the reaction was allowed to proceed at room temperature for $24 \mathrm{~h}$. The solution was dialyzed against deionized water for 7 days to completely remove the impurities, and was subsequently freeze dried. Anhydrous polymermodified CQDs (PDMA-PMPD/CQDs) were eventually obtained.

Functional groups can also be attached to the surface of $\mathrm{O}$ dots via hydrogen bonds. For instance, Ouyang et al. developed a novel turn-on fluorescence probe for the targeted imaging of cancer cells via the hydrogen bond interactions between folic acid (FA) and carboxyl-coated CQDs. The probe was synthesized by mixing FA and CQDs in an aqueous solution, which was dialyzed three times against $\mathrm{pH}$ 7.4 PBS buffer for 2 h. $^{169}$ In another study, bi-functional protein nano fiber (PNF)-GQD nano-hybrids based on non-covalent interactions $(\pi-\pi$ and electrostatic) between motif-designed PNFs and fluorescent GQDs were synthesized. Because these PNF-GQD nano-hybrids possessed both a recognition moiety (RGD peptide) and a fluorescent imaging probe (GQD), they could simultaneously target and image tumor cells. ${ }^{183}$

\subsection{Interactions of $\mathrm{O}$-dots with biomolecules}

6.2.1. O-dot interaction with protein fibrils. The formation of peptide or protein fibrils within the extracellular space of tissue (Fig. 14a) is believed to play a significant role in the development of serious diseases such as Alzheimer's disease, Parkinson's disease and type-II diabetes. ${ }^{\mathbf{1 8 4 , 1 8 5}}$ The formed mature protein fibrils are known to be cytotoxic and can provoke the death of affected cells. ${ }^{186}$ Therefore one preventative or therapeutic strategy for diseases associated with peptide or protein fibrillation is to inhibit or delay the fibrillation process. Various components including organic molecules, ${ }^{\mathbf{1 8 7}}$ functional polymers, ${ }^{188,189}$ QDs, ${ }^{190,191}$ and carbon-based nanomaterials ${ }^{192}$ have been explored to inhibit peptide or protein fibrillation. The possible application of O-dots for the inhibition of protein fibrillation was recently reported. Specifically, one new type of CQDs was prepared by Li et al. ${ }^{193}$ who investigated their effect on the fibrillation of human insulin. In this study, the formation of insulin fibrils was significantly delayed when CQDs were added in a dose-dependent manner. No insulin fibrils were observed after incubation at $65^{\circ} \mathrm{C}$ for 5 days (i.e. 120 h) when a large concentration of CQDs $\left(40 \mu \mathrm{g} \mathrm{mL} \mathrm{m}^{-1}\right)$ was present. Moreover, when the CQDs were covalently attached to other proteins (such as transferrin, human serum albumin, chicken ovalbumin, and hemoglobin), no protein fibrillation or conformational alteration was detected, even after $48 \mathrm{~h}$ at ambient temperature. ${ }^{194}$ Mechanistically, the inhibitory effect of CQDs on insulin fibrillation was most efficient when added in the early stages, showing that the inhibitory effect was possibly due to the interactions between the CQDs and insulin species (monomers or oligomers) before a critical nucleation concentration was reached. ${ }^{195}$ The inhibitory effects of CQDs on human islet amyloid polypeptide (hIAPP) and amyloid peptide $\beta$ 33-42 (a key fragment of $A \beta 42$ ) have also been reported. ${ }^{196}$ Interestingly, O-dots prepared by different synthetic routes can exhibit different inhibitory effects. In a recent study, hIAPP fibrillation was inhibited by CQDs produced from organic material precursors, while the CQDs prepared from graphene oxide (GO) were surprisingly shown to stimulate the formation of fibrils. ${ }^{196}$ The different effects on amyloid fibrillation were ascribed to the differences in the structure and surface properties of these CQDs. Architecturally, the CQDs obtained from organic precursors tend to be spherical particles with plentiful nitrogen/ oxygen-based functional groups on their surface. The strong interaction among these hydrogen-bond donating groups and the backbone groups of the peptide was proposed to prevent hIAPP from ripening into fibrils due to the electrostatic repulsion of the charged particles against each other. By contrast, for CQDs synthesized from GO sheets, hydrogen bonds between the backbone of the peptide and the CQDs were less likely to occur due to the steric effects from the large hydrophobic edges on GO. Furthermore, these CQDs also inherited the layered structure from GO, which could strongly absorb peptides via their aromatic and hydrophobic residues. The strong $\pi-\pi$ and hydrophobic interactions between the peptide chains and the CQDs increased the local peptide concentration, and destabilized the folded state, which promoted the aggregation of the peptides. ${ }^{197}$

Fluorinated graphene quantum dots (F-GQDs) are a new type of carbon nanomaterials with unique physicochemical properties due to their highly electronegative fluorine atoms. Yousaf et al. prepared highly fluorescent and water-dispersible F-GQDs using a microwave-assisted hydrothermal method, and investigated their inhibitory effect on the aggregation and cytotoxicity of hIAPP in vitro. The efficient inhibition of amyloid aggregation by the addition of F-GQDs was confirmed. In the presence of F-GQDs, the morphology of the hIAPP aggregates changed from entangled long fibrils into short thin fibrils and amorphous aggregates. By employing fluorescence analysis using thioflavin $\mathrm{T}$, inhibited aggregation with a prolonged lag time, and reduced fluorescence intensity at equilibrium were demonstrated after hIAPP was incubated with the F-GQDs. Based on the circular dichroism spectrum results, the F-GQDs could inhibit the conformational transition of the peptide from its native structure to $\beta$-sheets. F-GQDs could also rescue the cytotoxicity of INS-1 cells induced by hIAPP in a dosedependent manner. ${ }^{198}$

6.2.2. O-dot interaction with lipids. Another topic of interest is the possible interaction between O-dots and lipids or bio-membranes (Fig. 14b). For many applications such as bioimaging, diagnosis, and drug delivery, the interactions between O-dots and lipids/or bio-membranes are of great significance because O-dots may hypothetically influence the structure and properties (e.g. membrane permeability) of biological membranes. Recently, it was shown that CQDs could bind to the lipid bilayer and enter through cell-sized giant 
unilamellar vesicles. ${ }^{199}$ Jelinek and colleagues made significant contributions to elucidate the interaction of CQDs with lipids/ membranes. ${ }^{\mathbf{2 0 0 - 2 0 2}}$ In one study, they showed that amphiphilic CQDs could insert into the lipid bilayer of giant vesicles and induce FRET energy transfer from the CQDs to dyes embedded in the membrane. ${ }^{203}$ By conjugating the amphiphilic CQDs with phospholipids, the same group developed a new probe for the study of the environment and processes occurring in membranes. ${ }^{204}$ The interactions between the amphiphilic CQDs with actual bacterial cell membranes (not models) were also studied, in which the emission spectra of CQDs depended on the identity of the bacterial strains with regard to the spectral shift and peak intensity. This may offer an opportunity to identify different species of bacteria using fluorescence spectroscopy and microscopy. ${ }^{205}$ Furthermore, the unique interaction of CQDs with bacterial membranes was exploited to detect bacteria in a biological environment by a different group. ${ }^{206}$ Recently, Pan and co-workers reported a very interesting study, whereby the interaction of CQDs with the cell membrane was exploited to design "caged" CQDs for monitoring intracellular trafficking via switchable PL. ${ }^{204}$ In this study, negatively-charged highly luminescent "bare" CQDs showed reduced PL intensity if positively charged macromolecules became wrapped around their surface. Nevertheless, the "quenched" CQDs could recover their emission through interaction with anionic surfactant molecules (i.e., anionic amphiphiles or endocytic membranes) and this phenomenon could be used for monitoring intracellular trafficking.

Studies on the antibacterial activity of CQDs have also been reported recently. ${ }^{207}$ The antibacterial activity of these C-dots was attributed to the destructive interactions between CQDs with the bacterial cell membranes, which closely depended on the surface charge on the CQDs. ${ }^{208}$ To elucidate the cellular behavior of CQDs, Zhou and coworkers ${ }^{207}$ carried out a systematic study. In this study, it was found that the cellular uptake of CQDs was a dose-, time- and partially energy-dependent process, which also to some extent involved passive diffusion. These CQDs could penetrate the cell membranes by endocytosis via caveolae-mediated and clathrin-mediated pathways.

Similar to CQDs, graphene quantum dots can also alter the structure of cell membranes. Jiang and co-workers assessed the toxicity of graphene oxide (GO) and nitrogen-doped graphene quantum dots (N-GQDs) on red blood cells (RBCs) through analysis of hemolytic activity. The morphology of the RBCs changed and their ATP content was lower after being exposed to the different nanomaterials. The structural changes of the RBC lipid membranes were studied via surface-enhanced infrared absorption spectroscopy using model membranes. The analysis of the infrared spectra confirmed that the adsorption of GO perturbed the integrity of the membrane by extracting the lipid bilayer, resulting in hemolysis and aberrant-shaped cells. By contrast, when N-GQDs were tested, the disturbance of the structure and conformation of the lipid was less, which resulted in fewer abnormal cells. ${ }^{209}$

6.2.3. O-dots interaction with nucleic acids. It is important to examine the interaction between nucleic acids (DNA and RNA) and different nanomaterials because nucleic acids are responsible for carrying genetic information (Fig. 14c). Recently, the interaction of CQDs with different types of DNA structures, i.e. double-stranded DNA (dsDNA) and singlestranded DNA (ssDNA) has attracted interest. Studies have shown that two different interaction mechanisms with CQDs can occur, producing discrete changes in fluorescence behavior. ${ }^{210-212}$ As an example, Loo et al. prepared a sensing platform in which CQDs and a fluorescently labeled ssDNA probe (FAMLprobe) were used to identify a specific sequence of DNA. ${ }^{209}$ Due to the difference in the interactions of CQDs with SSDNA and dsDNA, the fluorescence of the FAMLprobe (ssDNA) was effectively quenched by CQDs, unless it had formed dsDNA by base-pairing with the target DNA.

It is expected that the negative charge of nucleic acids caused by the phosphate backbone will interact strongly with positively-charged CQDs through electrostatic interactions. ${ }^{213}$ The interaction of CQDs with DNA may be so strong that it can alter the configuration of DNA. Sun and co-workers found that positively-charged CQDs selectively promoted the transformation of right-handed DNA (B-DNA) into left-handed DNA (Z-DNA). ${ }^{210}$ It is believed that in this interaction, CQDs bind inside the main groove of DNA, as verified by competitive binding experiments using ethidium bromide, daunomycin, and Hoechst 33258. It is worth noting that Z-DNA has been identified as a transient structure that is activated by normal biological processes such as transcription, DNA supercoiling and cytosine methylation. ${ }^{215,216}$ Based on this finding, some DNA-based logic gates have been designed to exploit the fluorescence resonance energy transfer between CQDs and DNA intercalators. With their high biocompatibility, superior optical properties and predictable interactions with nucleic acids, CQDs have also found important applications as delivery vehicles for nucleic acids in a highly efficient manner. ${ }^{214}$ A study was reported using fluorescent CQDs as an efficient nano-carrier for siRNA to enable gene knockdown in gastric cancer cells with promising results. ${ }^{215}$

As mentioned above, the phosphate groups of DNA provide a negative charge, and thus the positive charge on the surface of O-dots is very important for suitable electrostatic interaction. Without this modification, the interaction between O-dots and DNA is probably too weak, and thus will lead to rapid release of therapeutic genes during delivery. Thus, cationic polymers such as polyethylenimine (PEI) are widely used to provide a positive charge. ${ }^{216}$ In 2017, the Yang group prepared a gene delivery system using CQDs, equipped with PEI and folic acid (FA). They used gel electrophoresis to ensure the ability of CQDs with PEI to bind to DNA due to their positive charge. It was concluded that the composite could be used to transfer plasmid DNA into cells. $^{217}$

A recent study on the interaction of GQDs with three different types of DNA (well-matched DNA (WM-DNA), baseimpaired DNA (AB-DNA) and amino-modified DNA (AM-DNA)) showed that GQD could reduce the melting temperature of DNA, and thus reduce its stability. It was also observed that the WM-DNA structure and conformation were not changed, but AB-DNA changed after incubation with GQD. ${ }^{218}$ 


\subsection{The interaction of O-dots with the blood-brain barrier} (BBB)

In many applications, especially for drug and gene delivery systems, it is important to investigate the behavior of O-dots in biological environments. One of the important organs in the human body is the brain. In recent studies, scientists have concentrated on the ability of O-dots to cross the blood-brain barrier (BBB), the most important barrier in the central nervous system (CNS) (Fig. 15b). The BBB acts as a physical barrier and regulates the passage of designated molecules between the bloodstream and the brain by either paracellular or transcellular pathways. The BBB hinders the diffusion of many therapeutic agents from the blood into the brain. The tight junctions in the BBB have pores with a size of 4-6 nm. Therefore, nanoparticles that are $4 \mathrm{~nm}$ or less in size can cross the BBB by passing through these gaps. ${ }^{219,220}$

The mechanisms of BBB penetration can be divided into active and passive transport routes. ${ }^{221}$ The passive transport route is an energy-independent process, for instance, simple diffusion. Frequently, the passive diffusion of drugs used against tumors occurs via the enhanced permeability and retention effect (EPR). ${ }^{222}$ The active transport routes include receptor-mediated and adsorption-mediated endocytosis and carrier-mediated transport, all of which require energy obtained by the hydrolysis of adenosine triphosphate (ATP). ${ }^{223}$ Besides, the transportation of drugs can be enhanced by different NPs and their specific mechanisms. For instance, the small stereospecific pores that are employed in the carrier-mediated transport system restrict the movement of large-molecule drugs. ${ }^{224}$ However most CDs have an ultra-small size (1-10 nm) and versatile surface functionalities, ${ }^{225}$ which can improve large molecule delivery if the CDs are covalently conjugated to the drug. ${ }^{226}$

Pediatric malignant glioma is one of the most common cancers among children, which is be fatal. Doxorubicin (Dox) is

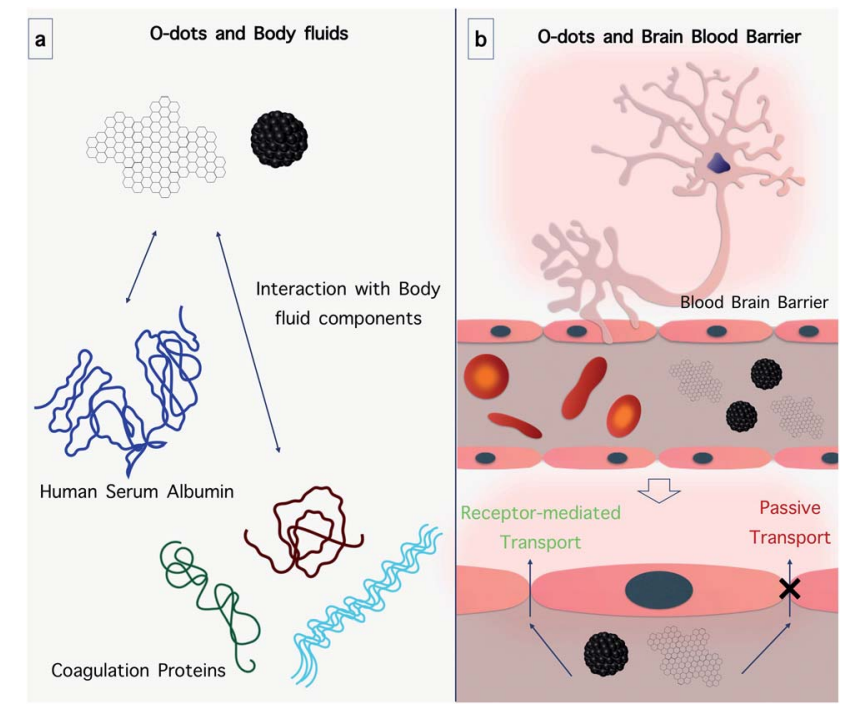

Fig. 15 Interaction of $\mathrm{O}$-dots with body fluids and the brain blood barrier. (a) $\mathrm{O}$-dots can interact with body fluid components, specifically proteins. (b) O-dots can pass through the BBB via passive and active transport. an effective drug that can hinder the replication of many different cancer cells. Nonetheless, Dox frequently causes severe side effects due to its non-specific attack on healthy cells, such as congestive heart failure. Thus, the delivery and release of drugs such as Dox across the BBB into the CNS can selectively target pediatric brain tumors. Li et al. covalently linked CQDS with transferrin to efficiently deliver Dox due to the overexpression of the transferrin receptor (TfR) on the BBB and cancer cells. CQDs were prepared from raw carbon powder using a "top-down" approach. ${ }^{227}$ Then SJGBM2 cells, a pediatric brain tumor cell line, were used to demonstrate the higher uptake of the conjugate compared Dox alone, which was confirmed by counterstaining with Hoechst. Four different tumor cell lines (CHLA-266, CHLA-200, SJGBM2 and Daoy) were exposed to 1, 10 and $100 \mathrm{nM}$ of the conjugate and Dox aqueous solutions. The results showed that $10 \mathrm{nM}$ conjugate solution gave the highest toxicity to all the pediatric tumor cell lines.

In the same year, based on publications that showed the importance of transferrin in transporting drugs to the brain parenchyma $^{228}$ and brain glial cells, ${ }^{229,230} \mathrm{Li}$ and coworkers employed zebrafish as a biological model to investigate whether transferring-conjugated CQDs could cross the BBB. ${ }^{231}$ Zebrafish is a vertebrate species with a high physiological similarity to humans and genetic homology. ${ }^{232}$ The advantages of zebrafish include their easy culture, transparent body, and the rapid formation of the BBB. Zebrafish are considered to be a superior model to mice for studying whether CQDs can pass across the BBB using transferrin-receptor mediated endocytosis. ${ }^{\mathbf{2 3 3 , 2 3 4}}$

CQDs and the CQD/Trans/Dox conjugate were directly injected into the zebrafish heart. However, there was no fluorescence in the CNS when CQDs were injected alone. To show that the lack of fluorescence did not result from the low quantum yield of CQDs, a dye (5-(aminomethyl)fluorescein) was conjugated with the CQDs. The conjugated CQDs with 5-(aminomethyl)fluorescein also did not show any fluorescence in the CNS of the zebrafish. Therefore, it was concluded that CQDS alone could not cross the BBB. Similarly, the CQD/Trans/Dox conjugate was conjugated to a dye to enhance its PL emission. The CQDs/Trans/Dox conjugate labeled with the dye was successfully shown to reach the CNS by fluorescence spectroscopy, suggesting that the CQDs could cross the BBB, probably by TfR-mediated endocytosis.

Although CQDs are promising drug carriers, to some extent they possess the same limitations in crossing the blood-brain barrier as small molecule drugs. If CQDs can be prepared from a precursor that is known to be able to cross the $\mathrm{BBB}$, there is a chance that the precursor molecules on the CQD surface can also allow CQDs to reach the brain. In the study by Mintz et al., tryptophan CQDs were produced from tryptophan, which is an amino acid that can cross the BBB via LAT1 transportermediated endocytosis. ${ }^{235}$ Two types of CQDs were synthesized using tryptophan and two additional nitrogen dopants, namely urea and 1,2-ethylenediamine. As mentioned previously, these CQDs were able to cross the BBB of zebrafish (Danio rerio) via the LAT1 transporter.

In another study, GQDs were used to hinder the fibrillization of $\alpha$-synuclein ( $\alpha$-syn), and could interact directly with mature 
fibrils, causing their disaggregation. Moreover, GQDs could prevent neuronal cell death and synaptic loss, decrease the formation of Lewy bodies and Lewy neurites, reverse mitochondrial dysfunction, and prevent the neuron-to-neuron transmission of preformed $\alpha$-syn fibrils. ${ }^{5,6}$ In vivo studies showed that GQDs could penetrate the BBB and protect against dopaminergic neuron loss induced by preformed $\alpha$-syn fibrils, and reduce Lewy body/Lewy neurite pathology and behavioral deficits. ${ }^{236}$

\subsection{O-dot interactions with biological fluids}

The behavior of O-dots in various biological and body fluids is very important since most reactions in humans and other living organisms occur in a fluid environment (Fig. 15a). Injection of nanoparticles intravenously or subcutaneously is the most common route of administration, and therefore exhaustive testing is required to determine the fate of nanoparticles within the blood circulation (the main fluid within the body) and their impact on hematopoietic functions and cells. ${ }^{237}$ Moreover, studies in simulated physiological condition such as blood at $\mathrm{pH} \sim 7.4$ and temperature $\sim 37{ }^{\circ} \mathrm{C}$ have shown that CQDs can be efficient drug delivery systems. As an example, researchers increased the release rate of Dox in physiological fluid by linking carboxymethyl cellulose-hydroxyapatite to CQDs, compared to the composite without CQDs. ${ }^{238}$ Therefore, it seems that CQDs are effective drug delivery systems in physiological environments. The study of the interaction of CQDs with plasma components, especially coagulation-related proteins, is crucial in the field of drug delivery. In 2017, the effect of O-dots derived from Schizonepetae Herba Carbonisata (SHC) on a liver wound was investigated. The animals treated with O-dots had a shorter bleeding time compared with the control group. However, the study of O-dot behavior in plasma requires more research. ${ }^{239}$ The most abundant protein in plasma is serum albumin. In 2018 Guo and coworkers examined the behavior of O-dots in the presence of human serum albumin (HSA). The results showed that the O-dots interacted with HSA through hydrophobic interactions and hydrogen bonds. A bond was created between the oxygen or nitrogen atoms in the amino acids present in HSA and nitrogen and oxygen-containing functional groups in the $\mathrm{O}$ dots. This binding could change the HSA secondary structure and disturb the normal function of the protein. ${ }^{240}$

\section{O-dots as a theranostic platform}

The distinctive optical features of O-dots make them highly attractive candidates as imaging reporters combined with drug delivery and therapeutic applications. Their special properties allow them to overcome various problems associated with conventional imaging probes and to provide versatile nanoplatforms with both imaging and therapeutic capabilities. Herein, we survey the applications of O-dots for theranosticenabled drug delivery and therapy (Fig. 16).

\subsection{Non-targeted cargo delivery}

Although currently there is a trend toward targeted cargo delivery, non-targeted systems are still useful in some biomedical applications. Moreover, a need for systemic effects of drugs is another reason. Also, making cargo carriers responsive to certain stimuli can also be a suitable alternative to targeted cargo carriers.

CQDs-Dox antitumor drug complexes were synthesized using a combination of CQDs and Dox through a hydrothermal method. The surface functional groups of CQDs were connected to Dox through electrostatic interactions, and the CQDs-Dox complexes could be transported into cells via both endocytosis and passive diffusion. Moreover, these complexes exhibited $\mathrm{pH}$ sensitive Dox release behavior, and enhanced antitumor activity compared to Dox alone. ${ }^{241}$ In another study, hollow CQDs with a diameter of $c a .6 .8 \mathrm{~nm}$ and pore size of $c a .2 \mathrm{~nm}$ were investigated as a delivery carrier for Dox. The Dox-hollow CQD drug delivery system (DDS) could be rapidly internalized by cells, and the release of the drug was regulated by $\mathrm{pH}$. These hollow CQDS showed promise for both cell imaging and cancer therapy due to their nanostructure and photoluminescence properties. ${ }^{242} \mathrm{Li}$ and co-workers prepared CQDs from ginger as a precursor, which were efficient without any drug cargo to kill hepatocellular carcinoma cells (HepG2) and showed lower toxicity towards normal mammary epithelial cells (MCF-10A) and normal liver cells (FL83B). ${ }^{243}$ They prepared the ginger CQDs via the simple hydrothermal treatment of ginger juice. Their CQDs selectively killed HepG2 cancer cells over other cancer cells, including human lung cancer cell line (A549), human breast cancer cell line (MDA-MB-231) and human cervical cancer cell line (HeLa). Western blot analysis revealed that the CQDs upregulated the expression of p53 protein only in the case of HepG2 cells. In addition, they found that the uptake of CQDs by HepG2 cells increased the intercellular production of reactive oxygen species (ROS) up to 18.2-fold, which led to the induction of apoptosis, whereas in other cells, the ROS level was almost unchanged after treatment with $1.11 \mathrm{mg} \mathrm{mL}^{-1}$ CQDs. A subsequent in vivo study in a nude mice tumor model showed that the CQDs could effectively inhibit the growth of tumors $(3.7 \pm 0.2$ vs. $104 \pm 14 \mathrm{mg}$ with and without treatment after 14 days).

Moreover, Yuan et al. reported that CQDs prepared from milk showed pH-dependent release behavior of Dox. Briefly, the negatively charged Dox was effectively bound to the positively charged CQDs. Compared with the free Dox, CQD-Dox demonstrated greater cytotoxicity towards cancer cells, whereas it showed lower toxicity towards a normal mouse fibroblast cell line. ${ }^{244}$

Khodadadei et al. produced $10 \mathrm{~nm}$-sized nitrogen-doped GQDs (N-GQDs) with 10 graphitic layers, which could be loaded with methotrexate (MTX) to create a drug delivery system. The results showed that the GQDs were robust nanocarriers with higher anti-tumor activity, and could extend the cytotoxic effects of the loaded drug for a longer time. ${ }^{245}$ Some et $a .^{246}$ used curcumin (Cur) as an anti-cancer drug. They showed that curcumin could attach to GQDs by interacting with the polar oxygen-containing groups at the edge of the GQDs. They found that the presence of more surface oxygen functional groups increased the loading capacity of the GQDs for curcumin. This occurred at $\mathrm{pH}$ 9, which is much higher than the physiological $\mathrm{pH}$ of 7.4 , indicating that Cur was unlikely to 


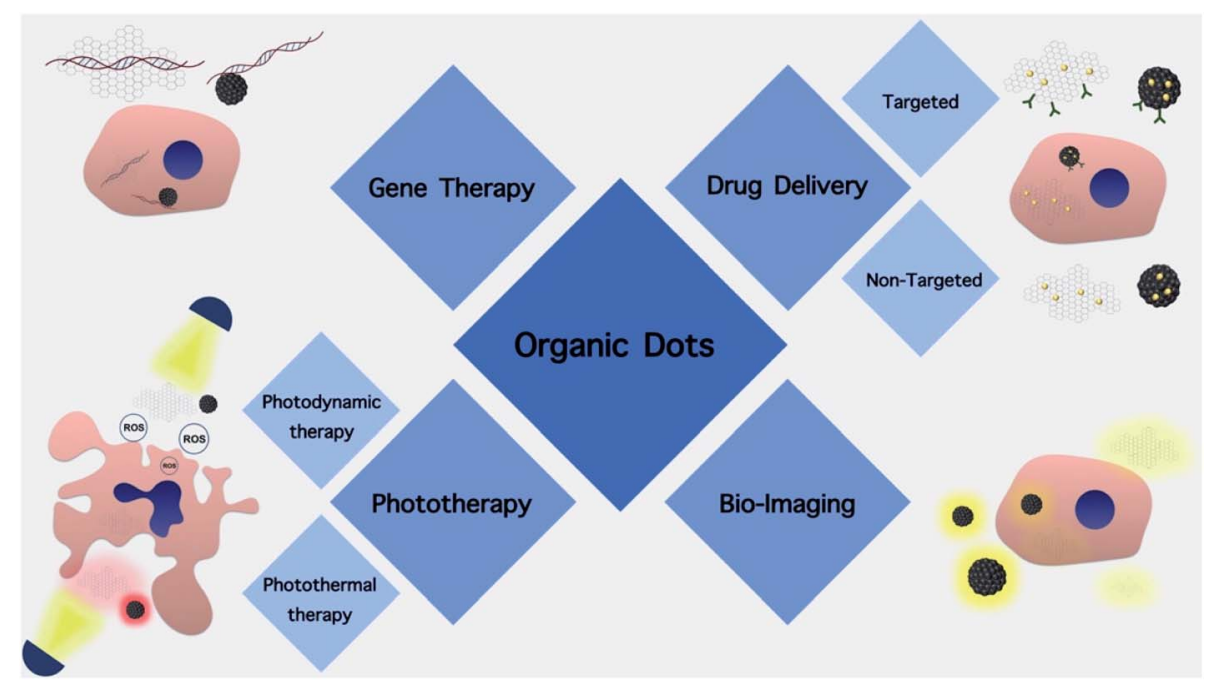

Fig. 16 Schematic of the biomedical applications of organic dots.

dissociate in the extracellular environment or in normal healthy cells. They observed dissociation of Cur from the GQDs at the lower $\mathrm{pH}$ values typical of cancer cells. Up to $85 \%$ was released within $24 \mathrm{~h}$ at $\mathrm{pH} 5$, compared to only $5 \%$ at pH 9 and $9.8 \%$ at $\mathrm{pH}$ 7.5. The GQD-Cur complex exhibited good ability to kill human colon adenocarcinoma HCT 116 cells, killing $90 \%$ at 100 $\mu \mathrm{g} \mathrm{mL} \mathrm{m}^{-1}$, compared with $70 \%$ for Cur alone. The in vivo tests showed a much lower tumor volume and mass after 14 days in the mice with HCT tumors. The tumor volume for the mice treated with GQD-Cur was $200 \mathrm{~mm}^{3}$ (almost no increase in tumor size) compared to $1000 \mathrm{~mm}^{3}$ for the control mice.

\subsection{Targeted cargo delivery}

Some of the side effects of treatments are due to their unspecific functions and targets. Many drugs can be taken up by other cells rather than the intended cells. Moreover, this unspecific uptake also causes low concentrations of drugs in the intended sites, which would be below the therapeutic levels, and therefore, the real targets would not uptake a sufficient amount of drug. In this case, the primary concentration of the drug has to increase in order to provide a sufficient concentration at the diseased sites. This problem indicates a need for more specific therapy methods. Since biological systems demonstrate specific characteristics and properties, it is possible to target them for better therapies and treatments. Moreover, a suitable platform is needed for functionalization to become sensitive, specific and responsive to biological systems besides loading cargo. O-dots have shown promising properties in the field of targeted cargo delivery due to their capacities in being hybridized and functionalized properly with other nanoparticles, polymers, biomolecules, etc.

Individual O-dots contain various functional groups on their surface. For example, the carboxyl groups of O-dots can be functionalized by $\mathrm{N}$-hydroxysuccinimide (NHS) esters to conjugate with lysine residues in protein structures. O-dots have been used to label actin for actin polymerization studies, transferrin receptors were probed with fluorescent O-dot-labeled transferrin, and albumin-O-dot tags have been used for in vitro and in vivo signaling studies. This strategy is most suitable for CQDs with a high QY, which are prepared from citric acid with carboxyl functional groups. The conjugation of proteins with CQDs generally leads to an increase in fluorescence intensity and lifetime owing to the reduction of intramolecular dynamic fluctuations. Moreover, protein tagging can lead to less fluctuations in the fluorescence and more efficient use of excitation photons, which can be determined by single-molecule fluorescence measurements. ${ }^{247,248}$

The most common non-covalent interactions of CQDs occur between organic molecules or fluorophores. These interactions are hydrogen bonds, dipole-dipole interactions, hydrophobic interactions, van der Waals forces, electrostatic forces, and $\pi-\pi$ stacking. Moreover, several factors such as $\mathrm{pH}$, metal ions and organic solvent play a critical role in the attachment of organic molecules and fluorophores to CDs. ${ }^{249}$ P-CQDs/HA-Dox is one example of non-covalent interactions between positively charged polyethylene imine-modified CQDs (PEI-CQDs) and negatively charged hyaluronic acid (HA)-conjugated doxorubicin (Dox). These nano-hybrids were prepared via an electrostatic self-assembly method and used for the detection of hyaluronidase (HAase) activity in drug delivery. ${ }^{250}$ Moreover, PEI-CDs could also form a complex with folic acid (FA) via noncovalent binding, which was an effective approach for detecting and targeting folate-receptor (FR)-positive cancer cells. The fluorescent CQDs were produced using a hydrothermal method, and their surface was coated by positively-charged polyethylene imine (PEI), which allowed further conjugation with FA by electrostatic interaction. The FA-targeted PEI-modified CQDs (FA-PEI-CQDs) could act as a turn-on fluorescent nanoprobe in FR-positive cancer cells. ${ }^{251,252}$ Furthermore, the conjugation of PEI with CQDs led to positive charges on the CQD surface, which facilitated electrostatic interactions between the CQDs and DNA. ${ }^{253}$ Carboxyl-rich CQDs can be alkynylated using an amidation reaction with propargylamine in the presence of $1,1^{\prime}$ - 
carbonyldiimidazole. Furthermore, $\mathrm{Cu}_{2-x} \mathrm{~S}_{y} \mathrm{Se}_{1-y} \mathrm{NCs}$ have been used as catalysts in the click reaction between alkynylated CQDS and azido-DNA for the preparation of modified CQDs. Covalently bonded DNA-CQDs can be produced by a copper(I)-catalyzed alkyne-azide cycloaddition click reaction (CuAAC) catalyzed by $\mathrm{Cu}_{2-x} \mathrm{~S}_{y} \mathrm{Se}_{1-y} \mathrm{NCs}$. Research showed that DNAmodified CQDs are useful in biosensing and bioimaging approaches. ${ }^{\mathbf{8 8 2 5 4}}$ Aptamer-based spectrofluorometric methods provide a sensitive approach for the detection of cancer cells. CQDs modified with the AS1411 aptamer act as a sensitive and selective signal enhancer for the spectrofluorometric detection of various target cancer cells. AS1411is a nucleolin-specific aptamer for the detection of nucleolin, which is overexpressed on the surface of cancer cells. The aptamer molecules are physically wrapped around CQDs. Incubation of target cancer cells in a CQD-aptamer solution led to an enhancement in the fluorescence intensity. ${ }^{255,256}$ The functionalization of CQDs with the nuclear localization signal peptide (NLS-CQDs) is a useful strategy for the transportation of Dox into cancer cells to improve the anticancer effect of this drug. Dox was covalently conjugated with NLS-CQDs (Dox-CQDs) via an acid-labile hydrazine bond, which was degraded in the weakly acidic intracellular milieu and showed superior suppression of tumor growth. ${ }^{257,258}$ A system of $\mathrm{pH} /$ redox dual-responsive CQDs (CQDs-RGD-Pt(Iv)-PEG) could act as fluorescent imaging targeted nanocarriers. RGD peptide acted as an active targeting ligand and cisplatin(Iv) as a prodrug. They were used for extracellular-triggered tumor targeting for improved anticancer drug delivery. The hydrolysis of the benzoic-imine bonds at the low extracellular $\mathrm{pH}$ led to exposure of the inner targeting RGD peptide, followed by effective uptake by interaction with the integrin $\alpha v \beta 3$ (ligand-receptor) on the cancer cells. ${ }^{259-261}$ Polymer-coated CQDs (p-CQDs) are a new class of fluorescence nanoprobes with an ultra-small size, good biocompatibility, and high water solubility, which can be synthesized via direct solvothermal transformation. They can be covalently conjugated with I6P8, which is a fragment of interleukin-6 (IL-6), identified by the phage display technique, and can specifically bind to the IL-6 receptor. This receptor is expressed on both brain capillary endothelial cells and various cancer cells such as glioma. Conjugation of p-CQDs with I6P8 (cys-I6P7) led to improvements beyond that expected from passive targeting. The encapsulation of drugs such as Dox can be stabilized by these nanocarriers, allowing them to cross the BBB and deeper penetration of drugs into brain tumors. In summary, blocking the oncogenic function of IL- 6 and the pH-responsive release of the loaded Dox are the main mechanisms for the improved anticancer effects. ${ }^{262,263}$ A nuclear-targeted drug delivery vehicle was fabricated by immobilizing a nuclear localization signal peptide (NLS) onto CQDs (NLS-CQDs). Through an acid-labile hydrazone bond, Dox was coupled onto NLS-CQDs (NLS-CQDs-Dox) and released in the weakly acidic intracellular compartment. NLS-CQD-Dox was mainly located in the nucleus and could efficiently induce apoptosis in human lung adenocarcinoma A549 cells. Moreover, NLS-CQD-Dox exhibited enhanced ability to inhibit tumor growth in vivo compared with free Dox in an A549 xenograft nude mouse model. ${ }^{264}$
Folic acid (FA)-conjugated GQDs could also be loaded with Dox. This nanoassembly could unequivocally distinguish cancer cells from normal cells, and efficiently deliver the drug to target cells. The inherently stable fluorescence of GQDs enabled real-time monitoring of the cellular uptake of the Dox-GQD-FA nanoassembly and the consequent release of Dox. The nanoassembly was rapidly internalized by HeLa cells via receptormediated endocytosis, while the Dox release and accumulation were improved. ${ }^{265}$

Justin et al. ${ }^{266}$ used GQDs as a non-toxic imaging agent to track the diffusion of drugs from microneedle arrays. Lidocaine hydrochloride (LH) was attached by $\pi-\pi$ stacking to the GQDs and could passively diffuse through the microneedles due to its small molecular weight. They also coated GQDs with bovine serum albumin (BSA) as a large molecular weight cargo. GQDLH also become loaded into chitosan to be used in a microneedle array for delivery. They used iontophoresis to stimulate the release of the BSA, which showed an increase in release rate from to $94.5 \%$ compared to $7.6 \%$ by passive diffusion.

Rare earth up-conversion nanoparticles and GQDs have attracted much interest in biomedicine, but they all have some inherent limitations as nanocarriers for in vivo drug delivery. One study reported a new concept for the fabrication of a GQDrare earth up-conversion hybrid via coordination between rare earth metal ions and histidine, and attachment to octadecylamine-functionalized GQDs with fluoride ion replacement and subsequent hydrothermal treatment. The hybrid had a cage-like nanostructure with a mean particle diameter of $46.2 \mathrm{~nm}$, comprising several layers of graphene sheets plus $\mathrm{NaYF}_{4}: \mathrm{Yb}, \mathrm{Tm}$ nanocrystals. It showed high stability in physiological buffer and 140-fold enhancement of upconversion luminescence. This biomimetic drug delivery system was composed of the hybrid plus gold nanoparticles as the core and MGC-803 cell membranes as the shell, with a Dox loading capacity of $461.2 \%$. It showed both $\mathrm{pH}$ and lightresponsive drug release and carried out chemo/photothermal therapy with higher anticancer activity than free Dox. ${ }^{\mathbf{2 6 7}}$

In another study, a composite of GQDs and $\mathrm{Fe}_{3} \mathrm{O}_{4}$ was prepared and further conjugated with concanavalin A (a lectin protein) to produce GQD-ConA@ $\mathrm{Fe}_{3} \mathrm{O}_{4}$ nanocomposites. These were employed for both the recognition of HeLa cancer cells and release of drugs. To study their capability to preferentially recognize HeLa cancer cells over normal endothelial cells, an electrochemical method was employed. These nanocomposites were deposited on a Pt electrode, which allowed the assessment of the dynamic linear range $\left(5 \times 10^{2}\right.$ to $1 \times 10^{5}$ cells per $\left.\mathrm{mL}\right)$, and a detection limit of 273 cells per mL. Also, these nanocomposites could function as magnetically controlled nanocarriers for loading and releasing Dox. The in vitro results showed that the Dox concentration in HeLa cells was more than doubled in the presence of an external magnetic field due to the presence of $\mathrm{Fe}_{3} \mathrm{O}_{4}$ within the nanoplatform. The cytotoxicity in HeLa cells was $13 \%$ higher than normal cells because of the selective targeting by ConA. Thus, the GQD-ConA@ $\mathrm{Fe}_{3} \mathrm{O}_{4}$ nanocomposites are a promising theranostic platform for cancer cell detection and targeted Dox release. ${ }^{268}$ 


\subsection{Gene therapy}

Gene therapy is considered a promising treatment for many diseases, but some barriers limit its clinical translation. Gene therapy involves the delivery of nucleic acid-based therapeutic agents into cells to modify the expression levels of various genes. Depending on the type of agent they can be activated in the cell nucleus or the cytoplasm. Genes, their expression, translation and transcription are a complicated system and their manipulations may cause serious implications on the functions of biological systems. Moreover, transferring a desired gene to the target properly without any damage to the gene is another limitation. Delivering other nucleic acids will also affect gene regulations in a complex manner. Therefore, gene delivery systems should be multifunctional besides being specific for certain targets and protective for their contents. Accordingly, GQDs and CQDs present a suitable function for this purpose due to their physiochemical properties. Gene delivery systems based on GQDs and CQDs should interact properly with nucleic acids, precisely deliver them to targeted cells, protect the nucleic acids from environmental conditions, not disrupt gene regulatory systems or other physiological process and cause proper gene expression or regulation. The parameters involved in efficient gene delivery are discussed below. ${ }^{269}$

The success of gene therapy largely relies on the effectiveness of safe and efficient gene delivery into the target cell without any damage to the structure of the nucleic acids. A review of the literature from 2004 to date shows that the use of carbon-based nanomaterials for in vitro and in vivo gene delivery has steadily increased. ${ }^{270,271}$ Among them, O-dots can deliver numerous types of therapeutic nucleic acids in order to modulate the gene expression profile of cells. Two main approaches are used to carry out this goal. In the first approach, transcriptionally proficient DNA is delivered into cells to replace a copy of a defective or missing gene, and thus make up for the deficiency of the encoded protein. In the second approach, various therapeutic nucleic acids are used to regulate gene expression at different levels, which can be antisense oligonucleotides (AONs), small interfering RNA (siRNA), short hairpin RNA (shRNA) or microRNA (miRNA). These are used to inhibit or destroy the expression of unwanted genes. ${ }^{272}$

An efficient carrier for gene delivery should be capable of binding to nucleic acids properly and protecting them from environmental hazard conditions. These nanocarriers should be able to preserve the genetic materials during transportation, overcome extracellular and intracellular barriers, and deliver the functional form of the nucleic acid into the target cells in an intact manner. ${ }^{273}$

Several important physiochemical parameters, including the surface charge, size, shape and surface chemistry of the carrier should be optimized to produce a nano-gene delivery system (Fig. 17). By adjusting these factors, the fate of nanocarriers inside the body can be rationally tailored, and accordingly the final success of gene therapy can be realized. ${ }^{274}$

\subsubsection{Physiochemical parameters in gene therapy nanocarriers}

7.3.1.1. Surface charge. It is important to study the stability of nanocarriers, their cellular uptake mechanism, and their possible cytotoxicity. The overall surface charge probably has the most important role in facilitating these processes properly. ${ }^{275}$ Because nucleic acids (DNA, RNA, and ODN) possess an overall negative charge due to the phosphate groups in their backbone, they cannot pass across cellular membranes by simple diffusion, and therefore they require carrier systems, which can form complexes, leading to improved transportation across biological membranes. These polyplexes overcome the electrostatic repulsion between nucleic acids and the negatively charged cell membrane, and thereby expedite their internalization into cells. ${ }^{273}$ One simple approach to attach genetic materials to carriers is based on the electrostatic interactions between positively charged nanocarriers and nucleotides. ${ }^{276}$

Both charge polarity and charge density can affect the cellular uptake of nanoparticles, while simultaneously affecting their cytotoxicity. Positive surface charges combined with neutral or negative charges can improve the cellular uptake of nanoparticles allowing adhesion to the cell membrane in both phagocytes and non-phagocytic cell lines. ${ }^{277}$

Carriers with the highest positive charge density can be taken up to a higher extent at a faster rate. ${ }^{275}$ Additionally, the surface charge of nanocarriers determines the mechanism of cellular endocytosis. Nanocarriers can be internalized in cells via two pathways, i.e. clathrin-mediated endocytosis and caveolae-mediated pathways. Positively charged carriers use the first pathway, while carriers with a negative surface charge can be taken up through the second one. ${ }^{278}$

In comparison with the effects of negatively or neutral charged nanoparticles, positively charged nanoparticles exhibit greater overall cytotoxicity. ${ }^{277}$ Positively charged species can increase the $\mathrm{Ca}^{2+}$ influx into cells, and thereby inhibit their proliferation, disturb the structure of the lipid bilayer and its fluidity. ${ }^{279}$ Nanoparticles with a higher surface charge are more resistant to the formation of aggregates due to their strong selfrepulsion. ${ }^{274}$ It is clear that the surface charge of the carriers will be affected by gene loading. Due to the electrostatic interaction between the positively charged carrier and negatively charged nucleic acids, the surface charge of the complex will be partly neutralized. ${ }^{280}$ The carrier/DNA ratio (known as the $\mathrm{N}: \mathrm{P}$ ratio) usually imposes a complex charge, which increases to more positive values with an increase in the $\mathrm{N}: \mathrm{P}$ ratio. However, at a lower $\mathrm{N}: \mathrm{P}$ ratio, the formed complex may be incapable of effectively interacting with the cell membrane. ${ }^{281}$

7.3.1.2. Size. Another necessary factor in gene delivery is the size of nanocarriers, which can noticeably influence their colloidal stability, cellular uptake, transfection efficiency, residence in circulation, and the rate of clearance. ${ }^{274}$ In vitro experimental results revealed that nanoparticles are taken up by cells more efficiently as their size decreases. ${ }^{282}$ Nanoparticles with a size of around $20 \mathrm{~nm}$ can be taken up via cells in an endocytosis-independent manner. Longer circulation times in vivo can be achieved by the administration of nanoparticles 


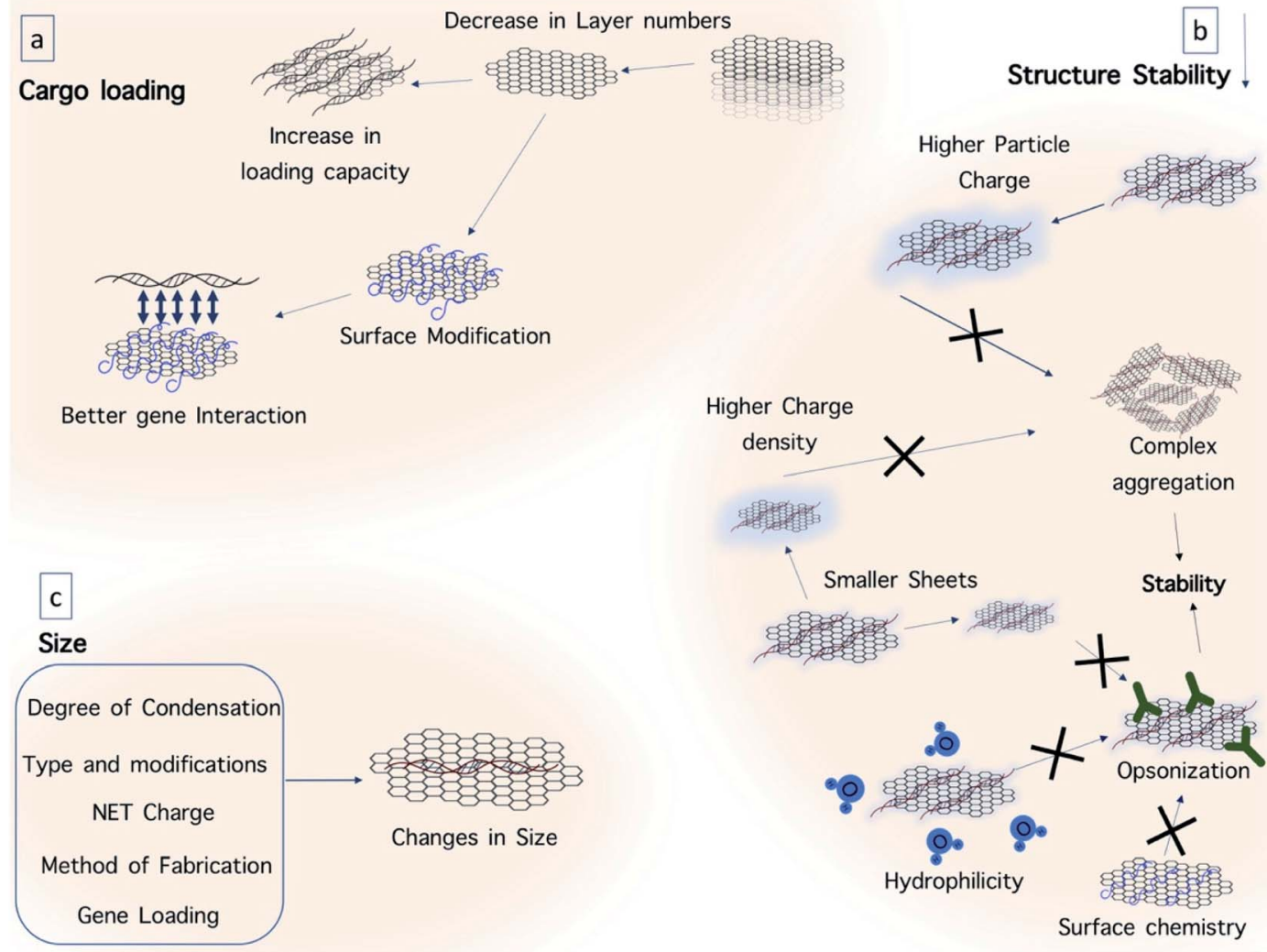

d

Electrical Charge

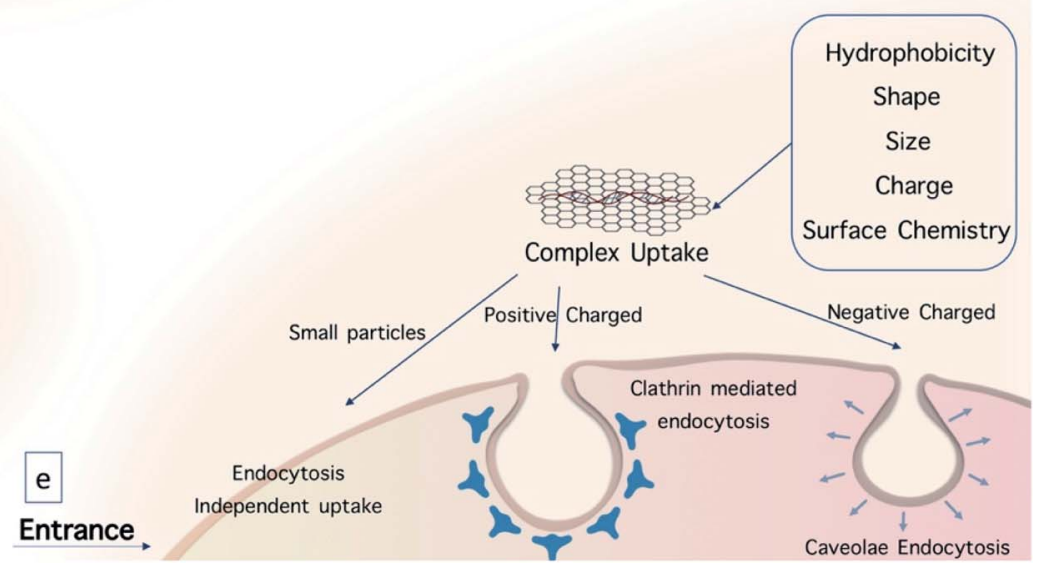

Fig. 17 Gene therapy based on graphene quantum dots. Several parameters should be considered in gene delivery and gene therapy. (a) Number of layers and surface modification of GQDs play a key role in the loading capacity of the particles. (b) Size, electrical charge, hydrophobicity and surface chemistry of GQDs are important parameters in their structural stability and longer circulation. (c) Electrical charge, condensation, nature and amount of cargo, synthetic method and modifications would change the size of the particles, which leads to altered performance. (d) Nucleic acid loading and changes in size determine the net charge of the particles. (e) Different properties of the GQD complex determine the route of its entrance into cells.

with a size smaller than $200 \mathrm{~nm}$. Smaller nanoparticles demonstrate lower clearance and better delivery to tumor tissues in comparison with larger nanoparticles, while they are not accumulated in other organs, such as the lungs, liver and spleen at this level. On the other hand, particles larger than $200 \mathrm{~nm}$ are rapidly removed by the liver, spleen and lungs. ${ }^{282} \mathrm{In}$ the case of O-dots, size also has an important effect on amphiphilicity, and thus smaller sheets are more hydrophilic due to their higher edge-to-area ratio. Moreover, smaller graphene sheets show better colloidal stability because of their higher charge density. ${ }^{283}$ The layer number is another parameter that should be considered in the design of O-dots since this governs the specific surface area and bending stiffness. ${ }^{284}$ By decreasing the number of layers to one or more, the loading capacity of the cargo will be improved. The size of the carrier can also be strongly affected by the binding of nucleic acids. ${ }^{285}$ 
The electrostatic interaction between the carrier and nucleic acids leads to condensation or compaction of the genetic material. The degree of condensation affects the size variability. However, the size of the complex can be altered by changing the structure of the cationic carrier, the $\mathrm{N}: \mathrm{P}$ ratio and also the fabrication method. For instance, by increasing the $\mathrm{N}: \mathrm{P}$ ratio during complex formation, the complex size will show a decreasing trend due to more efficient condensation of the loaded nucleotides. ${ }^{286}$ Therefore, the $\mathrm{N}: \mathrm{P}$ ratio during complexation should be precisely optimized before transfection. ${ }^{287}$

7.3.1.3. Shape. The shape of nanocarriers also governs their transportation across biological barriers and their fate inside cells. $^{282}$ Spherical-shaped nanoparticles smaller than $100 \mathrm{~nm}$ are taken up more efficiently in contrast to rod-shaped nanoparticles, and therefore lower cell uptake occurs as the aspect ratio of nanorods increases.

7.3.1.4. Surface chemistry. It is known that the surface chemistry of nanocarriers affects their biocompatibility, circulation within the body, and their interaction with the cells. The blood half-life of nanocarriers is governed by their surface hydrophobicity. As the hydrophobicity of the surface increases, more proteins including opsonin bind to the surface and complexes would be taken up more rapidly, which subsequently leads to a shorter circulation time. ${ }^{277}$ Nanoparticles with a hydrophobic surface diffuse into cells and tissue more quickly than that with a hydrophilic surface due to the hydrophobic nature of the cell membrane. ${ }^{282}$ Many methods have been employed to modify the surface of nanoplatforms to improve the biocompatibility, circulation time, cellular uptake and thereby the transfection efficiency of nanocarriers. ${ }^{274}$ Since Odots are intrinsically non-cationic, different modification approaches such as modification with PEI, chitosan, peptides, dendrimers, and PEG derivatives have been explored to provide cationic surfaces for a better interaction with anionic nucleic acids.

7.3.2. O-dots in gene delivery. Ghafari and coworkers prepared peptide-modified GQDs with two different (green and red) emission colors, and tested them for gene delivery and nuclear targeting applications and cellular tracking. ${ }^{288}$ The GQDs were physically linked to the MPG-2H1 chimeric peptide, which contained three different amino acid sequence motifs. These motifs were designed to carry out three different functions, including, DNA packaging, endosomal escape, and nuclear targeting. These modified O-dots facilitated cell tracking and showed improved transfection of the luciferase plasmid into HEK 293 embryonic kidney cells.

In another study, researchers prepared a multifunctional nanocomposite containing poly(L-lactide) (PLA), polyethylene glycol (PEG) and GQDs (f-GQDs) for the rapid imaging analysis of intracellular microRNAs (miRNAs), and combined gene delivery for improved therapeutic effects. The functionalization of GQDs with PEG and PLA enabled the f-GQDs to be stable in the physiological environment and also to have constant photoluminescence over a broad $\mathrm{pH}$ range, which is required for cell imaging. The f-GQDs possessed outstanding biocompatibility, low cytotoxicity, and protection of the cargo. The f-GQDs could efficiently transport a miRNA probe for intracellular miRNA imaging into HeLa cells. The large surface area of the GQDs could concurrently bind to the agents targeting miRNA21 , which led to the inhibition of cancer cell growth. ${ }^{289}$

Carbon quantum dots can also be applied as gene delivery platforms, especially N-doped CQDs, which generally possess a high quantum yield. For instance, N-CQDs fabricated using citric acid and tryptophan (Trp) were found to have good biocompatibility and capability to be functionalized with polyethyleneimine in order to deliver siRNA against the antiapoptotic protein survivin into human gastric cancer cells. The siRNACQDs@PEI complex was rapidly taken up by cells and down-regulated the expression level of survivin mRNA. Flow cytometry showed that the nanocomposites could cause a mixture of early apoptosis, late apoptosis, and necrosis in the target cells. ${ }^{290}$

Furthermore, fluorescent CQDs were attached to siRNA against polo-like kinase-1 (Plk1) to strongly down regulate the expression of Plk1, an essential regulator of mitosis. The required amount of FCNs was only $\sim 1 / 30$ of that of gold nanoparticles to deliver an equal amount of siRNA. They showed strong antitumor effects in vitro and in vivo by gene silencing, which was even greater than siRNA transfection by the positive control Lipo2000/siPlk1. ${ }^{291}$

Kim et al. $(2017)^{292}$ reported the use of CQDs as a functional siRNA delivery system for effective gene knockdown in vitro and in vivo. They showed that CQDs increased the uptake of siRNA by tumor cells via endocytosis, accompanied by low cytotoxicity and interesting effects on the immune system. Fluorescence images verified the localization of the CQDs in the cytoplasm and release of siRNA within $12 \mathrm{~h}$. The functional siRNA delivery system mediated by CQDs-PEI was used in an in vivo mouse model, with good gene knockdown efficacy and protection of the siRNA from degradation in vivo. Recently, Yang et al. $(2017)^{293}$ reported a simple one-step hydrothermal carbonization procedure for the synthesis of positively charged CQDS using PEI and FA as carbon sources. The cytotoxicity experiments confirmed that the toxic effects of PEI were lower in the presence of CQDs, and the composite could be useful as a probe for the selective imaging of folate receptor (FR)-positive cancer cells compared to normal cells. The surface of CQD/pDNA had a positive charge, which facilitated the interaction with the weak negative charges of the cell membrane, suggesting that the surface of CQDs could efficiently capture pDNA molecules. To study gene expression in vitro, they used transfection with CQDs, and pDNA encoding enhanced green fluorescent protein (EGFP) in 293T and HeLa cells. These results showed that the positively-charged CQDs could efficiently transfect cells and could be useful for gene therapy.

Hyaluronic acid (HA) is a natural, biodegradable, biocompatible polymer that specifically recognizes and binds to CD44, a receptor expressed on the surface of cells, including tumor cells. HA was used in the construction of an O-dot-based nanovehicle, which can be useful in tumor therapy. ${ }^{294}$ Wang et al. ${ }^{295}$ described the synthesis of CQD particles, in which HA was utilized as the carbon source and PEI as a passivation agent using a simple microwave (MW)-assisted pyrolysis procedure. In 
this study, the presence of HA improved the biocompatibility of the CQDs and the cationic amine groups on PEI helped DNA condensation and triggered endosomal release once internalized. Gel retardation and ethidium bromide (EtBr) exclusion assays suggested that the minimum $\mathrm{C} / \mathrm{P}$ ratio required to inhibit DNA migration was equal to 1 , while analysis of the particle size and zeta potential suggested that a $\mathrm{C} / \mathrm{P}$ ratio above 6 was the most suitable for transfection. In comparison to PEI, these materials demonstrated lower cytotoxicity and up to 50fold higher transfection efficiency in the presence of $10 \%$ serum. In vitro experiments using BSA protein binding, flow cytometry, and confocal microscopy also showed effective gene delivery. The fluorescent properties of these particles allowed the monitoring of the DNA internalization process, while an HA competition assay showed the specific ability of HA-QDs to identify cells overexpressing CD44. Different aspects of the use of GQD in gene delivery is shown in Fig. 17.

\subsection{Bioimaging applications}

Other major application of GQDs and CQDs is bio-imaging. This application is based on their photoluminescence properties, ${ }^{296,297}$ although they can be hybridized with other particles for use in other imaging modalities. ${ }^{298-300}$ Bioimaging can both provide basic knowledge about biological systems and used in diagnostic methods. Bioimaging is one of the pivots of theranostics, and since GQDs and CQDs are suitable cargo carriers and demonstrate other therapeutic activities including photothermal therapy and photodynamic therapy, showing bioimaging capabilities, they are promising theranostic agents.

Luminescent nanoparticles have been widely used for bioimaging applications and visualizing living cells. However, their potential toxicity is still a problem, which may limit the bio-imaging application of many luminescent nanoparticles. Odots possess low toxicity and good biocompatibility, coupled with stable PL, and therefore they are ideal candidates for both in vitro and in vivo bio-imaging. In this section we discuss the recent progress in bio-imaging applications both in vitro and in vivo. ${ }^{301}$

7.4.1. In vitro imaging. Many studies have reported the use of O-dots for in vitro bio-imaging. ${ }^{302-304}$ In several applications, QDs have been shown to be superior to organic dyes and fluorescent proteins due to their enhanced brightness, photostability, imaging sensitivity, and good resistance to metabolic degradation. ${ }^{305}$ Semiconductor QDs such as CdSe with a coreshell structure (e.g. CdSe/ZnS) have been used for in vivo/in vitro bio-imaging. ${ }^{306}$ However, the toxicity of cadmium-containing QDs is a concern due to their long-term accumulation in tissues and organs. Thus, to compare the ability of CQDs in bioimaging with classical semiconductor QDs (CdSe/ZnS), researchers used CQDs prepared via nitric acid treatment of carbon soot and then high-temperature treatment with PEG1500N to attach oligomeric PEG. They found that the CQDs were more biocompatible and performed equally well in bioimaging in comparison with CdSe/ZnS QDs. Based on fluorescence images of CQDs and CdSe/ZnS commercial QDs under high dilution conditions, the CQDs showed good and non- blinking fluorescence properties (QY of 20\% at an excitation wavelength of $440 \mathrm{~nm}$ ). One advantage of CQDs is that they have a smaller size compared to the CdSe/ZnS commercial QDs. The size of CQDs is less than $10 \mathrm{~nm}$, while that of commercial QDs is larger than $20 \mathrm{~nm}$. The smaller sizes of CQDs offer the opportunity to image small biological structures and also a lower volume will be needed for in vivo injections. ${ }^{305}$ Cao et al. fabricated CQDs with strong luminescence upon two-photon excitation in the NIR region, and used poly(propionyl ethylenimineco-ethylenimine) for surface modification. They confirmed that the two-photon absorption cross-section of the purified CQDS was comparable with semiconductor QDs. These CQDs were water-soluble with a size less than $5 \mathrm{~nm}$. The luminescence intensities of the CQDs depended on the square of the power of the excitation laser (pulsed infrared laser), confirming twophoton excitation. After incubation of human breast cancer cells (MCF-7) with the CQDs and excitation with $800 \mathrm{~nm}$ laser pulses, the cells could be visualized under a 2-photon fluorescence microscope. The CQDs labeled the cell membrane and the cytoplasm without localizing in the nucleus.

The accumulation of CQDs inside cells can be improved if they are conjugated with TAT, a human immune deficiency virus-derived cell-penetrating peptide. TAT-conjugated CQDS were able to penetrate the cell nucleus. ${ }^{307}$ The surface passivation of CQDs for bio-imaging can affect their optical properties. For example, CQDs were prepared by microwave treatment of maltose, and passivated with dilute $\mathrm{NaOH}$ solution. The $\mathrm{NaOH}$ solution produced $-\mathrm{OH}$ groups on the $\mathrm{sp}^{2}$ hybridized carbon atoms, which increased the QY of the CQDs. It was found that these CQDs were taken up by the cells and produced a green fluorescence emission, while the cell viability was unaffected even after $24 \mathrm{~h}$ incubation. ${ }^{308}$

In other experiments, ${ }^{309}$ surface passivation could change both the fluorescence intensity and the emission wavelength. The presence of $-\mathrm{OH}$ groups can create hydrophilic CQDs, which are beneficial for bio-imaging applications. For example, CQDs with a particle size of $2-6 \mathrm{~nm}$ and a QY of $3 \%$ were produced by refluxing soot with nitric acid. The oxidation of the soot with nitric acid formed both $-\mathrm{OH}$ and $-\mathrm{CO}_{2} \mathrm{H}$ groups on the CQDs, making them negatively charged and hydrophilic. The in vitro experiments showed that the CQDs with no further passivation were taken up into the cells and released fluorescence. Under UV irradiation, the cells emitted blue-green fluorescence, and under blue excitation, a yellow emission was observed in the sample, whereas the sample without CQDs was colorless under the abovementioned excitations. ${ }^{\mathbf{3 1 0}}$

When CQDs are purified by dialysis, the dialysis tube pore size or its molecular weight cut-off (MWCO) has a substantial influence on the emission characteristics of the CQD solution. For instance, in one study, ${ }^{311}$ the effect of the dialysis membrane molecular weight cut-off varying from 3500 to 14000 Da was examined, and it was found that the excitation wavelength for the oxidized CQDs changed for the different pore sizes, which emitted at different wavelengths in the range of 360 to $460 \mathrm{~nm}$. The CQDs from membranes with MWCO > $3500 \mathrm{Da}$ (up to $7000 \mathrm{Da}$ ) had similar emission intensities, while that collected by the membranes with an MWCO of 7000- 
14000 Da had a reduced emission intensity. The CQDs that were collected by the membrane with an MWCO of less than 3500 Da had a low QY, and were contaminated with salts. The CQDs obtained from dialysis with an MWCO of 3500-7000 Da had the best optical properties. ${ }^{311}$

Furthermore, oxidation has a strong effect on the PL features of CQDs. For instance, in chemically produced GO thin films in which isolated $\mathrm{sp}^{2}$ clusters were formed in an $\mathrm{sp}^{3}$ matrix of carbon-oxygen bonds, greater localization of the electron-hole pairs and better radiative recombination were observed, and the PL intensity was influenced by the reduction treatment. The reduction process altered the small $\mathrm{sp}^{2}$ clusters, and therefore, altered the PL intensity. The PL in graphene can be adjusted by engineering its $\mathrm{sp}^{2}$ clusters by controlled oxidation. ${ }^{312}$ Similar results were found by $\mathrm{Hu}$ et al., ${ }^{313}$ who reported that the addition of oxidized branched polyethylene imine (BPEI) improved the band gap by modifying the oxygen-carbon matrix of the CQDs. The CQDs prepared through oxidation and hydrothermal treatment using BPEI, with a diameter of 3-4 $\mathrm{nm}$ could provide a QY as high as $54.3 \%$. Incubation of the CQDs with MCF-7 cells and excitation at $405 \mathrm{~nm}$ showed luminescence inside the cells dispersed throughout the cytoplasm. The PL released from the CQDs did not decrease during 30 min excitation, indicating low photobleaching and good photostability. ${ }^{313}$

Larger carbon nanoparticles can also show fluorescence, for instance CQDs with a mean particle size of $70 \mathrm{~nm}$ can emit fluorescence and could be utilized for bio-imaging. ${ }^{\mathbf{3 1 4}}$ The fluorescent CQDs were prepared via hydrothermal treatment of cocoon silk. During the hydrothermal treatment, the silk fibers were first cut into shorter ones, and then by heating at $200{ }^{\circ} \mathrm{C}$ were converted to asymmetrical polymeric fragments, and lastly to larger CQDs. The human cervical cancer (HeLa) cells incubated with the CQDs showed intracellular fluorescence. Confocal laser scanning microscopy images showed fluorescence in the perinuclear areas of the cytosol, confirming the effective uptake of the CQDs into the cells. These CQDs were used for in vivo tissue imaging of human tumor xenografts (MCF-7) in nude mice. The fluorescence emission observed in the tumor confirmed the permeability of the CQDs, while they showed low photobleaching and no blinking. ${ }^{\mathbf{3 1 4}}$

Another valuable feature of CQDs for bio-imaging applications is their photostability. For example, the changes in PL intensity of the CQDs inside COS-7 cells were studied after incubation for $24 \mathrm{~h}$. The COS-7 cells became bright, which revealed that the CQDs entered the cells, and the CQDs were able to label both the cell membrane and the cytoplasm of COS7 cells. There was no apparent decrease upon continuous excitation for $10 \mathrm{~min}$. This result was ascribed to the low photobleaching and high photostability of the CQDs. ${ }^{315}$

CQDs generally possess low cytotoxicity, even if they have been obtained from noxious components. For instance, CQDs prepared from halophenols (a group of industrial pollutants) were applied as fluorescent labeling agents. After incubation of the CQDs with HeLa cells and excitation with a $405 \mathrm{~nm}$ laser, the cell membrane and perinuclear cytoplasm showed fluorescence, while the signal inside the cell nucleus was weak. No morphological damage was observed in the cells after incubation with the CQDs, which signified their low cytotoxicity. ${ }^{316}$ Plants, fruits and food products such as orange juice, ${ }^{317}$ apple juice, ${ }^{318}$ gelatin, ${ }^{319}$ milk, ${ }^{320}$ soy milk, ${ }^{321}$ honey, ${ }^{322}$ and egg.117 have all been used as a carbon source for the preparation of CQDs. It is thought that the use of natural products and foodstuffs may lead to lower toxicity of the resultant CQDs for biomedical applications. Mehta et al. ${ }^{323}$ used Saccharum officinarum juice as the carbon source for the fabrication of CQDs. These CQDs were used for cell imaging in E. coli (bacteria) and $S$. cerevisiae (yeast). The laser confocal fluorescence microscopic images showed that different colors (red, green and blue) could be observed in the labeled bacteria and yeast cells using $40 \mathrm{mg}$ $\mathrm{mL}^{-1}$ of CQDs. In the confocal images of $E$. coli, the CQDs were well distributed in the membrane and cytoplasm. The CQDS were taken up by the yeast cells via an endocytosis mechanism. The CQDs were located in the yeast nucleus, but the yeast cells maintained $>90 \%$ viability. ${ }^{323}$ Orange juice was used to make CQDs via a hydrothermal process, exhibiting a QY of $26 \%$. These CQDs were used as imaging probes in human osteosarcoma (MG-63) cells, where they accumulated in the cytoplasm but not the nucleus. The PL intensity did not decay over a continuous excitation period. ${ }^{324}$

$\mathrm{N}$-doped CQDs can be prepared by doping with various nitrogen-containing molecules such as diamine to increase their QY for bio-imaging probes. N-doping resulted in a QY as high as $36.3 \%$. Fluorescent images ( $355 \mathrm{~nm}$ excitation) of HeLa cells showed that their cytoplasm was brighter than that with non-doped CQDs, indicating that labeling with N-doped CQDs can be performed at lower doses. Continuous UV exposure caused the fluorescence of the non-doped CQDs to be bleached after $15 \mathrm{~min}$, while the fluorescence of the N-doped CQDs was still bright after the same period. ${ }^{325}$

Stem cells are fundamental to the understanding of embryonic development and tissue regeneration because they can give rise to several progenitor cells, which can in turn differentiate into cells of different tissue types. ${ }^{326}$ Due to the generally quiescent state of stem cells, their labeling processes face a substantial challenge. ${ }^{327}$ Bright yellow fluorescent GQDs ${ }^{328}$ showed direct and easy uptake into stem cells without affecting their viability, proliferation or differentiation capacity. This was the first use of stabilizer-free graphene as a fluorescent label in long-term stem cell imaging. Three different types of stem cells, namely neurosphere cells (NSCs), pancreatic progenitor cells (PPCs), and cardiac progenitor cells (CPCs) were incubated with GQDs at a concentration of $25 \mathrm{mg} \mathrm{mL}{ }^{-1}$ for $24 \mathrm{~h}$ at $37{ }^{\circ} \mathrm{C}$. The confocal fluorescent images showed that the GQDs were localized in the cytoplasm of the stem cells. The methyl thiazolyldiphenyl tetrazolium bromide (MTT) assay verified the low cytotoxicity of GQDs for stem cell imaging. In another study, the uptake mechanism and biocompatibility of GQDs interacting with human neural stem cells (hNSCs) were examined. ${ }^{329}$ TEM images proved that the GQDs were internalized into hNSCs via an endocytosis mechanism and were localized in the cytoplasm. The researchers reported that GQDs did not influence the selfrenewal capacity of hNSCs. Single cells separated from GQDlabeled hNSCs still formed large neurospheres, and the cells expressed specific markers for hNSCs. In addition, no changes 
were detected in the viability, proliferation, metabolic activity or differentiation potential of NSCs after treatment with GQDs. After 14 days of differentiation, no differences were discovered in the hNSC proliferation rate between the control group and the $25 \mu \mathrm{g} \mathrm{mL}{ }^{-1}$ GQDs treatment group. The cells from both groups exhibited an elongated cell morphology with neurite outgrowth, resulting in the formation of an interconnected neuronal network. Expression of glial fibrillary acidic protein (GFAP) and neuron-specific class III beta-tubulin (Tuj1) showed that the hNSCs could differentiate into glial cells and neurons, respectively. In addition to the bio-imaging of stem cells, other studies of GQD-based imaging have focused on tumor cells. Numerous studies have confirmed that fluorescent GQDs are taken up by tumor cells. In 2014, Yang et al. reported the use of B-GQDs for the imaging of HeLa cells. ${ }^{330} \mathrm{Zhu}$ et al. cultured human osteosarcoma (MG-63) cells with solvothermally fabricated GQDs, which showed two-color imaging using different excitation wavelengths. ${ }^{331}$ Peng et al. incubated green luminescent GQDs with the T47D human breast cancer cell line and stained their nucleus with DAPI. ${ }^{332}$ The images included a phase contrast, where the nuclei were stained blue with DAPI, and high contrast green fluorescent GQDs were observed in the perinuclear region in the triple over-layered images.

Dong et al. ${ }^{333}$ incubated MCF-7 human breast cancer cells with GQDs synthesized via acidic oxidation, and a bright green emission could be detected using confocal laser scanning microscopy with excitation at $488 \mathrm{~nm}$. The section analysis of single MCF-7 cells showed that GQDs not only labeled the cell membrane and the cytoplasm, but also the nucleus. This was the first time that luminescent carbon nanomaterials were shown to tag the cell nucleus. Moreover, Hela cells, ${ }^{334}$ A-549 cells, ${ }^{335}$ macrophages and hepatocellular cells ${ }^{335}$ have all been labeled with fluorescent GQDs by different groups. All these studies reported the low cytotoxicity and biocompatibility of GQDs using the MTT assay, and hence they could be used for bio-imaging. Fluorescent GQDs functionalized with folic acid (FA) were introduced as highly selective and specific tumor cell imaging agents by Wang et al. ${ }^{336}$ The fluorescence of the GQDs in FA over-expressing HeLa cells was noticeably stronger than in A549 and HEK293A cells, which both express the FA receptor (FR) at low levels.

7.4.2. In vivo imaging. CQDs have also been used by several groups for in vivo imaging in living animals..$^{37-339}$ Due to their low toxicity, CQDs are a better choice for in vivo imaging than heavy metal-containing QDs. CQDs that are obtained from diverse carbon sources and are functionalized with suitable functional groups are capable of emission under excitation in both the UV and NIR regions of the electromagnetic spectrum. For in vivo optical imaging, it is better to use longer wavelengths since the photon penetration in tissue is higher and the background auto-fluorescence is lower. Although the fluorescence emission of CQDs is often weaker at longer wavelengths, imaging at these wavelengths is better to reduce the tissue autofluorescence background and leads to higher signal-to-noise ratios overall. For example, CQDs synthesized via the oxidization of carbon nanotubes with a size of 3-4 $\mathrm{nm}$ were used for in vivo imaging in mice. After excitation with blue, green, yellow, orange, red, deep red and NIR light and segregating the green background auto-fluorescence, clear spots were observed in the fluorescence images with red or longer excitation wavelengths. In comparison to the images captured with blue and green excitation, excitation at $595 \mathrm{~nm}$ and higher showed the best signal-to-background ratio. ${ }^{340}$

CQDs have some advantages compared to other commercially available QDs for bio-imaging due to their high absorption coefficients. The higher absorptivity of CQDs can compensate for their lower fluorescence yield compared to heavy metal QDs. In one study, PEG surface-passivated CQDs were compared with CdSe/ZnS QDs. The PEG CQDs had better absorptivity and they also protected the dots for in vivo bioimaging. ${ }^{305}$

The NIR emission of CQDs that were functionalized with a hyper branched polymer increased compared to the CQDS coated with a linear polymer (PEG). In vivo experiments in a mouse model led to visualization of lymph nodes due to the fluorescent emission of the CQDs. ${ }^{341}$ CQDs can also be doped with inorganic salts (e.g. ZnS) to increase their fluorescence yield for in vivo imaging. Injection of CQDs doped with ZnS (CQDs-ZnS) into mice (compared to the non-doped CQDs) showed a brighter emission. Green fluorescent CQD-ZnS was injected intradermally into the footpad and then transported along the leg via the lymphatic vessels. The migration of CQDZnS was slow in comparison to conventional semiconductor QDs. One reason was that the size of the CQDs-ZnS was smaller, while another reason could be due to the PEG functionalization. These two properties would result in decreased interactions with lymphatic cells. ${ }^{342}$

GQDs can also be exploited for in vivo imaging. Passivated nanographene sheet particles with attached PEG (NGS-PEG) with an average size of $30 \mathrm{~nm}$ were prepared by Yang et al. and administered into mice after tagging with the commonly used NIR fluorescent dye Cy7. In vivo fluorescence imaging showed the high tumor uptake of the NGS in several tumor xenograft mouse models. ${ }^{343}$ The effectiveness of GQDs fabri-

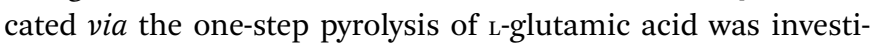
gated for in vivo imaging in mice by Wu et al. ${ }^{344}$ After injecting the GQDs intradermally into the back of nude mice and intramuscularly into their right back leg, fluorescence images of the mice using different excitation and emission filters were captured. The detectable fluorescence from the intramuscular injection site in the leg was higher with longer excitation and emission wavelengths because of the better tissue penetration of light. Ge et al. examined in vivo fluorescence imaging using red fluorescent GQDs prepared via a hydrothermal method and modified with polythiophene derivatives. ${ }^{345}$ After injection of the GQD aqueous solution into the back of a nude mouse, fluorescent spots were observed against the background signal of the mouse skin. The fluorescent spots remained visible up to 1 week after injection.

\subsection{Phototherapy}

Surgery, radiotherapy and chemotherapy are the most common methods for the treatment of cancer; however, these methods 
exhibit undesirable side effects on other healthy tissues of the body. Therefore, many novel treatment methods have been developed to reduce the side effects of cancer therapy. ${ }^{346}$ In recent years, several methods based on the therapeutic use of light, such as photothermal therapy (PTT) and photodynamic therapy (PDT), have attracted attention for the treatment of cancer. PPT relies on the generation of heat from light absorption, while PDT is based on the photochemical generation of reactive oxygen species to destroy tumors. ${ }^{346,347}$

Phototherapy is one of the independent therapeutic functions of GQDs and CQDs in synergism with light irradiation. The therapeutic function of phototherapy is based on the formation of lesions and causing damage to tissues. Accordingly, for more efficient function, besides the sonosensitizing properties of the material, targeting abilities are required for specialized therapy and reducing side effects, which can be provided by GQDs and CQDs due to their physiochemical properties. $^{346}$

7.5.1. Mechanism of PDT. Photodynamic therapy is a promising method for the treatment of human diseases, including several disorders of the skin, infections, and also cancer. PDT involves the application of a photosensitizer (PS) or a photoactive dye, and a particular wavelength of light tailored to the PS absorption spectrum (Fig. 18). PDT is considered noninvasive, selective and can be combined with other therapies. ${ }^{348}$ When PSs are exposed to a specific wavelength of light, they can generate a reactive form of molecular oxygen (singlet oxygen, ${ }^{1} \mathrm{O}_{2}$ ) with the ability to kill surrounding cells. This technique can be used solely or in combination with surgery, chemotherapy and ionizing radiation. ${ }^{349}$ Various PSs such as porphyrins and phthalocyanines have been shown to carry out simultaneous cancer imaging and tumor destruction, and some of these PSs have been approved for clinical applications. ${ }^{350}$ PDT is used in the treatment of different cancers such as skin cancer, liver tumors, esophageal cancer and malignant gliomas. ${ }^{351-353}$
The PDT procedure consists of 3 important elements, PS, light, and ambient oxygen. PSs absorb energy from light, and then the oxygen molecules turn into singlet oxygen and other reactive oxygen species, which induce rapid cytotoxicity, causing apoptosis and necrosis. ${ }^{354}$ PSs are classified in two groups, i.e. porphyrins including other tetra pyrrole derivatives, and nonporphyrins. ${ }^{350}$

7.5.2. Mechanism of PTT. Photothermal therapy is based on the absorption of photons by a chromophore, which produces heat from optical energy to kill cancer cells. ${ }^{355}$ NIR lasers are often used for PTT in order to target the tumor either directly or interstitially through an optical fiber. This energy is turned into heat through optical absorption followed by nonradiative relaxation of the excited state. Consequently, selective photothermal absorbing materials or chromophores can be used in the treatment of advanced tumors with little damage to surrounding healthy tissues. The aim of PTT is to uniformly increase the temperature within the tumor up to $41{ }^{\circ} \mathrm{C}$ or higher. Liao et al. recently claimed that $41{ }^{\circ} \mathrm{C}$ can initiate the degradation of tumor cells. ${ }^{356}$

\subsection{O-dots in phototherapy}

The properties of O-dots include their chemical inertness, high water solubility, photostability, interplay between optoelectronic features and shape/size, fluorescence resonance energy transfer, high stability under physiological conditions, specific accumulation at target sites, facile surface functionalization, and high absorption coefficient due to their $\mathrm{sp}^{2}$ hybridized $\mathrm{C}-\mathrm{C}$ bonds. Thus, these features make them an attractive chromophore for phototherapy. ${ }^{345,357,358}$

One problem with many clinically employed PSs is that they are not specifically targeted to tumors, and consequently spatially controlled irradiation is utilized to target the site of treatment. Moreover, PS administration can cause ROS production in healthy cells, meaning that care needs to be taken

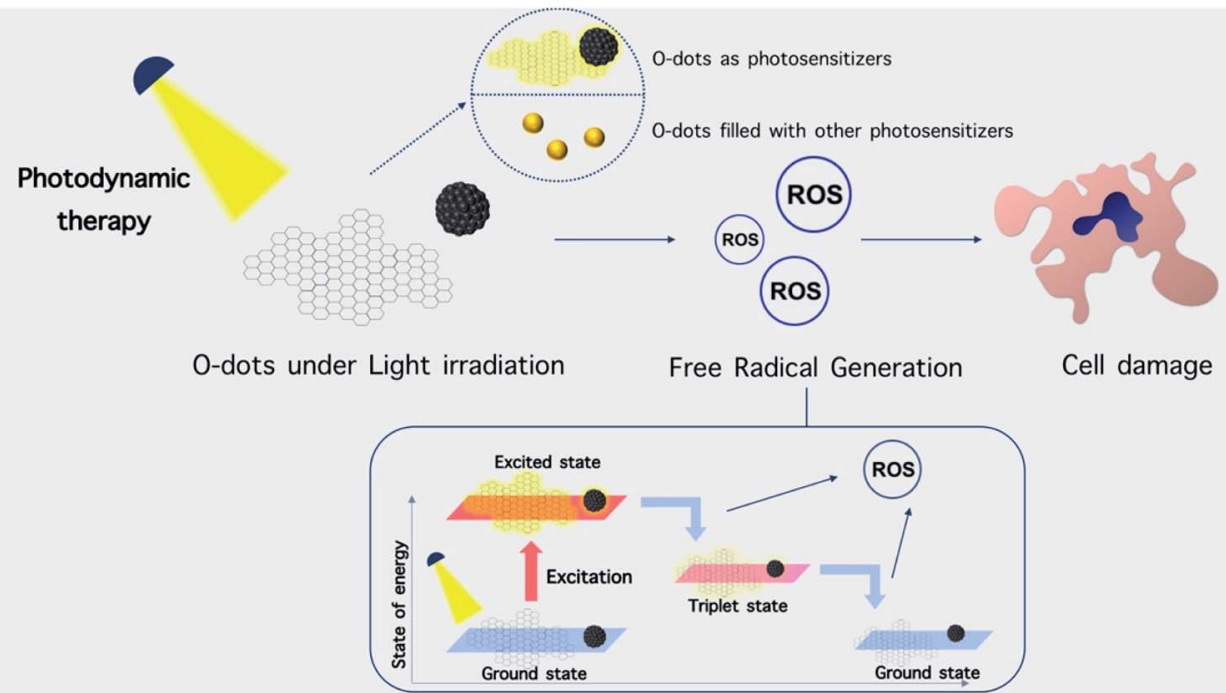

Fig. 18 Schematic illustration of phototherapy methods based on organic dots. O-dots can act as photosensitizers or they can be coated or filled with other photosensitizers. Excitation of $\mathrm{O}$-dots and their subsequent loss of energy to lower energy states lead to the generation of ROS. 
after phototherapies to prevent light exposure to lessen the risk of skin photosensitivity. ${ }^{345}$ The QY of ${ }^{1} \mathrm{O}_{2}$ production from NPs and quantum dots (QDs) alone are not as high as that from molecular PSs; however, O-dots can be applied as antennae to improve light harvesting and energy transfer to molecular PS owing to their high (size dependent ${ }^{345}$ ) light absorption crosssection. ${ }^{359}$ Furthermore, broad-wavelength excitable O-dots with tunable up-conversion and down-conversion properties can also be applied in PDT. ${ }^{360}$

The large number of mobile $\pi$-electrons in O-dots leads to comparatively strong electron-electron scattering and weak electron-electron interactions. It was predicted that the $\pi$ electrons in O-dots can act in a similar manner to the free electrons in metallic nano-clusters rather than that in inorganic semiconductor QDs. The limited QY of O-dots means that most of the absorbed light will be ultimately transformed into heat through several non-radiative relaxation pathways. This consideration suggests their potential use in PTT rather than PDT. ${ }^{361,362}$

7.6.1. O-dots in PDT. The concept of utilizing O-dots for cancer therapy dates back to 2015, when Liu et al. reported the synthesis of CQDs with high QYs as labeling agents for cells. The CQDs developed by Liu et al. exhibited significant dispersion and excellent optical properties such as high fluorescence and photostability, resistance to photobleaching, and facile coupling with biological species. They also reported that these structures can play a substantial role in PDT. ${ }^{363}$ In another study, Huang et al. reported that CQDs could be utilized as carriers for the PS drug Ce6, as well as the ability of these structures to generate ROS. ${ }^{364}$ As reported in their study, the ROS could be produced by the fluorescence resonance energy transfer phenomenon (FRET) from CQDs to Ce6. Zheng et al. doped CQDs with carbon nitride $\left(\mathrm{C}_{3} \mathrm{~N}_{4}\right)$, producing an increase in infrared absorption. ${ }^{365}$ They employed a polymer consisting of protoporphyrin and a tumor-targeting sequence for the functionalization of carbon nitride-doped CQDs. Zheng and his group used this approach to overcome tumor hypoxia and improve PDT, which is often not very effective in hypoxic tumors. Water splitting occurred, producing oxygen and hydrogen in vivo using irradiation from a $639 \mathrm{~nm}$ laser. The extra oxygen formed increased the yield of ${ }^{1} \mathrm{O}_{2}$ to improve PDT.

Carbon dots attached to a platinum-coordinated porphyrin formed a composite (CQDs@PtPor) through the electrostatic interaction between four platinated porphyrins and the negatively charged CQDs in a complex (PtPor). This was utilized as a theranostic agent for PDT cancer therapy. ${ }^{366}$ They claimed that the composite combined the optical properties of CQDs and the anticancer activity of porphyrin into a distinctive new structure.

Pheophytin is a type of Mg-free chlorophyll derivative and is also a natural product with low toxicity, which was used as a raw carbon source for the synthesis of CQDs with the assistance of a microwave method by Weng and co-workers. The hydrophobic CQDs exhibited high potential to emit NIR light, with an excitation peak at around $680 \mathrm{~nm}$, and high ${ }^{1} \mathrm{O}_{2}$ production with a quantum yield of 0.62 . The self-assembled CQDs from the precursor (DSPE-mPEG2000) showed efficient ${ }^{1} \mathrm{O}_{2}$ generation. This carbon dot assembly was found to be useful as a fluorescence imaging agent and also as a smart PDT agent. Thus, these nanostructures can serve as a new phototheranostic agent for cancer therapy. ${ }^{367}$

Meng and co-workers reported the synthesis of a novel multifunctional two-photon excited nano probe based on GQDs, GQD@ $\mathrm{MnO}_{2}$, with the capability for imaging glutathione (GSH) in biological systems and for improved PDT. GSH is a ubiquitous tri-peptide and the most common intracellular thiol, which has been reported to consume ${ }^{1} \mathrm{O}_{2}$. Thus, GSH can decrease the effectiveness of PDT, as confirmed in vitro by Meng et al. Their prepared nanoprobe exhibited high sensitivity and selectivity toward GSH because the reduction of $\mathrm{MnO}_{2}$ nanosheets could be carried out by intracellular GSH, which led to its overall consumption. Therefore, GSH removal could improve the efficacy of PDT by GQDs. Researchers reported that the PDT efficiency of the prepared nanoprobes was better than that of GQDs alone by incubating HeLa cells with GQDs or GQD@MnO $\mathrm{MnO}_{2}$ and irradiating with $560 \mathrm{~nm}$ light for $30 \mathrm{~min}$. In addition, they found that nitrogen-doped GQDs had a good twophoton absorption cross-section for enhanced PDT. Doping GQDs with $\mathrm{N}$ can cause widening the light adsorption peak and prolongation of the PL lifetime. NGQDs also exhibited a good PTT effect when compared with other doped materials. Recently, Wen et al. confirmed this hypothesis. ${ }^{367,368}$

The combination of GQDs and methylene blue (MB) to destroy pathogenic bacteria by enhancing light-mediated ${ }^{1} \mathrm{O}_{2}$ generation was reported by Monroe and co-workers. Moreover, their group also measured the ability of GQDS, MB and other GQD-MB combinations to kill MCF-7 breast cancer cells. They utilized the cell counting method to evaluate the cytotoxicity of GQDs, MB and a $1: 1$ GQD : MB mixture. The measurement of ${ }^{1} \mathrm{O}_{2}$ generation in the medium was then checked by means of singlet oxygen sensor, in which the dye $2^{\prime}, 7^{\prime}$-dichlorodihydrofluorescein diacetate $\left(\mathrm{H}_{2} \mathrm{DCFDA}\right)$ was used to measure the production of reactive oxygen species. Another dye, $\mathrm{H}_{2}$ DCFDA, was used to examine the total ROS generated during the process. Monroe and co-workers confirmed that GQDs and MB could be taken up into MCF-7 cells, and the most appropriate proportion for the combination was found to be $1: 1$, which led to superior cytotoxicity and the highest amount of ${ }^{1} \mathrm{O}_{2}$ and ROS. In general, they found that the effects on MCF-7 cells were not dependent on the GQD concentration or the intensity of light. ${ }^{369}$

7.6.2. O-dots in PTT. Bao et al. used co-doped CQDs with sulfur and nitrogen as in vivo theranostic agents in mouse models. ${ }^{370}$ They obtained high photon conversion efficiencies (>59\%), which were useful for photoluminescence and photoacoustic imaging. The dominant mechanism for the destruction of the tumor involved the accumulation of CQDs around the cancer cells via passive targeting with no active targeting species. A high photothermal conversion, good optical and photoacoustic performance, and renal excretion were observed for the co-doped CQDs. Nitrogen and oxygen co-doped CQDS (N-O-CQDs) with strong absorbance in the NIR region were prepared via a one-step molecular fusion route from the starting materials 1,3,6-trinitropyrene (TNP) and N-containing polymer branched polyethylene imine (BPEI) by Geng et al., as shown in Fig. 19. The black N-O-CQDs exhibited good photostability and 

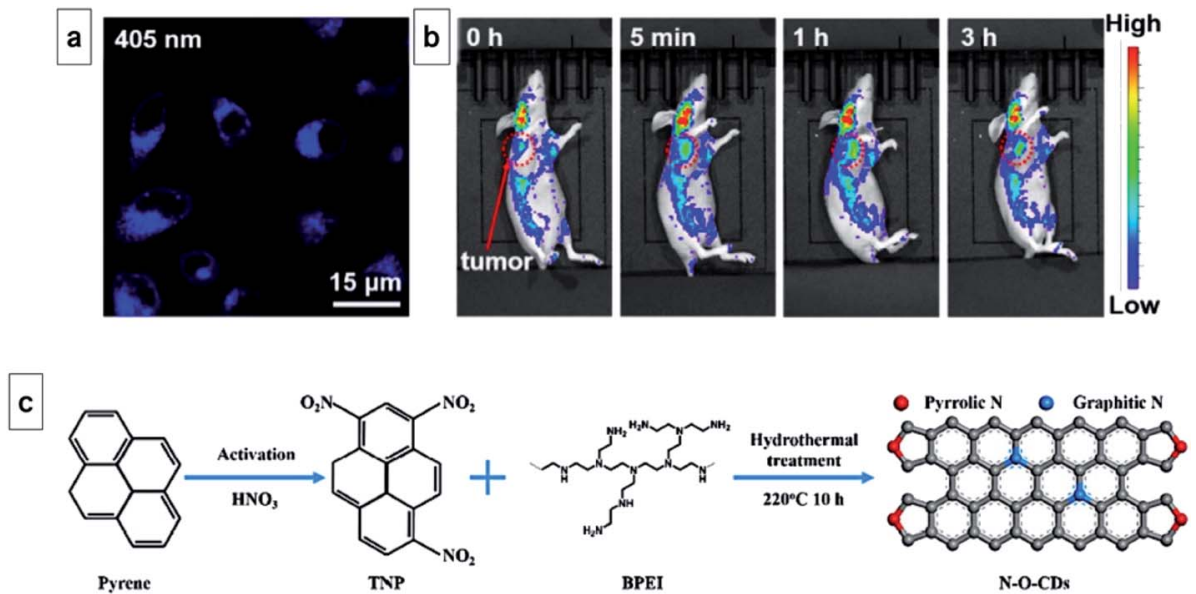

Fig. 19 (a) Confocal laser-scanning microscopy (CLSM) images of HeLa cells incubated with N-O-CDs (100 $\mu \mathrm{g} \mathrm{mL}{ }^{-1}$ ) excited by $405 \mathrm{~nm}$. (b). In vivo fluorescence imaging of the tumor site from a mouse intratumor injected with $\mathrm{N}-\mathrm{O}-\mathrm{CDs}$ at $0 \mathrm{~h}, 5 \mathrm{~min}, 1 \mathrm{~h}$, and $3 \mathrm{~h}$ post-treatment. The color bars represent the fluorescence intensity. Red circles indicate the position of the implanted tumor. (c) Synthetic procedure of $\mathrm{N}-\mathrm{O}-\mathrm{CQDs}$ (this figure has been reproduced from ref. 371 with permission from Elsevier, Copyright 2018).

biocompatibility, and could function not only as a new fluorescence imaging agent but also an excellent PTT agent in vivo. Moreover, the structure possessed good optical properties, bioimaging ability, photothermal effects, and therapeutic efficacy in vivo. The low power density used $\left(0.8 \mathrm{~W} \mathrm{~cm}^{-2}\right)$ and high PTT conversion efficiency $(\eta=38.3 \%)$ were claimed as advantages of $\mathrm{N}-\mathrm{O}-\mathrm{CQDS}$. Geng et al. claimed that this structure can act as a theranostic agent for fluorescence imaging and PTT both in vitro and in vivo (Fig. 19a and b). ${ }^{371}$

In another study, Thakut and co-workers developed highly crystalline GQDs with good physicochemical and NIR optical properties via the carbonization of Ficus racemosa leaves, which is a cluster fig tree native to India. ${ }^{372}$ The production yield of GQDs was $18 \%$ with a QY of about $14.16 \%$. The synthesized GQDs exhibited good compatibility and photostability in both organic and aqueous media. The potential of these GQDs to act as in vivo fluorescent probes was confirmed via a cytotoxicity analysis. The generation of ROS in a concentration-dependent manner combined with PTT activity was observed after excitation with $808 \mathrm{~nm}$ laser. The GQDs were stable for $30 \mathrm{~min}$ under continuous laser irradiation. These highly fluorescent GQDs were also used for PTT of MDA-MB-231 breast cancer cells.

Tian and co-workers synthesized a composite derived from metal-organic frameworks combined with GQDs. They used zeolitic imidazolate framework-8 (ZIF-8) as a drug nanocarrier plus embedded GQDs as the PTT chromophore. The drug release performance, PTT efficacy and synergistic therapeutic efficacy were systematically investigated using Dox as a model anticancer drug. The Dox-loaded ZIF-8/GQD nanoparticles could transform NIR irradiation into heat. Using $4 \mathrm{~T} 1$ breast cancer cells as a model, they showed the synergistic effect of the combined chemotherapy and PTT compared with either chemotherapy or PTT used alone (Fig. 20a). ${ }^{373}$

Liu and co-workers reported the fabrication of GQDs with strong absorption at $1070 \mathrm{~nm}$ in the NIR-II region. They used a one-step solvothermal process with phenol as the only

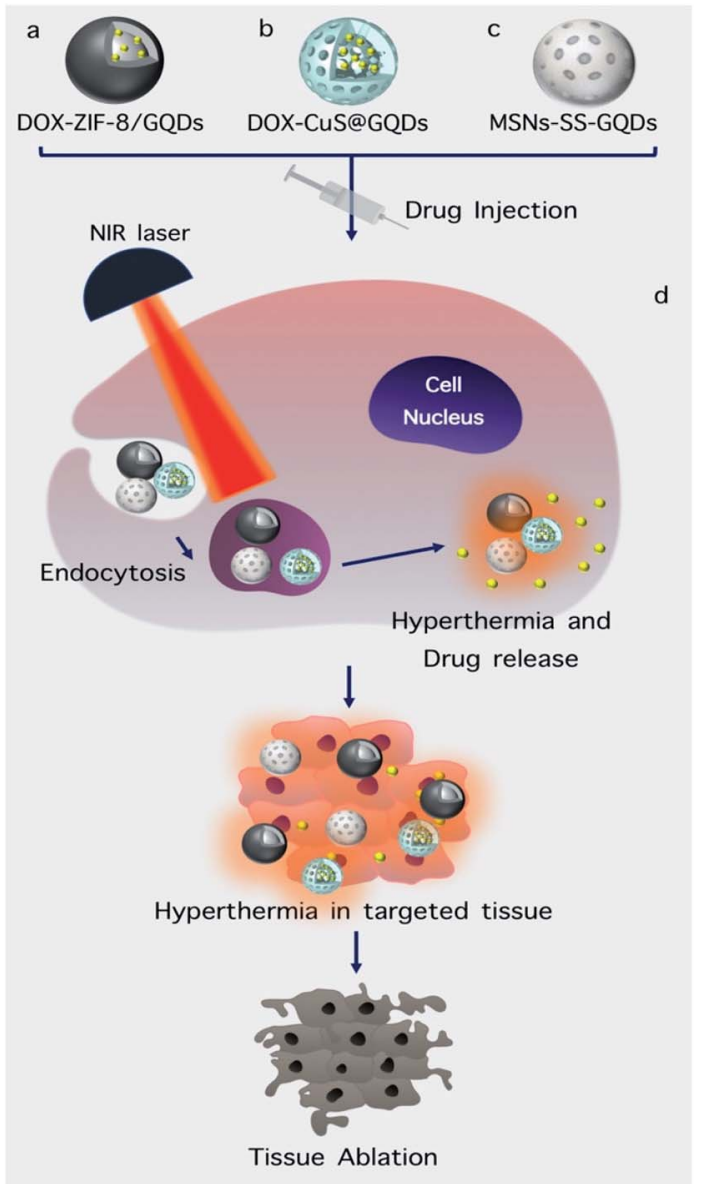

Fig. 20 (a and $d)$ Schematic illustration of the ZIF-8/GQD nanoparticles with encapsulation of Dox molecules and synergistic Dox delivery and photo thermal therapy. ${ }^{373}$ (b and d) Dox-CuSaGQDs nanoparticles and their therapeutic function. ${ }^{375}$ (c and d) Schematic illustration of drug loading and release from mesoporous silica nanoparticles capped with GQDs and their function. ${ }^{376}$ 
precursor. Their synthesis was controlled by the decomposition of hydrogen peroxide under a high magnetic field (9 T). Uniform size (3.6 nm), tunable quantum yield (16.67\%), and high PTT conversion efficiency $(33.45 \%)$ were the most important advantages reported by Liu and co-workers. ${ }^{374}$

Zheng et al. investigated a multifunctional platform composed of GQDs modified with hollow copper sulfide nanoparticles (CuSNPs) for controlled intracellular drug release and enhanced PTT-chemotherapy. Initially, the CuSNPs were synthesized and used as an encapsulating agent for Dox. Subsequently, GQDs were decorated on the surface of the CuSNPs. The beneficial properties of the nanoplatform include its good crystal structure, constant nanoscale size, colloidal stability, high drug encapsulation ability, and improved optical absorbance. Drug release could be triggered by high temperature and/or NIR laser irradiation. Confocal images and flow cytometry showed a significant level of NIR-triggered Dox release inside MDA-MB-231 cells. The combination of PTT and chemotherapy by Zheng et al. is illustrated in Fig. 20b. ${ }^{375}$

The synthesis of mesoporous silica nanoparticles (MSNs) capped with GQDs was reported by Gao and colleagues as a novel PTT and redox-responsive drug delivery system. An amidation reaction between cysteine and amino-functionalized MSNs was used to create disulfide bonds and Rhodamine B (RhB), a red fluorescent dye, was then incorporated into the mesoporous structure of MSNs as a model drug. Capping MSNs with GQDs acted as a blocking agent on the pores of mesoporous structure in order to control the release of the $\mathrm{RhB}$ cargo. The prepared nanocomposite demonstrated redox responsiveness to the presence of glutathione (GSH), which cleaved the disulfide bonds and released the loaded drug under redox control (Fig. 20c). ${ }^{376}$

7.6.3. Combined therapies. As mentioned before, biological systems are complex, and thus interfering with them in order to overcome a disease requires multifunctional approaches. Moreover, some diseases do not respond to monotherapies properly. In this case, combined therapies are a possible solution. GQDs and CQDs are suitable structures that can possess independent therapeutic functions besides drug delivery applications. Moreover, they can become stimuli responsive with some modifications. These properties make them promising candidates for combined therapy. Synergism of chemotherapy and photodynamic therapy is an example of this approach.

In this section, we summarize the recent reports of O-dotbased nanocomposites that have combined imaging with one or more other modalities, including phototherapy, chemotherapy, and computed tomography (CT) (Fig. 21).

Lan et al. synthesized CQDs with an absorption of up to $1100 \mathrm{~nm}$ via the hydrothermal treatment of 1,3,6-trinitropyrene and $\mathrm{Na}_{2} \mathrm{SO}_{3} \cdot{ }^{377}$ The CQDs could simultaneously provide strong fluorescence and generate ${ }^{1} \mathrm{O}_{2}$ through a two-photon excitation mechanism. An outstanding PTT conversion ability under $800 \mathrm{~nm}$ femtosecond pulsed laser irradiation was also observed. The wide absorption peak of the CQDs enabled them to be used as a photoacoustic (PA) imaging probe. In vitro and in vivo experiments showed that the fluorescent CQDs had good biocompatibility and they could be used as a versatile phototheranostic probe for PA/fluorescence imaging, and as a PDT/ PTT dual photoactive material with synergistic effects for killing cancer cells using a single NIR laser. ${ }^{377}$

Zhang and co-workers synthesized magneto-fluorescent $\mathrm{Fe}_{3} \mathrm{O}_{4} / \mathrm{CQDS}$ coated with single-walled carbon nanotubes (SWNTs) as a multifunctional theranostic material for targeted imaging plus chemo/photodynamic/photothermal triple-mode therapy. In this study, Dox as a model chemotherapy drug, was encapsulated in the porous structure of the SWCNTs-PEG$\mathrm{Fe}_{3} \mathrm{O}_{4} @ \mathrm{CQD}$ nanocarriers with high efficiency. These magnetofluorescent structures were then conjugated to the sgc8c DNA aptamer, which specifically recognizes the receptor tyrosineprotein kinase-like 7 (also known as colon carcinoma kinase 4, CCK4) for targeted dual mode fluorescence/magnetic resonance (MR) imaging. The multifunctional porous structure demonstrated the synergistic killing of lung cancer cells via PDT, PTT and rapid release of Dox under simultaneous NIR laser irradiation and low $\mathrm{pH}$.

In another study, Wo and co-workers synthesized GQDcoated hollow magnetic nanospheres (HMNSs) as a versatile system with a synergistic combination of magneto-mechanical, photothermal, photodynamic and chemotherapy of cancer. These mixed modalities structurally and physically destroyed cancer cells, where their morphology was visibly different from the cells killed by other therapies. HMNSs were coated with silica shells and linked to carboxylated GQDs in a core-shell structure. The synthesized composite was then loaded with Dox and stabilized with liposomes. The system was able to destroy cancer cells using four different therapeutic modalities in a synergistic manner including magnetic field-mediated mechanical stimulation, PTT damage, PDT toxicity, and chemotherapy. ${ }^{378}$

Xuan and colleagues functionalized the surface of GQDs with gold nanosphere clusters. They performed this process via a simple procedure, in which the reduction and stabilization of gold were both carried out by the GQDs themselves. They showed good dispersion, stability, excellent performance for photoacoustic imaging (PAI) and computed tomography (CT) imaging, low cytotoxicity, and PTT conversion efficiency of up to $51.31 \%$. Cellular and animal experiments showed that the targeted PAI/CT imaging of tumors could be enhanced by modification with folic acid (FA). The combination chemo-PTT therapy could be carried out through controlled Dox release from GQD under the influence of heat and the acidic environment of the tumor. Furthermore, the observed therapeutic effect was superior compared to all the single modes alone.

Body weight monitoring, hematoxylin and eosin (H\&E) staining of organs, and blood biochemical indicators demonstrated the good safety and low toxicity of the probe after injection. ${ }^{379}$

Badrigilan et al. synthesized nanoparticles from super paramagnetic iron oxide (SPIO) and bismuth oxide $\left(\mathrm{Bi}_{2} \mathrm{O}_{3}\right)$ and they coated them with GQDs for use as in vitro CT/MR dualmodal biomedical imaging probes and for cancer-specific PTT. These nanocomposites showed strong light absorbance with a wide-band in the NIR region. The photo-thermal 


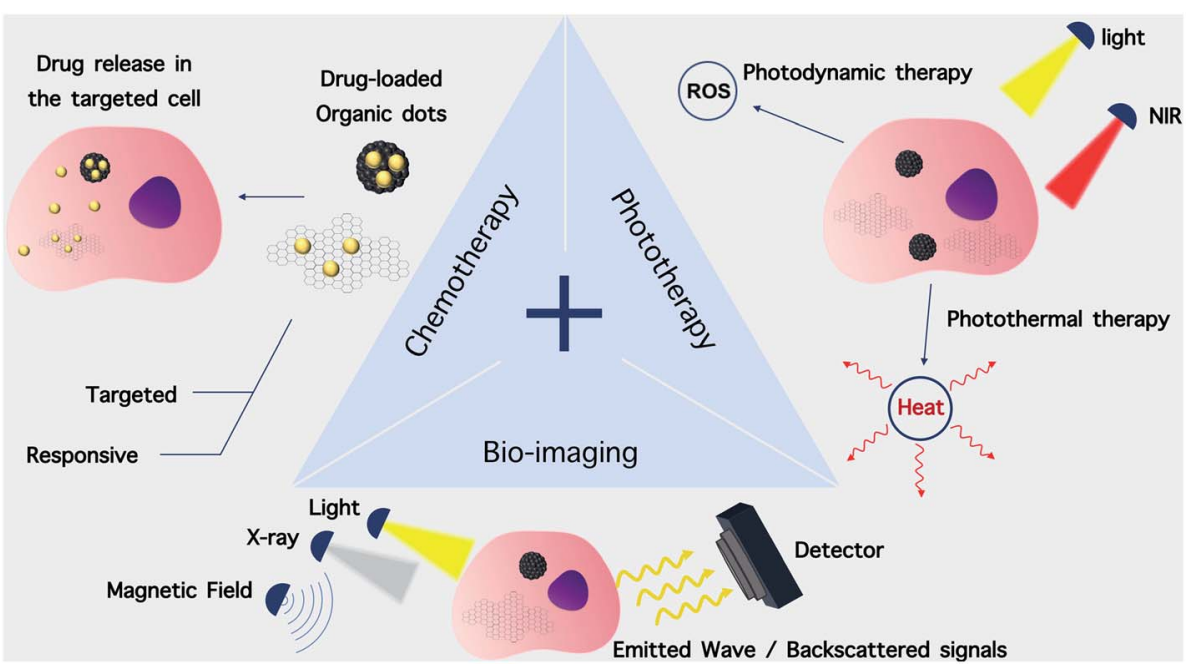

Fig. 21 Schematic illustration of combined therapy. Combined therapeutic systems possess 2 or 3 of these properties.

conversion efficacy $(\eta)$ was $31.8 \%$, with high photostability upon irradiation with $808 \mathrm{~nm}$ laser. The results of in vitro PTT of cancer cells showed a dose-dependent loss of viability of $\sim 53.4 \%$ in comparison to the laser alone group $(3.0 \%)$. The presence of Bi with a high atomic number $(Z=83)$ had good Xray attenuation capability $(175 \%)$ and strong shortening of the $T_{2}$-relaxation time. ${ }^{380}$

\section{Conclusions and future perspectives}

In this review, we summarized the recent progress in the design, properties, and theranostic applications of O-dots including graphene quantum dots and carbon quantum dots and introduce this new concept in order to facilitate new possibilities in the synthesis, preparation and application of these materials. These small-sized quantum dots have attracted much attention in theranostics due to their exceptional optical and chemical properties such as non-blinking, lowtoxicity and photostability. They are now being investigated in medical diagnostics and imaging, bio-sensing, drug/gene delivery, photoactivation, and light-activated therapy. Here, we highlight some future directions that can produce further advances in this field.

One of the reasons for the interest in O-dots is that their synthetic process is relatively simple, and their integration with other nanomaterials is easy. Despite the wide variety of approaches and precursors that have been employed for their fabrication, none of these nanostructures have been transitioned into a marketable product to date. Better controlled synthetic approaches, more precise characterization, and more extensive nanotoxicology studies will be required for effective translation. Because the fluorescence properties and the quantum yield are related to the overall composition and the presence of chemical groups on the surface of O-dots, standardization of the preparation procedures is urgently required.
Surface engineering is used to further functionalize O-dots by tailoring their surface coating with reactive groups for subsequent chemical modification. The reactive groups on the surface of O-dots can be reacted with the terminal groups of organic, polymeric, inorganic and biological materials via covalent bonds, electrostatic interactions and hydrogen bonds. Unfortunately, precisely controlled surface passivation has been challenging to date. The number of reactive groups capping the surface and the ratio of reactive groups on modifying molecules are difficult to be exactly controlled. In the future, scientists should continue to develop rational strategies for the preparation of O-dots with bright fluorescence emission in the red/ near-IR spectral regions to allow deeper optical imaging in tissue and decrease the interference from background autofluorescence. Therefore, efficient one-pot procedures for producing surface-passivated O-dots with high quantum yields need further attention.

O-dots can act as carriers for many pharmaceutical applications. Once functionalized with various polymers, they can serve as versatile biocompatible gene delivery vehicles. Moreover, they can be designed to have extended therapeutic lifetimes within the body. Despite some safety concerns about O-dots, many studies have reported their use in hybrid forms for biological applications, particularly in gene delivery. Hybridization approaches using biocompatible polymers have been tested in order to lower their potential toxicity and to increase their biocompatibility and applicability as multi-functional imaging probes and delivery vehicles. However, more systematic toxicology studies are needed to confirm the safety and understand the pharmacokinetics of O-dots.

Multifunctional combinational therapeutic applications, including PPT, PDT, photoacoustic therapy, MRI, chemotherapy, and computed tomography can increase therapeutic efficacy, overcome tumor resistance, and eradicate undesirable side effects. However, the practical applications of O-dots that rely on their optical properties, size and shape, surface chemistry will need more work before there is any hope of clinical 
applications. The ability to combine a number of imaging and therapeutic modalities within a single platform may still allow theranostics to become a reality in the coming years.

\section{Conflicts of interest}

There are no conflicts to declare.

\section{References}

1 A. L. Rivas, A. L. Hoogesteijn, A. Antoniades, M. Tomazou, T. Buranda, D. J. Perkins, J. M. Fair, R. Durvasula, F. O. Fasina, G. P. Tegos and M. H. V. Van Regenmortel, Front. Immunol., 2019, DOI: 10.3389/fimmu.2019.01258.

2 J. Visvader and G. J. Lindeman, Cell Stem Cell, 2012, DOI: 10.1016/j.stem.2012.05.007.

3 J. Zugazagoitia, C. Guedes, S. Ponce, I. Ferrer, S. MolinaPinelo and L. Paz-Ares, Clin. Ther., 2016, 38, 1551-1566.

4 W. H. Organization, Guide to Cancer Early Diagnosis, 2017.

5 P. Bhatkoti and M. Paul, IEEE Xplore, 2016, DOI: 10.1109/ IVCNZ.2016.7804459.

6 S. Kaur, M. J. Baine, M. Jain, A. R. Sasson and S. K. Batra, Biomarkers Med., 2012, 6, 597-612.

7 T. Goh, L. G. Goh, C. H. Ang and C. H. Wong, Br. J. Surg., 2014, 101, e119-e125.

8 G. Chen, H. Qiu, P. N. Prasad and X. Chen, Chem. Rev., 2014, 114, 5161-5214.

9 T. Peng, Z. Deng, J. He, Y. Li, Y. Tan, Y. Peng, X. Wang and W. Tan, Coord. Chem. Rev., 2020, 403, DOI: 10.1016/ j.ccr.2019.213080.

10 N. Gupta, D. Rai, A. Jangid and H. Kulhari, Methods Microbiol., 2019, 46, 143-172.

11 X. Le Hu, N. Kwon, K. C. Yan, A. C. Sedgwick, G. R. Chen, X. P. He, T. D. James and J. Yoon, Adv. Funct. Mater., 2020, 30, DOI: 10.1002/adfm.201907906.

12 Y. Zhang, K. Huang, J. Lin and P. Huang, Biomater. Sci., 2019, DOI: 10.1039/C8BM00642C.

13 B. Katzung, S. B. Masters, A. J. Trevor, Basic and clinical pharmacology, Mc Grow-Hill Medical, 12th edn, 2012.

14 E. Butcher, E. Berg and E. J. Kunkel, Nat. Biotechnol., 2004, 22, 1253-1259.

15 R. Parikh, A. Mathai, S. Parikh, G. C. Sekhar and R. Thomas, Indian J. Ophthalmol., 2008, 56, 45-50.

16 M. Rovaris, C. Confavreux, R. Furlan, L. Kappos, G. Comi and M. Filippi, Lancet Neurol., 2006, 5, 343-354.

17 X. Chen, S. S. Gambhir and J. Cheon, Acc. Chem. Res., 2011, 44, 841.

18 J. V. Jokerst and S. S. Gambhir, Acc. Chem. Res., 2011, 44, 1050-1060.

19 M. F. Kircher, U. Mahmood, R. S. King, R. Weissleder and L. Josephson, Cancer Res., 2003, 63, 8122-8125.

20 D. McDonald and P. L. Choyke, Nat. Med., 2003, 9, 713-725.

21 J.-C. G. Bünzli, Chem. Rev., 2010, 110, 2729-2755.

22 J.-C. G. Buenzli and S. V. Eliseeva, Chem. Sci., 2013, 4, 19391949.

23 A. Malek, O. Merkel, L. Fink, F. Czubayko, T. Kissel and A. Aigner, Toxicol. Appl. Pharmacol., 2009, 236, 97-108.
24 M. E. Davis, J. E. Zuckerman, C. H. J. Choi, D. Seligson, A. Tolcher, C. A. Alabi, Y. Yen, J. D. Heidel and A. Ribas, Nature, 2010, 464, 1067.

25 G. B. Jacobson, E. Gonzalez-Gonzalez, R. Spitler, R. Shinde, D. Leake, R. L. Kaspar, C. H. Contag and R. N. Zare, J. Pharm. Sci., 2010, 99, 4261-4266.

26 D. E. Dolmans, D. Fukumura and R. K. Jain, Nat. Rev. Cancer, 2003, 3, 380.

27 P. K. Jain, X. Huang, I. H. El-Sayed and M. A. El-Sayed, Acc. Chem. Res., 2008, 41, 1578-1586.

28 J.-H. Lee, Y.-M. Huh, Y. Jun, J. Seo, J. Jang, H.-T. Song, S. Kim, E.-J. Cho, H.-G. Yoon and J.-S. Suh, Nat. Med., 2007, 13, 95.

29 J. Xie, S. Lee and X. Chen, Adv. Drug Delivery Rev., 2010, 62, 1064-1079.

30 X. Gao, Y. Cui, R. M. Levenson, L. W. K. Chung and S. Nie, Nat. Biotechnol., 2004, 22, 969.

31 I. L. Medintz, H. T. Uyeda, E. R. Goldman and H. Mattoussi, Nat. Mater., 2005, 4, 435.

32 K. Welsher, Z. Liu, S. P. Sherlock, J. T. Robinson, Z. Chen, D. Daranciang and H. Dai, Nat. Nanotechnol., 2009, 4, 773.

33 P. N. Prasad, Introduction to biophotonics, John Wiley \& Sons, 2004.

34 P. N. Prasad, Nanophotonics, John Wiley \& Sons, 2004.

$35 \mathrm{P}$. N. Prasad, Introduction to nanomedicine and nanobioengineering, John Wiley \& Sons, 2012, vol. 7.

36 A. Schroeder, D. A. Heller, M. M. Winslow, J. E. Dahlman, G. W. Pratt, R. Langer, T. Jacks and D. G. Anderson, Nat. Rev. Cancer, 2012, 12, 39.

37 A. S. Thakor and S. S. Gambhir, Ca-Cancer J. Clin., 2013, 63, 395-418.

38 C. H. Lee, R. Rajendran, M.-S. Jeong, H. Y. Ko, J. Y. Joo, S. Cho, Y. W. Chang and S. Kim, Chem. Commun., 2013, 49, 6543-6545.

39 A. De La Zerda, C. Zavaleta, S. Keren, S. Vaithilingam, S. Bodapati, Z. Liu, J. Levi, B. R. Smith, T.-J. Ma and O. Oralkan, Nat. Nanotechnol., 2008, 3, 557.

40 J.-W. Kim, E. I. Galanzha, E. V. Shashkov, H.-M. Moon and V. P. Zharov, Nat. Nanotechnol., 2009, 4, 688.

41 Z. Liu, X. Li, S. M. Tabakman, K. Jiang, S. Fan and H. Dai, J. Am. Chem. Soc., 2008, 130, 13540-13541.

42 Z. Liu, C. Davis, W. Cai, L. He, X. Chen and H. Dai, Proc. Natl. Acad. Sci. U. S. A., 2008, 105, 1410-1415.

43 S. Y. Lim, W. Shen and Z. Gao, Chem. Soc. Rev., 2015, 44, 362-381.

44 P. Miao, K. Han, Y. Tang, B. Wang, T. Lin and W. Cheng, Nanoscale, 2015, 7, 1586-1595.

45 J. Zhang and S. H. Yu, Mater. Today, 2016, 19, 382-393.

46 S. Zhu, Q. Meng, L. Wang, J. Zhang, Y. Song, H. Jin, K. Zhang, H. Sun, H. Wang and B. Yang, Angew. Chem., 2013, 125, 4045-4049.

47 L. Cao, X. Wang, M. J. Meziani, F. Lu, H. Wang, P. G. Luo, Y. Lin, B. A. Harruff, L. M. Veca and D. Murray, J. Am. Chem. Soc., 2007, 129, 11318-11319.

48 C. Ding, A. Zhu and Y. Tian, Acc. Chem. Res., 2013, 47, 2030. 
49 B. Kong, A. Zhu, C. Ding, X. Zhao, B. Li and Y. Tian, Adv. Mater., 2012, 24, 5844-5848.

50 X. Sun, Z. Liu, K. Welsher, J. T. Robinson, A. Goodwin, S. Zaric and H. Dai, Nano Res., 2008, 1, 203-212.

51 J. Tang, B. Kong, H. Wu, M. Xu, Y. Wang, Y. Wang, D. Zhao and G. Zheng, Adv. Mater., 2013, 25, 6569-6574.

52 M. Bacon, S. J. Bradley and T. Nann, Part. Part. Syst. Charact., 2014, 31, 415-428.

53 H. Sun, L. Wu, W. Wei and X. Qu, Mater. Today, 2013, 16, 433-442.

54 Z. Fan, S. Li, F. Yuan and L. Fan, RSC Adv., 2015, 5, 1977319789.

55 P. Tian, L. Tang, K. Teng and S. Lau, Mater. Today Chem., 2018, 10, 221-258.

56 M. Li, T. Chen, J. J. Gooding and J. Liu, ACS Sens., 2019, 4, 1732-1748.

57 S. Campuzano, P. Yáñez-Sedeño and J. M. Pingarrón, Nanomaterials, 2019, DOI: 10.3390/nano9040634.

58 M. J. Molaei, RSC Adv., 2019, 9, 6460-6481.

59 K. Nekoueian, M. Amiri, M. Sillanpää, F. Marken, R. Boukherroub and S. Szunerits, Chem. Soc. Rev., 2019, 48, 4281-4316.

60 X. Zhao, W. Gao, H. Zhang, X. Qiu and Y. Luo, Modern Trends in Analysis, 2020, 493-505.

61 R. Wang, K. Lu, Z. Tang and Y Xu, J. Mater. Chem. A, 2017, 5, 3717-3734.

62 N. M. Zholobak, A. L. Popov, A. B. Shcherbakov, N. R. Popova, M. M. Guzyk, V. P. Antonovich, A. V. Yegorova, Y. V. Scrypynets, I. I. Leonenko and A. Y. Baranchikov, Beilstein J. Nanotechnol., 2016, 7, 19051917.

63 M. O. Dekaliuk, O. Viagin, Y. V. Malyukin and A. P. Demchenko, Phys. Chem. Chem. Phys., 2014, 16, 16075-16084.

64 R. Yan, H. Wu, Q. Zheng, J. Wang, J. Huang, K. Ding, Q. Guo and J. Wang, RSC Adv. , 2013, 4, 23097-23106.

65 R. Yan, H. Wu, Q. Zheng, J. Wang, J. Huang, K. Ding, Q. Guo and J. Wang, RSC Adv. , 2014, 4, 23097-23106.

66 A. Barras, Q. Pagneux, F. Sane, Q. Wang, R. Boukherroub, D. Hober and S. Szunerits, ACS Appl. Mater. Interfaces, 2016, 8, 9004-9013.

67 N. Wang, Y. Wang, T. Guo, T. Yang, M. Chen and J. Wang, Biosens. Bioelectron., 2016, 85, 68-75.

68 K. Nekoueian, M. Amiri, M. Sillanpää, F. Marken, R. Boukherroub and S. Szunerits, Chem. Soc. Rev., 2019, 48, 4281-4316.

69 Z. L. Wu, M. X. Gao, T. T. Wang, X. Y. Wan, L. L. Zheng and C. Z. Huang, Nanoscale, 2014, 6, 3868-3874.

70 X. Tan, Y. Li, X. Li, S. Zhou, L. Fan and S. Yang, Chem. Commun., 2015, 51, 2544-2546.

71 S. Qu, X. Wang, Q. Lu, X. Liu and L. Wang, Angew. Chem., 2012, 124, 12381-12384.

72 S. Zhu, S. Tang, J. Zhang and B. Yang, Chem. Commun., 2012, 48, 4527-4539.

73 Z. Fan, Y. Li, X. Li, L. Fan, S. Zhou, D. Fang and S. Yang, Carbon, 2014, 70, 149-156.
74 D. Pan, L. Guo, J. Zhang, C. Xi, Q. Xue, H. Huang, J. Li, Z. Zhang, W. Yu and Z. Chen, J. Mater. Chem., 2012, 22, 3314-3318.

75 L. Tang, R. Ji, X. Cao, J. Lin, H. Jiang, X. Li, K. S. Teng, C. M. Luk, S. Zeng and J. Hao, ACS Nano, 2012, 6, 51025110.

76 H. Qin, T. Gong, Y. Jin, Y. Cho, C. Shin, C. Lee and T. Kim, Carbon, 2015, 94, 181-188.

77 H. Tetsuka, R. Asahi, A. Nagoya, K. Okamoto, I. Tajima, R. Ohta and A. Okamoto, Adv. Mater., 2012, 24, 5333-5338.

78 M. D. Nurunnabi, Z. Khatun, G. R. Reeck, D. Y. Lee and Y. Lee, Chem. Commun., 2013, 49, 5079-5081.

79 F. Yuan, L. Ding, Y. Li, X. Li, L. Fan, S. Zhou, D. Fang and S. Yang, Nanoscale, 2015, 7, 11727-11733.

80 Y. Li, Y. Zhao, H. Cheng, Y. Hu, G. Shi, L. Dai and L. Qu, J. Am. Chem. Soc., 2011, 134, 15-18.

81 Y. Dong, H. Pang, S. Ren, C. Chen, Y. Chi and T. Yu, Carbon, 2013, 64, 245-251.

82 J. Ge, M. Lan, B. Zhou, W. Liu, L. Guo, H. Wang, Q. Jia, G. Niu, X. Huang and H. Zhou, Nat. Commun., 2014, 5, 4596.

83 X. Li, H. Wang, Y. Shimizu, A. Pyatenko, K. Kawaguchi and N. Koshizaki, Chem. Commun., 2010, 47, 932-934.

84 Y. H. Yuan, Z. X. Liu, R. S. Li, H. Y. Zou, M. Lin, H. Liu and C. Z. Huang, Nanoscale, 2016, 8, 6770-6776.

85 J. Zong, X. Yang, A. Trinchi, S. Hardin, I. Cole, Y. Zhu, C. Li, T. Muster and G. Wei, Nanoscale, 2013, 5, 11200-11206.

86 L. Deng, L. Liu, C. Zhu, D. Li and S. Dong, Chem. Commun., 2013, 49, 2503-2505.

87 Y.-P. Sun, B. Zhou, Y. Lin, W. Wang, K. A. S. Fernando, P. Pathak, M. J. Meziani, B. A. Harruff, X. Wang and H. Wang, J. Am. Chem. Soc., 2006, 128, 7756-7757.

88 Z. L. Wu, Z. X. Liu and Y. H. Yuan, J. Mater. Chem. B, 2017, 5, 3794-3809.

89 L. Shen, L. Zhang, M. Chen, X. Chen and J. Wang, Carbon, 2013, 55, 343-349.

90 X. Jia, J. Li and E. Wang, Nanoscale, 2012, 4, 5572-5575.

91 L. Zhou, Z. Li, Z. Liu, J. Ren and X. Qu, Langmuir, 2013, 29, 6396-6403.

92 M. A. Sk, A. Ananthanarayanan, L. Huang, K. H. Lim and P. Chen, J. Mater. Chem. C, 2014, 2, 6954-6960.

93 L. I. Cao, M. J. Meziani, S. Sahu and Y.-P. Sun, Acc. Chem. Res., 2012, 46, 171-180.

94 H. Li, Z. Kang, Y. Liu and S.-T. Lee, J. Mater. Chem., 2012, 22, 24230-24253.

95 Y. Zhang, Y. Zhang, G. Hong, W. He, K. Zhou, K. Yang, F. Li, G. Chen, Z. Liu and H. Dai, Biomaterials, 2013, 34, 36393646.

96 A. Nel, T. Xia, H. Meng, X. Wang, S. Lin, Z. Ji and H. Zhang, Acc. Chem. Res., 2012, 46, 607-621.

97 Y. Zhang, G. Hong, Y. Zhang, G. Chen, F. Li, H. Dai and Q. Wang, ACS Nano, 2012, 6, 3695-3702.

98 M. Havrdova, K. Hola, J. Skopalik, K. Tomankova, M. Petr, K. Cepe, K. Polakova, J. Tucek, A. B. Bourlinos and R. Zboril, Carbon, 2016, 99, 238-248.

99 J. Yan, S. Hou, Y. Yu, Y. Qiao, T. Xiao, Y. Mei, Z. Zhang, B. Wang, C. C. Huang, C. H. Lin and G. Suo, Colloids Surf., B, 2018, 1171, 241-249. 
100 Y. Kang, Y. Li, Y. Fang, Y. Xu, X. Wei and X. Yin, Sci. Rep., 2015, 5, DOI: 10.1038/srep11835.

101 S. Li, Z. Guo, R. Feng, Y. Zhang, W. Xue and Z. Liu, RSC Adv., 2017, 7, 4975-4982.

102 X. Wei, L. Li, J. Liu, L. Yu, H. Li, F. Cheng, X. Yi, J. He and B. Li, ACS Appl. Mater. Interfaces, 2019, 11, 9832-9840.

103 X. W. Hua, Y. W. Bao, Z. Chen and F. G. Wu, Nanoscale, 2017, 9, 10948-10960.

104 D. Wang, L. Zhu, J.-F. Chen and L. Dai, Nanoscale, 2015, 7, 9894.

105 Z. M. Markovic, B. Z. Ristic, K. M. Arsikin, D. G. Klisic, L. M. Harhaji-Trajkovic, B. M. Todorovic-Markovic, D. P. Kepic, T. K. Kravic-Stevovic, S. P. Jovanovic and M. M. Milenkovic, Biomaterials, 2012, 33, 7084-7092.

106 Y. Yang, C. Xuejing and Y. Xin, Artic. J. Environ. Sci., 2019, 77, 198-209.

107 D. Jiang, Y. Chen, N. Li, W. Li, Z. Wang, J. Zhu, H. Zhang, B. Liu and S. Xu, PLoS One, 2015, DOI: 10.1371/ journal.pone.0144906.

108 Z. Fan, Y. Li, X. Li, L. Fan, S. Zhou, D. Fang and S. Yang, Carbon, 2014, 70, 149-156.

109 Y. Chong, Y. Ma, H. Shen, X. Tu, X. Zhou, J. Xu, J. Dai, S. Fan and Z. Zhang, Biomaterials, 2014, 35, 5041-5048.

110 H. Liu, T. Ye and C. Mao, Angew. Chem., Int. Ed., 2007, 46, 6473-6475.

111 D. Reyes, M. Camacho, M. Camacho, M. Mayorga, D. Weathers, G. Salamo, Z. Wang and A. Neogi, Nanoscale Res. Lett., 2016, 11, DOI: 10.1186/s11671-016-1638-8.

112 C. Hu, Y. Liu, Y. Yang, J. Cui, Z. Huang, Y. Wang, L. Yang, H. Wang, Y. Xiao and J. Rong, J. Mater. Chem. B, 2013, 1(1), 39-42.

113 D. B. Shinde and V. K. Pillai, Chem.-Eur. J., 2012, 18, 1252212528.

114 C. Wang, W. Wu, A. Periasamy and H. Chang, Green Chem., 2014, 16, 2509-2514.

115 S. Dey, A. Govindaraj, K. Biswas and C. Rao, Chem. Phys. Lett., 2014, 596, 203-208.

116 L. Tang, R. Ji, X. Cao, J. Lin, H. Jiang, X. Li, K. S. Teng, C. M. Luk, S. Zeng, J. Hao and S. P. Lau, ACS Nano, 2012, 6, 5102-5110.

117 J. Wang, C. F. Wang and S. Chen, Angew. Chem., Int. Ed., 2012, 51, 9297-9301.

118 J. Zhou, C. Booker, R. Li, X. Zhou, T. K. Sham, X. Sun and Z. Ding, J. Am. Chem. Soc., 2007, 129, 744-745.

119 Q. L. Zhao, Z. L. Zhang, B. H. Huang, J. Peng, M. Zhang and D. W. Pang, Chem. Commun., 2008, 41, 5116-5118.

120 H. Li, X. He, Z. Kang, H. Huang, Y. Liu, J. Liu, S. Lian, C. H. A. Tsang, X. Yang and S. T. Lee, Angew. Chem., Int. Ed., 2010, 49, 4430-4434.

121 N. Arora and N. Sharma, Diamond Relat. Mater., 2014, 50, 135-150.

122 H. Liu, T. Ye and C. Mao, Angew. Chem., Int. Ed., 2007, 46, 6473-6475.

123 G. K. Yogesh, E. P. Shuaib and D. Sastikumar, Mater. Res. Bull., 2017, 91, 220-226.

124 N. Tarasenka, A. Stupak, N. Tarasenko, S. Chakrabarti and D. Mariotti, ChemPhysChem, 2017, 18, 1074-1083.
125 A. B. Bourlinos, A. Stassinopoulos, D. Anglos, R. Zboril, M. Karakassides and E. P. Giannelis, Small, 2008, 4, 455458.

126 P.-C. Hsu, Z.-Y. Shih, C.-H. Lee and H.-T. Chang, Green Chem., 2012, 14, 917-920.

127 C.-W. Lai, Y.-H. Hsiao, Y.-K. Peng and P.-T. Chou, J. Mater. Chem., 2012, 22, 14403-14409.

128 X. Wu, F. Tian, W. Wang, J. Chen, M. Wu and J. X. Zhao, J. Mater. Chem. C, 2013, 1, 4676-4684.

129 Y. Dong, J. Shao, C. Chen, H. Li, R. Wang, Y. Chi, X. Lin and G. Chen, Carbon, 2012, 50, 4738-4743.

$130 \mathrm{~J} . \mathrm{Ju}$ and W. Chen, Biosens. Bioelectron., 2014, 58, 219-225.

131 Y. Deng, D. Zhao, X. Chen, F. Wang, H. Song and D. Shen, Chem. Commun., 2013, 49, 5751-5753.

132 J. Wang, C. Wang and S. Chen, Angew. Chem., 2012, 124, 9431-9435.

133 J. Kim and J. S. Suh, ACS Nano, 2014, 8, 4190-4196.

134 J.-Y. Yin, H.-J. Liu, S. Jiang, Y. Chen and Y. Yao, ACS Macro Lett., 2013, 2, 1033-1037.

135 X. T. Zheng, A. Ananthanarayanan, K. Q. Luo and P. Chen, Small, 2015, 11, 1620-1636.

136 M. Larhed, C. Moberg and A. Hallberg, Acc. Chem. Res., 2002, 35, 717-727.

137 F. Li, C. Li, X. Liu, Y. Chen, T. Bai, L. Wang, Z. Shi and S. Feng, Chem.-Eur. J., 2012, 18, 11641-11646.

138 H. Zhu, X. Wang, Y. Li, Z. Wang, F. Yang and X. Yang, Chem. Commun., 2009, 5118-5120.

139 H. Liu, Z. He, L.-P. Jiang and J.-J. Zhu, ACS Appl. Mater. Interfaces, 2015, 7, 4913-4920.

140 Z. Qian, J. Ma, X. Shan, L. Shao, J. Zhou, J. Chen and H. Feng, RSC Adv., 2013, 3, 14571-14579.

141 X. T. Zheng, A. Ananthanarayanan, K. Q. Luo and P. Chen, Small, 2015, 11, 1620-1636.

142 J. Feng, H. Dong, L. Yu and L. Dong, J. Mater. Chem. C, 2017, 5, 5984-5993.

143 S. Zhu, J. Zhang, S. Tang, C. Qiao, L. Wang, H. Wang, X. Liu, B. Li, Y. Li and W. Yu, Adv. Funct. Mater., 2012, 22, 47324740.

144 S. Hu, R. Tian, L. Wu, Q. Zhao, J. Yang, J. Liu and S. Cao, Chem.-Asian J., 2013, 8, 1035-1041.

145 H. Sun, L. Wu, N. Gao, J. Ren and X. Qu, ACS Appl. Mater. Interfaces, 2013, 5, 1174-1179.

146 H. Jiang, F. Chen, M. G. Lagally and F. S. Denes, Langmuir, 2009, 26, 1991-1995.

147 C. Hu, Y. Liu, Y. Yang, J. Cui, Z. Huang, Y. Wang, L. Yang, H. Wang, Y. Xiao and J. Rong, J. Mater. Chem. B, 2013, 1(1), 39-42.

148 Z. Qian, X. Shan, L. Chai, J. Ma, J. Chen and H. Feng, ACS Appl. Mater. Interfaces, 2014, 6, 6797-6805.

149 K. S. Prasad, R. Pallela, D. Kim and Y. Shim, Part. Part. Syst. Charact., 2013, 30, 557-564.

150 D. Qu, M. Zheng, P. Du, Y. Zhou, L. Zhang, D. Li, H. Tan, Z. Zhao, Z. Xie and Z. Sun, Nanoscale, 2013, 5, 12272-12277.

151 Y. Dong, H. Pang, H. Bin Yang, C. Guo, J. Shao, Y. Chi, C. M. Li and T. Yu, Angew. Chem., Int. Ed., 2013, 52, 78007804 . 
152 M. Zheng, S. Liu, J. Li, D. Qu, H. Zhao, X. Guan, X. Hu, Z. Xie, X. Jing and Z. Sun, Adv. Mater., 2014, 26, 3554-3560. 153 X. Zhai, P. Zhang, C. Liu, T. Bai, W. Li, L. Dai and W. Liu, Chem. Commun., 2012, 48, 7955-7957.

154 F. Du, Y. Ming, F. Zeng, C. Yu and S. Wu, Nanotechnology, 2013, 24, 365101.

155 L. Cheng, Y. Li, X. Zhai, B. Xu, Z. Cao and W. Liu, ACS Appl. Mater. Interfaces, 2014, 6, 20487-20497.

156 D. Mazzier, M. Favaro, S. Agnoli, S. Silvestrini, G. Granozzi, M. Maggini and A. Moretto, Chem. Commun., 2014, 50, 6592-6595.

157 S. Chandra, S. Pradhan, S. Mitra, P. Patra, A. Bhattacharya, P. Pramanik and A. Goswami, Nanoscale, 2014, 6, 36473655.

158 Y. Wang, S. Kalytchuk, Y. Zhang, H. Shi, S. V. Kershaw and A. L. Rogach, J. Phys. Chem. Lett., 2014, 5, 1412-1420.

159 J. Bian, C. Huang, L. Wang, T. Hung, W. A. Daoud and R. Zhang, ACS Appl. Mater. Interfaces, 2014, 6, 4883-4890.

160 L. Zhu, X. Cui, J. Wu, Z. Wang, P. Wang, Y. Hou and M. Yang, Anal. Methods, 2014, 6, 4430-4436.

161 Y. Yang, J. Cui, M. Zheng, C. Hu, S. Tan, Y. Xiao, Q. Yang and Y. Liu, Chem. Commun., 2012, 48, 380-382.

162 Z. Ye, R. Tang, H. Wu, B. Wang, M. Tan and J. Yuan, New J. Chem., 2014, 38, 5721-5726.

163 N. Gogoi and D. Chowdhury, J. Mater. Chem. B, 2014, 2, 4089-4099.

164 F. Wang, Z. Xie, B. Zhang, Y. Liu, W. Yang and C. Liu, Nanoscale, 2014, 6, 3818-3823.

165 X. Fan, N. Hua, J. Xu, Z. Wang, H. Jia and C. Wang, RSC Adv., 2014, 4, 16524-16527.

166 W. Liu, C. Li, Y. Ren, X. Sun, W. Pan, Y. Li, J. Wang and W. Wang, J. Mater. Chem. B, 2016, 4, 5772-5788.

167 C. X. Guo, D. Zhao, Q. Zhao, P. Wang and X. Lu, Chem. Commun., 2014, 50, 7318-7321.

168 B. C. M. Martindale, G. A. M. Hutton, C. A. Caputo and E. Reisner, J. Am. Chem. Soc., 2015, 137, 6018-6025.

169 Q. Liu, S. Xu, C. Niu, M. Li, D. He, Z. Lu, L. Ma, N. Na, F. Huang, H. Jiang and J. Ouyang, Biosens. Bioelectron., 2015, 64, 119-125.

170 M. Wu, Y. Wang, W. Wu, C. Hu, X. Wang, J. Zheng, Z. Li, B. Jiang and J. Qiu, Carbon, 2014, 78, 480-489.

171 S. Zhang, Q. Wang, G. Tian and H. Ge, Mater. Lett., 2014, 115, 233-236.

172 C. Fowley, A. P. McHale, B. McCaughan, A. Fraix, S. Sortino and J. F. Callan, Chem. Commun., 2015, 51, 81-84.

173 S. Maiti, K. Das and P. K. Das, Chem. Commun., 2013, 49, 8851-8853.

174 Y. Hao, Z. Gan, J. Xu, X. Wu and P. K. Chu, Appl. Surf. Sci., 2014, 311, 490-497.

175 K. Das, S. Maiti and P. K. Das, Langmuir, 2014, 30, 24482459.

176 K. K. R. Datta, O. Kozak, V. Ranc, M. Havrdova, A. B. Bourlinos, K. Šafářová, K. Hola, K. Tomankova, G. Zoppellaro and M. Otyepka, Chem. Commun., 2014, 50, 10782-10785.

177 K. Junka, J. Guo, I. Filpponen, J. Laine and O. J. Rojas, Biomacromolecules, 2014, 15, 876-881.
178 A. B. Bourlinos, R. Zbořil, J. Petr, A. Bakandritsos, M. Krysmann and E. P. Giannelis, Chem. Mater., 2011, 24, 6-8.

179 X. Liu, N. Zhang, T. Bing and D. Shangguan, Anal. Chem., 2014, 86, 2289-2296.

180 W. Kong, H. Wu, Z. Ye, R. Li, T. Xu and B. Zhang, J. Lumin., 2014, 148, 238-242.

181 A. Quaranta, S. Carturan, A. Campagnaro, M. Dalla Palma, M. Giarola, N. Daldosso, G. Maggioni and G. Mariotto, Thin Solid Films, 2014, 553, 188-192.

182 A. Gupta and C. K. Nandi, Sens. Actuators, B, 2017, 245, 137145.

183 Z. Su, H. Shen, H. Wang, J. Wang, J. Li, G. U. Nienhaus, L. Shang and G. Wei, Adv. Funct. Mater., 2015, 25, 54725478.

184 F. Chiti and C. M. Dobson, Annu. Rev. Biochem., 2006, 75, DOI: 10.1146/annurev.biochem.75.101304.123901.

185 M. Li, S. E. Howson, K. Dong, N. Gao, J. Ren, P. Scott and X. Qu, J. Am. Chem. Soc., 2014, 136, 11655-11663.

186 C. Glabe, Neurobiol. Aging, 2006, 27, 570-575.

187 X. Han, J. Park, W. Wu, A. Malagon, L. Wang, E. Vargas, A. Wikramanayake, K. N. Houk and R. Leblanc, Chem. Sci., 2017, 8, 2003-2009.

188 Y. Song, P. N. Cheng, L. Zhu, E. G. Moore and J. S. Moore, J. Am. Chem. Soc., 2014, 136, 5233-5236.

189 S. Palmal, N. R. Jana and N. R. Jana, J. Phys. Chem. C, 2014, 118, 21630-21638.

190 J. A. Yang, B. J. Johnson, S. Wu, W. S. Woods, J. M. George and C. J. Murphy, Langmuir, 2013, 29, 4603-4615.

191 C. Cabaleiro-Lago, F. Quinlan-Pluck, I. Lynch, S. Lindman, A. M. Minogue, E. Thulin, D. M. Walsh, K. A. Dawson and S. Linse, J. Am. Chem. Soc., 2008, 130, 15437-15443.

192 C. Li and R. Mezzenga, Nanoscale, 2013, 5, 6207-6218.

193 S. Li, L. Wang, C. C. Chusuei, V. M. Suarez, P. L. Blackwelder, M. Micic, J. Orbulescu and R. M. Leblanc, Chem. Mater., 2015, 27, 1764-1771.

194 S. Li, Z. Peng and R. M. Leblanc, Anal. Chem., 2015, 87, 6455-6459.

195 C. Frieden, Protein Sci., 2007, 16, 2334-2344.

196 S. Zhu, T. Lu, G. Zhang, J. Xu, Y. Song, Y. Li, L. Wang, B. Yang and F. Li, J. Mater. Chem. B, 2016, 4, 4913-4921.

197 S. Radic, T. Davis, P. Ke and F. Ding, RSC Adv., 2015, 5, 105489-105498.

198 M. Yousaf, H. Huang, P. Li, C. Wang and Y. Yang, ACS Chem. Neurosci., 2017, 8, 1368-1377.

199 G. Rusciano, A. De Luca, G. Pesce and A. Sasso, Carbon, 2009, 47, 2950-2957.

200 S. Nandi, R. Malishev, K. P. Kootery, S. Kolusheva and R. Jelinek, Chem. Commun., 2014, 50, 10299-10302.

201 S. Nandi, R. Malishev, S. K. Bhunia, S. Kolusheva, J. Jopp and R. Jelinek, Biophys. J., 2016, 110, 2016-2025.

202 S. Nandi, M. Ritenberg and R. Jelinek, Analyst, 2015, 140, 4232-4237.

203 T. K. Mandal and N. Parvin, J. Biomed. Nanotechnol., 2011, 7, 846-848. 
204 S. K. Misra, I. Srivastava, I. Tripathi, E. Daza, F. Ostadhossein and D. Pan, J. Am. Chem. Soc., 2017, 139, 1746-1749.

205 M. J. Meziani, X. Dong, L. Zhu, L. P. Jones, G. E. LeCroy, F. Yang, S. Wang, P. Wang, Y. Zhao and L. Yang, ACS Appl. Mater. Interfaces, 2016, 8, 10761-10766.

206 W. Bing, H. Sun, Z. Yan, J. Ren and X. Qu, Small, 2016, 12, $4713-4718$.

207 N. Zhou, S. Zhu, S. Maharjan, Z. Hao, Y. Song, X. Zhao, Y. Jiang, B. Yang and L. Lu, RSC Adv., 2014, 4, 62086-62095.

208 T. Wang, S. Zhu and X. Jiang, Toxicol. Res., 2015, 4, 885-894. 209 A. H. Loo, Z. Sofer, D. Bouša, P. Ulbrich, A. Bonanni and M. Pumera, ACS Appl. Mater. Interfaces, 2016, 8, 1951-1957. 210 H. Sun, J. Ren and X. Qu, Acc. Chem. Res., 2016, 49, 461-470. 211 L. Feng, A. Zhao, J. Ren and X. Qu, Nucleic Acids Res., 2013, 41, 7987-7996.

212 V. Milosavljevic, H. V. Nguyen, P. Michalek, A. Moulick, P. Kopel, R. Kizek and V. Adam, Chem. Pap., 2015, 69, 192-201.

213 A. Rich and S. Zhang, Nat. Rev. Genet., 2003, 4, 566-572.

214 P. Pierrat, R. Wang, D. Kereselidze, M. Lux, P. Didier, A. Kichler, F. Pons and L. Lebeau, Biomaterials, 2015, 290-302.

215 Z. Peng, X. Han, S. Li, A. O. Al-Youbi, A. S. Bashammakh, M. S. El-Shahawi and R. M. Leblanc, Coord. Chem. Rev., 2017, 343, 256-277.

216 L. Wang, X. Wang, A. Bhirde, J. Cao, Y. Zeng, X. Huang, Y. Sun, G. Liu and X. Chen, Adv. Healthcare Mater., 2014, 3, 1203-1209.

217 X. Yang, Y. Wang, X. Shen, C. Su, J. Yang, M. Piao, F. Jia, G. Gao, L. Zhang and Q. Lin, J. Colloid Interface Sci., 2017, 492, 1-7.

218 L. Lu, L. Guo, X. Wang, T. Kang and S. Cheng, RSC Adv., 2016, 6, 33072-33075.

219 S. Lu, S. Guo, P. Xu, X. Li, Y. Zhao, W. Gu and M. Xue, Int. J. Nanomed., 2016, 11, 6325-6336.

220 E. Barbu, J. Tsibouklis and D. C. Górecki, Expert Opin. Drug Delivery, 2009, DOI: 10.1517/17425240902939143.

221 S. Bhaskar, F. Tian and T. Stoeger, Toxicology, 2010, 7, DOI: 10.1186/1743-8977-7-3.

222 H. Maeda, J. Wu, T. Sawa, Y. Matsumura and K. Hori, J. Control. Release, 2000, 65, 271-284.

223 K. K. Pulicherla and M. K. Verma, AAPS PharmSciTech, 2015, 16, 223-233.

224 A. R. Jones and E. V. Shusta, Pharm. Res., 2007, 24, 17591771.

225 S. Bhunia, A. Saha, A. Maity, S. Ray and N. Jana, Sci. Rep., 2013, 3, DOI: 10.1038/srep01473.

226 M. Zheng, S. Ruan, S. Liu, T. Sun, D. Qu, H. Zhao, Z. Xie, H. Gao, X. Jing and Z. Sun, ACS Nano, 2015, 9, 11455-11461.

227 S. Li, Z. Peng, A. M. Othman and R. M. Graham, Nanoscale, 2016, 8, DOI: 10.1039/C6NR05055G.

228 D. T. Wiley, P. Webster, A. Gale and M. E. Davis, Proc. Natl. Acad. Sci. U. S. A., 2013, DOI: 10.1073/pnas.1307152110.

229 S. Dixit, T. Novak, K. Miller, Y. Zhu, M. E. Kenney and A.-M. Broome, Nanoscale, 2014, 7, 1782-1790.
230 H. Xie, D. Ghoorah, Y. Shang, H. Shi, F. Liu, X. Yang, H. Xu and W. Jiang, PLoS One, 2012, 7(5), e37376.

231 S. Li, Z. Peng, J. Dallman, J. Baker, A. Othman. P. Blackwelder and R. Leblanc, Colloids Surf., $B, 2016,145,251-256$.

232 A. Kalueff, A. Stewart and R. Gerlai, Trends Pharmacol. Sci., 2014, 35, 63-75.

233 Z. Peng, E. Miyanji, Y. Zhou, J. Pardo, S. Hettiarachchi, S. Li, P. Blackwelder, I. Skromne and R. M. Leblanc, Nanoscale, 2017, 9, 17533-17543.

234 X. Han, Z. Jing, W. Wu, B. Zou, Z. Peng, P. Ren, A. Wikramanayake, Z. Lu and R. M. Leblanc, Nanoscale, 2017, 9, 12862-12866.

235 K. J. Mintz, G. Mercado, Y. Zhou, Y. Ji, S. D. Hettiarachchi, P. Y. Liyanage, R. R. Pandey, C. C. Chusuei, J. Dallman and R. M. Leblanc, Colloids Surf., B, 2019, 176, 488-493.

236 D. Kim, J. Yoo, H. Hwang, J. Lee and S. H. Lee, Nat. Nanotechnol., 2018, 13, 812-818.

237 K. L. Li, Y. H. Zhang, R. Xing, Y. F. Zhou, X. D. Chen, H. Wang, B. Song, Y. H. Sima, Y. He and S. Q. Xu, $R S C$ Adv., 2017, 7, 50317-50327.

238 C. Sarkar, A. R. Chowdhuri, A. Kumar, D. Laha, S. Garai, J. Chakraborty and S. K. Sahu, Carbohydr. Polym., 2018, 181, 710-718.

239 M. Zhang, Y. Zhao, J. Cheng, X. Liu, Y. Wang, X. Yan, Y. Zhang, F. Lu, Q. Wang and H. Qu, Artif. Cells, Nanomed., Biotechnol., 2017, 1-10.

240 X. Guo, L. Xu, L. Zhang, H. Wang, X. Wang, X. Liu, J. Yao and A. Hao, J. Lumin., 2018, 196, 100-110.

241 Y. Yuan, B. Guo, L. Hao, N. Liu, Y. Lin, W. Guo, X. Li and B. Gu, Colloids Surf., B, 2017, 159, 349-359.

242 Q. Wang, X. Huang, Y. Long, X. Wang, H. Zhang and R. Zhu, Carbon, 2013, 59, 192-199.

243 C.-L. Li, C.-M. Ou, C.-C. Huang, W.-C. Wu, Y.-P. Chen, T.-E. Lin, L.-C. Ho, C.-W. Wang, C.-C. Shih, H.-C. Zhou, Y.-C. Lee, W.-F. Tzeng, T.-J. Chiou and S.-T. Chu, J. Mater. Chem. B, 2014, 2, 4564-4571.

244 Y. Yuan, B. Guo, L. Hao, N. Liu, Y. Lin, W. Guo, X. Lee and B. Gu, Colloids Surf., B, 2017, 159, 349-359.

245 F. Khodadadei, S. Safarian and N. Ghanbari, Mater. Sci. Eng. C, 2017, 79, 280-285.

246 S. Some, A. Gwon, E. Hwang, G. Bahn, Y. Yoon, Y. Kim and S. Kim, Sci. Rep., 2014, 4, DOI: 10.1038/srep06314.

247 C. Rao, S. Khan, N. C. Verma and C. K. Nandi, ChemBioChem, 2017, 18, 2385-2389.

248 T. García-Mendiola, I. Bravo, J. M. López-Moreno, F. Pariente, R. Wannemacher, K. Weber, J. Popp and E. Lorenzo, Sens. Actuators, B, 2018, 256, 226-233.

249 W. Zhang, L. Shi, Y. Liu, X. Meng, H. Xu, Y. Xu, B. Liu, X. Fang, H.-B. Li and T. Ding, $R S C A d v ., 2017,7$, 2034520353.

250 N. Gao, W. Yang, H. Nie, Y. Gong, J. Jing, L. Gao and X. Zhang, Biosens. Bioelectron., 2017, 96, 300-307.

251 D. Lei, W. Yang, Y. Gong, J. Jing, H. Nie, B. Yu and X. Zhang, Sens. Actuators, B, 2016, 230, 714-720.

252 J. Zhang, X. Zhao, M. Xian, C. Dong and S. Shuang, Talanta, 2018, 183, 39-47. 
253 A. Zhao, Z. Chen, C. Zhao, N. Gao, J. Ren and X. Qu, Carbon, 2015, 85, 309-327.

254 M. X. Gao, L. Yang, Y. Zheng, X. X. Yang, H. Y. Zou, J. Han, Z. X. Liu, Y. F. Li and C. Z. Huang, Chem.-Eur. J., 2017, 23, 2171-2178.

255 H. Motaghi, M. A. Mehrgardi and P. Bouvet, Sci. Rep., 2017, 7, 10513.

256 D. Musumeci, C. Platella, C. Riccardi, F. Moccia and D. Montesarchio, Cancer, 2017, 9, 174.

257 L. Yang, Z. Wang, J. Wang, W. Jiang, X. Jiang, Z. Bai, Y. He, J. Jiang, D. Wang and L. Yang, Nanoscale, 2016, 8, 68016809.

258 J. Pardo, Z. Peng and R. M. Leblanc, Molecules, 2018, 23, 378.

259 T. Feng, X. Ai, H. Ong and Y. Zhao, ACS Appl. Mater. Interfaces, 2016, 8, 18732-18740.

260 X.-W. Hua, Y.-W. Bao, H.-Y. Wang, Z. Chen and F.-G. Wu, Nanoscale, 2017, 9, 2150-2161.

261 J. S. Sidhu, A. Singh, N. Garg, N. Kaur and N. Singh, Analyst, 2018, 143, 1853-1861.

262 S. Wang, C. Li, M. Qian, H. Jiang, W. Shi, J. Chen, U. Lächelt, E. Wagner, W. Lu and Y. Wang, Biomaterials, 2017, 141, 29-39.

263 E. Pugliese, J. Q. Coentro and D. I. Zeugolis, Adv. Mater., 2018, DOI: 10.1002/adma.201704324.

264 L. Yang, Z. Wang, J. Wang, W. Jiang, X. Jiang, Z. Bai and Y. He, Nanoscale, 2016, 8, 6801-6809.

265 X. Wang, X. Sun, J. Lao, H. He, T. Cheng, M. Wang, S. Wang and F. Huang, Colloids Surf., B, 2014, 122, 638-644.

266 R. Justin, S. Román, D. Chen, K. Tao, X. Geng and R. Grant, $R S C$ Adv., 2015, 5, DOI: 10.1039/C5RA04340A.

267 L. Ruiyi, L. Zaijun, S. Xiulan, J. Jan, L. Lin, G. Zhigou and W. Guangli, Chem. Eng. J., 2020, 382, DOI: 10.1016/ j.cej.2019.122992.

268 A. Chowdhury, A. Ganganboina, Y. Tsai, H. Chiu and R. Doong, Anal. Chim. Acta, 2018, 1027, 109-120.

269 R. Imani, F. Mohabatpour and F. Mostafavi, Carbon, 2018, 140, 569-591.

270 D. Scherman, A. Rousseau, P. Bigey and V. Escriou, Gene Ther., 2017, 24, DOI: 10.1038/gt.2017.6.

271 H. Yin, R. Kanasty, A. Eltoukhy, A. Vegas, R. Dorkin and D. Anderson, Nat. Rev. Genet., 2014, 15, 541-555.

272 M. K. Riley and W. Vermerris, Nanomaterials, 2017, DOI: 10.3390/nano7050094.

273 X. Loh, T. Lee, Q. Dou and G. Roshan Deen, Biomater. Sci., 2016, 4, 70-86.

274 J. Ageitos, J. Chuah and K. Numata, Nanomedicines, 2016, DOI: $10.1039 / 9781782622536-00001$.

275 A. Adler and K. Leong, Nano Today, 2010, 5, 553-569.

276 S. Jin and K. Ye, Biotechnol. Prog., 2007, 23, 32-41.

277 R. Misra, M. Upadhyay and S. Mohanty, Pharm. Anal. Acta, 2014, DOI: 10.4172/2153-2435.1000279.

278 V. Mundra and R. I. Mahato, Front. Chem. Sci. Eng., 2014, 8, 387-404.

279 A. Albanese, P. S. Tang and W. C. W. Chan, Annu. Rev. Biomed. Eng., 2012, 14, 1-16.
280 D. Yin, Y. Li, H. Lin, B. Guo, Y. Du, X. Li, H. Jia, X. Zhao, J. Tang and L. Zhang, Nanotechnology, 2013, DOI: 10.1088/ 0957-4484/24/10/105102.

281 A. Paul, A. Hasan, H. Al Kindi, A. K. Gaharwar, V. T. S. Rao, M. Nikkhah, S. R. Shin, D. Krafft, M. R. Dokmeci, D. ShumTim and A. Khademhosseini, ACS Nano, 2014, 8, 80508062.

282 R. Agarwal and K. Roy, Ther. Delivery, 2013, 4, 705-723.

283 J. Kim, L. J. Cote and J. Huang, Acc. Chem. Res., 2012, 45, 1356-1364.

284 V. C. Sanchez, A. Jachak, R. H. Hurt and A. B. Kane, Chem. Res. Toxicol., 2012, 25, 15-34.

285 C. Xu, D. Yang, L. Mei, B. Lu, L. Chen, Q. Li, H. Zhu and T. Wang, ACS Appl. Mater. Interfaces, 2013, 5, 2715-2724.

286 H. Tian, W. Xiong, J. Wei, Y. Wang, X. Chen, X. Jing and Q. Zhu, Biomaterials, 2007, 28, 2899-2907.

287 M. Ogris, P. Steinlein, M. Kursa, K. Mechtler, R. Kircheis and E. Wagner, The size of DNA/transferrin-PEI complexes is an important factor for gene expression in cultured cells, 1998, vol. 5 .

288 S. Ghafary, M. Nikkhah, S. Hatamie and S. Hosseinkhani, Sci. Rep., 2017, 7, DOI: 10.1038/s41598-017-09890-y.

289 H. Dong, W. Dai, H. Ju, H. Lu, S. Wang, L. Xu, S. F. Zhou, Y. Zhang and X. Zhang, ACS Appl. Mater. Interfaces, 2015, 7, 11015-11023.

290 Q. Wang, C. Zhang, G. Shen, H. Liu, H. Fu and D. Cui, J. Nanobiotechnol., 2014, 12, DOI: 10.1186/s12951-014-0058-0.

291 L. Zhang, W. Zheng, R. Tang, N. Wang, W. Zhang and X. Jiang, Biomaterials, 2016, 104, 269-278.

292 S. Kim, Y. Choi, G. Park, C. Won, Y. J. Park, Y. Lee, B. S. Kim and D. H. Min, Nano Res., 2017, 10, 503-519.

293 X. Yang, Y. Wang, X. Shen, C. Su, J. Yang and M. Piao, J. Colloid Interface Sci., 2017, 492, 1-7.

294 G. Mattheolabakis, L. Milane, A. Singh and M. M. Amiji, J. Drug Targeting, 2015, 23, 605-618.

295 H. J. Wang, J. Zhang, Y. H. Liu, T. Y. Luo, X. He and X. Q. Yu, RSC Adv., 2017, 7, 15613-15624.

296 P. G. Luo, S. Sahu, S. T. Yang, S. K. Sonkar, J. Wang, H. Wang, G. E. Lecroy, L. Cao and Y. P. Sun, J. Mater. Chem. B, 2013, 1, 2116-2127.

297 A. B. Bourlinos, A. Bakandritsos, A. Kouloumpis, D. Gournis, M. Krysmann, E. P. Giannelis, K. Polakova, K. Safarova, K. Hola and R. Zboril, J. Mater. Chem., 2012, 22, 23327-23330.

298 M. Nafiujjaman, H. Khan and Y. Lee, J. Nanosci. Nanotechnol., 2017, 17, DOI: 10.1166/jnn.2017.12825.

299 K. Li, X. Zhao, G. Wei and Z. Su, Curr. Med. Chem., 2017, 25, 2876-2893.

300 Y. Zhao, X. Hao, W. Lu, R. Wang, X. Shan, Q. Chen, G. Sun and J. Liu, ACS Appl. Nano Mater., 2018, 1, 2544-2551.

301 A. Dehghani, S. Ardekani, M. Hassan and V. Gomes, Carbon, 2018, 131, 238-245.

302 N. Vasimalai, V. Vilas-Boas and J. Gallo, Beilstein J. Nanotechnol., 2018, 9, 530-544.

303 G. Gao, Y. W. Jiang, H. R. Jia, J. Yang and F. G. Wu, Carbon, 2018, 134, 232-243. 
304 X. Chen, F. Gong, Z. Cao, W. Zou and T. Gu, Anal. Bioanal. Chem., 2018, 410, 2961-2970.

305 S. T. Yang, X. Wang, H. Wang, F. Lu, P. G. Luo, L. Cao, M. J. Meziani, J. H. Liu, Y. Liu, M. Chen, Y. Huang and Y. P. Sun, J. Phys. Chem. C, 2009, 113, 18110-18114.

306 S. V. Bozrova, M. A. Baryshnikova, Z. A. Sokolova, I. R. Nabiev and A. V. Sukhanova, KnE Energy, 2018, 58-63.

307 L. Cao, X. Wang, M. J. Meziani, F. Lu, H. Wang, P. G. Luo, Y. Lin, B. A. Harruff, L. M. Veca, D. Murray, S. Y. Xie and Y. P. Sun, J. Am. Chem. Soc., 2007, 129, 11318-11319.

308 R. Shereema, V. Sankar, K. Raghu and T. Rao, Electrochim. Acta, 2015, 182, 588-595.

309 Y. Zhang, M. Wu, Y. Wang, X. He, W. Li and X. Feng, Talanta, 2013, 117, 196-202.

310 S. C. Ray, A. Saha, N. R. Jana and R. Sarkar, J. Phys. Chem. C, 2009, 113, 18546-18551.

311 Y. Xu, M. Wu, Y. Liu, X.-Z. Feng, X.-B. Yin, X.-W. He and Y.-K. Zhang, Chem.-Eur. J., 2013, 19, 2276-2283.

312 G. Eda, Y.-Y. Lin, C. Mattevi, H. Yamaguchi, H.-A. Chen, I.-S. Chen, C.-W. Chen and M. Chhowalla, Adv. Mater., 2010, 22, 505-509.

313 L. Hu, Y. Sun, S. Li, X. Wang, K. Hu, L. Wang and X. Liang, Carbon, 2014, 67, 508-513.

314 W. Li, Z. Zhang, B. Kong, S. Feng, J. Wang, L. Wang, J. Yang, F. Zhang, P. Wu and D. Zhao, Angew. Chem., Int. Ed., 2013, 52, 8151-8155.

315 Z. A. Qiao, Y. Wang, Y. Gao, H. Li, T. Dai, Y. Liu and Q. Huo, Chem. Commun., 2010, 46, 8812-8814.

316 W. S. Zou, Y. J. Ji, X. F. Wang, Q. C. Zhao, J. Zhang, Q. Shao, J. Liu, F. Wang and Y. Q. Wang, Chem. Eng. J., 2016, 294, 323-332.

317 D. Kumar, K. Singh and V. Verma, J. Bionanosci., 2014, 8, 274-279.

318 V. Mehta, S. Jha, H. Basu, R. Singhal and S. Kailasa, Sens. Actuators, B, 2015, 213, 434-443.

319 Q. Liang, W. Ma, Y. Shi, Z. Li and X. Yang, Carbon, 2013, 60, 421-428.

320 L. Wang and H. S. Zhou, Anal. Chem., 2014, 86, 8902-8905.

321 C. Zhu, J. Zhai and S. Dong, Chem. Commun., 2012, 48, 9367-9369.

322 X. Yang, Y. Zhuo, S. Zhu, Y. Luo, Y. Feng and Y. Dou, Biosens. Bioelectron., 2014, 60, 292-298.

323 V. Mehta, S. Jha, H. Basu, R. Singhal and S. Kailasa, Sens. Actuators, B, 2014, 213, 434-443.

324 S. Sahu, B. Behera, T. Maiti and S. Mohapatra, Chem. Commun., 2012, 48, 8835-8837.

325 Z. Qian, J. Ma, X. Shan, H. Feng, L. Shao and J. Chen, Chem.-Eur. J., 2014, 20, 2254-2263.

326 G. C. Parker, M. Anastassova-Kristeva, L. M. Eisenberg, M. S. Rao, M. A. Williams, P. R. Sanberg and D. English, Stem Cells Dev., 2005, 14, 463-469.

327 A. Solanki, J. D. Kim and K. B. Lee, Nanomedicine, 2008, 3, 567-578.

328 M. Zhang, L. Bai, W. Shang, W. Xie, H. Ma, Y. Fu, D. Fang, H. Sun and L. Fan, J. Mater. Chem., 2012, 22, 7461-7467.

329 W. Shang, X. Zhang, M. Zhang, Z. Fan, Y. Sun, M. Han and L. Fan, Nanoscale, 2014, 6, 5799-5806.
330 Z. Fan, Y. Li, X. Li, L. Fan, S. Zhou, D. Fang and S. Yang, Carbon, 2014, 70, 149-156.

331 S. Zhu, J. Zhang, C. Qiao, S. Tang, Y. Li, W. Yuan, B. Li, L. Tian, F. Liu, R. Hu, H. Gao, H. Wei, H. Zhang, H. Sun and B. Yang, Chem. Commun., 2011, 47, 6858-6860.

332 J. Peng, W. Gao, B. K. Gupta, Z. Liu, R. Romero-Aburto, L. Ge, L. Song, L. B. Alemany, X. Zhan, G. Gao, S. A. Vithayathil, B. A. Kaipparettu, A. A. Marti, T. Hayashi, J. J. Zhu and P. M. Ajayan, Nano Lett., 2012, 12, 844-849.

333 Y. Dong, C. Chen, X. Zheng, L. Gao, Z. Cui, H. Yang, C. Guo, Y. Chi and C. M. Li, J. Mater. Chem., 2012, 22, 8764-8766.

334 X. Zhu, X. Xiao, X. Zuo, Y. Liang and J. Nan, Part. Part. Syst. Charact., 2014, 31, 801-809.

335 P. S. Saxena., A. Sirvastava, V. Kumar, V. Singh, S. Umrao, V. Parashar, S. Abraham, A. K. Singh and G. Nath, RSC $A d v .$, 2014, 4, 21101-21107.

336 X. Wang, X. Sun, J. Lao, H. He, T. Cheng, M. Wang, S. Wang and F. Huang, Colloids Surf., B, 2014, 122, 638-644.

337 J. Wang, Q. Li, J. Zhou, Y. Wang, L. Yu, H. Peng and J. Zhu, Materials, 2017, 72, 15-19.

338 C. Matea, T. Mocan, F. Tabaran, T. Pop and O. Mosteanu, Int. J. Nanomed., 2017, 12, 5421-5431.

339 G. Gu, C. Park, H. Cho, T. Ha, J. Bae and O. Kwon, Sci. Rep., 2018, 8, DOI: 10.1038/s41598-018-22828-2.

340 H. Tao, K. Yang, Z. Ma, J. Wan, Y. Zhang, Z. Kang and Z. Liu, Small, 2012, 8, 281-290.

341 L. Wu, M. Luderer, X. Yang, C. Swain and H. Zhang, Theranostics, 2013, 3, 677-686.

342 S. T. Yang, L. Cao, P. G. Luo, F. Lu, X. Wang, H. Wang, M. J. Meziani, Y. Liu, G. Qi and Y. P. Sun, J. Am. Chem. Soc., 2009, 131, 11308-11309.

343 K. Yang, S. Zhang, G. Zhang, X. Sun, S. T. Lee and Z. Liu, Nano Lett., 2010, 10, 3318-3323.

344 X. Wu, F. Tian, W. Wang, J. Chen, M. Wu and J. Zhao, J. Mater. Chem. C, 2013, 1, 4676-4684.

345 X. J. Ge, M. Lan, B. Zhou, W. Liu, L. Guo, H. Wang, Q. Jia, G. Niu, X. Huang, H. Zhou, X. Meng, P. Wang, C. S. Lee and X. Han, Nat. Commun., 2014, 5, 4596, DOI: 10.1038/ ncomms5596.

346 A. C. V. Doughty, A. R. Hoover, E. Layton, C. K. Murray, E. W. Howard and W. R. Chen, Materials, 2019, 12, DOI: 10.3390/ma12050779.

347 R. Singh and S. V. Torti, Adv. Drug Delivery Rev., 2013, 65, 2045-2060.

348 M. Kováčová, E. Špitalská, Z. Markovic and Z. Špitálský, Part. Part. Syst. Charact., 2020, 37, 1900348.

349 F. Wu, L. Yue, H. Su, K. Wang, L. Yang and X. Zhu, Nanoscale Res. Lett., 2018, 13, DOI: 10.1186/s11671-0182761-5.

350 T. J. Dougherty, C. J. Gomer, B. W. Henderson, G. Jori, D. Kessel, M. Korbelik, J. Moan and Q. Peng, J. Natl. Cancer Inst., 1998, 90, 889-905.

351 L. Yang, G. Xing, L. Wang, Y. Wu, S. Li, G. Xu, Q. He, J. Chen, M. Chen, X. Liu, Z. Zhu and L. Yang, Lancet, 2015, 1465-1471. 
352 M. Lyons, I. Phang, S. Eljamel and F. Ir, Photodiagn. Photodyn. Ther., 2012, 9, 40-45.

353 C. Kennedy and R. H. Pottier, J. Photochem. Photobiol., B, 1992, 14(4), 275-292.

354 A. P. Castano, T. N. Demidova and M. R. Hamblin, Photodiagn. Photodyn. Ther., 2004, DOI: 10.1016/S15721000(05)00007-4.

355 K. Yang, L. Hu, X. Ma, S. Ye, L. Cheng, X. Shi and C. Li, Adv. Mater., 2012, 24, 1868-1872.

356 S. Liao, X. Hu, Z. Liu, Y. Lin, R. Liang, Y. Zhang, Q. Li, Y. Li and X. Liao, Oncol. Lett., 2019, 17, 603-615.

357 T. A. Tabish, C. J. Scotton, D. C. J. Ferguson, L. Lin, A. Van Der, S. Lowry, M. Ali, F. Jabeen, M. Ali, G. Paul and S. Zhang, Nanomedicine, 2018, 13(15), 1923-1937.

358 M. Hassan, V. G. Gomes, A. Dehghani and S. M. Ardekani, Nano Res., 2018, 11, 1-41.

359 S. Brown, E. Brown and I. Walker, Lancet Oncol., 2004, 5, 497-508.

360 C. A. Leatherdale, W. K. Woo, F. V. Mikulec and M. G. Bawendi, J. Phys. Chem. B, 2002, 106, 7619-7622.

361 T. Nann, Engineering, 2011, 3, 137-143.

362 P. Yu, X. Wen, Y.-R. Toh and J. Tang, J. Phys. Chem. C, 2012, DOI: $10.1021 /$ jp307308z.

363 C. J. Reckmeier, J. Schneider, A. S. Susha and A. L. Rogach, Opt. Express, 2016, 24, A312.

364 P. Huang, J. Lin, X. Wang, Z. Wang, C. Zhang, M. He, K. Wang, F. Chen, Z. Li, G. Shen, D. Cui and X. Chen, Adv. Mater., 2012, 24, 5104-5110.

365 D. W. Zheng, B. Li, C. X. Li, J. X. Fan, Q. Lei, C. Li, Z. Xu and X. Z. Zhang, ACS Nano, 2016, 10, 8715-8722.

366 F. Wu, L. Yue, H. Su, K. Wang, L. Yang and X. Zhu, Nanoscale Res. Lett., 2018, 13, DOI: 10.1186/s11671-0182761-5.
367 Y. Wen, Q. Jia, F. Nan, X. Zheng and W. Liu, Chem.-Asian J., 2019, 14, 2162-2168.

368 H. Fan, X. Yu, K. Wang, Y. Yin, Y. Tang, Y. Tang and X. Liang, Eur. J. Med. Chem., 2019, 182, 111620.

369 J. D. Monroe, E. Belekov, A. O. Er and M. E. Smith, Photochem. Photobiol., 2019, 95(6), 1473-1481.

370 X. Bao, Y. Yuan, J. Chen, B. Zhang, D. Li, D. Zhou, P. Jing, G. Xu, Y. Wang, K. Holá, D. Shen, C. Wu, L. Song, C. Liu, R. Zbořil and S. Qu, Light: Sci. Appl., 2018, 7, 1-11.

371 B. Geng, D. Yang, D. Pan, L. Wang, F. Zheng, W. Shen, C. Zhang and X. Li, Carbon, 2018, 134, 153-162.

372 M. Thakur, M. K. Kumawat and R. Srivastava, RSC Adv., 2017, 7, 5251-5261.

373 Z. Tian, X. Yao, K. Ma, X. Niu, J. Grothe, Q. Xu, L. Liu, S. Kaskel and Y. Zhu, ACS Omega, 2017, 2, 1249-1258.

374 H. Liu, C. Li, Y. Qian, L. Hu, J. Fang, W. Tong, R. Nie, Q. Chen and H. Wang, Biomaterials, 2020, 232, DOI: 10.1016/j.biomaterials.2019.119700.

375 S. Zheng, Z. Jin, C. Han, J. Li, H. Xu, S. Park, J. O. Park, E. Choi and K. Xu, J. Mater. Sci., 2020, 55, 1184-1197.

376 Y. Gao, S. Zhong, L. Xu, S. He, Y. Dou, S. Zhao, P. Chen and X. Cui, Microporous Mesoporous Mater., 2019, 278, 130-137.

377 M. Lan, L. Guo, S. Zhao, Z. Zhang, Q. Jia, L. Yan, J. Xia, H. Zhang, P. Wang and W. Zhang, Adv. Ther., 2018, 1, DOI: 10.1002 /adtp.201800077.

378 F. Wo, R. Xu, Y. Shao, Z. Zhang, M. Chu, D. Shi and S. Liu, Theranostics, 2016, 6, 485-500.

379 Y. Xuan, R. Zhang, D. Zhao, X. Zhang, J. An and K. Cheng, Chem. Eng. J., 2019, 369, 87-99.

380 S. Badrigilan, B. Shaabani, N. Gharehaghaji and A. Mesbahi, Photodiagn. Photodyn. Ther., 2019, 25, 504-514. 